\title{
A Code-Division, Multiple Beam Sonar Imaging System
}

by

\author{
John Michael Richardson \\ B.S., United States Naval Academy (1982) \\ Submitted in partial fulfillment of the \\ requirements for the degree of \\ ELECTRICAL ENGINEER \\ and \\ MASTER OF SCIENCE IN ELECTRICAL ENGINEERING \\ at the \\ MASSACHUSETTS INSTITUTE OF TECHNOLOGY \\ and the \\ WOODS HOLE OCEANOGRAPHIC INSTITUTION
}

August 1989

(c) John Michael Richardson, 1989

The author hereby grants to MIT and WHOI permission to reproduce and

\begin{tabular}{|c|}
\hline MARINE \\
BIOLOGICAL \\
LABORATORY \\
\hline LIBRARY \\
\hline $\begin{array}{c}\text { WOODS HOLE, MSS. } \\
\text { W. H. O. I. }\end{array}$ \\
\hline
\end{tabular}
to distribute copies of this thesis document in whole or in part.

Signature of

Author....

Joint Program in Electrical Engineering Massachusetts Institute of Technology Woods Hole Oceanographic Institution

Certified August 11. 1989

by

Dr. Timothy K. Stanton Woods Hole Oceanographic Institution

Certified Thesis Supervisor

by

Dr. Áán V. Oppenheim Massachusetts Institute of Technology

Accepted Thesis Sunerfisar

by

Dr. W. Kendall Melville

Chairman, Joint Committee for Oceanographic Engineering Massachusetts Institute of Technology/Woods Hole Oçeanographic Institution

Accepted

by

Dr. Arthur Smith

Chairman, Electrical Engineering Dept. on Graduate Students Massachusetts Institute of Technology

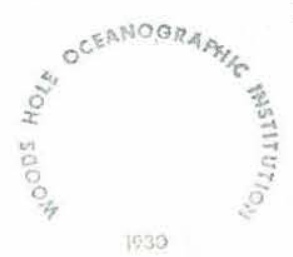




\title{
A Code-Division, Multiple Beam Sonar Imaging System
}

\author{
by \\ John Michael Richardson \\ Submitted to the Department of Electrical Engineering \\ on August 11, 1989 in partial fulfillment of the requirements \\ for the MIT/WHOI Joint Program degrees of Electrical Engineer and \\ Master of Science in Electrical Engineering
}

\begin{abstract}
In this thesis, a new active sonar imaging concept is explored using the principle of code-division and the simultaneous transmission of multiple coded signals. The signals are sixteen symbol, four-bit, non-linear, block Frequency-Shift Keyed (FSK) codes, each of which is projected into a different direction. Upon reception of the reflected waveform, each signal is separately detected and the results are inverted to yield an estimation of the spatial location of an object in three dimensions. The code-division sonar is particularly effective operating in situations where the phase of the transmitted signal is perturbed by the propagation media and the target. Most imaging techniques presently used rely on preservation of the phase of the received signal over the dimension of the receiving array. In the code-division sonar, spatial resolution is obtained by using the combined effects of code-to-code rejection and the a-priori knowledge of which direction each code was transmitted. The coded signals are shown to be highly tolerable of phase distortion over the duration of the transmission. The result is a highresolution, three-dimensional image, obtainable in a highly perturbative environment. Additionally, the code-division sonar is capable of a high frame rate due to the simplicity of the processing required. Two algorithms are presented which estimate the spatial coordinates of an object in the ensonified aperture of the system, and the performance of the two is compared for different signal to noise levels. Finally, the concept of codedivision imaging is employed in a series of experiments in which a code-division sonar was used to image objects under a variety of conditions. The results of the experiments are presented, showing the resolution capabilities of the system.
\end{abstract}

Thesis Supervisors:

Dr. Alan Oppenheim

Massachusetts Institute of Technology

Dr. Tim Stanton

Woods Hole Oceanographic Institution 


\section{Acknowledgements}

One can not hope to succeed in a graduate program such as the MIT/WHOI Joint Program without heavily depending on the generous help of many people. In my case, I know that without this assistance, I would not have been able to accomplish anything close to the thesis that follows. I am particularly grateful to the Ocean Engineering Technicians in the Smith Building at Wood's Hole Oceanographic Institution and at the Marine Physical Lab at Scripps Institution of Oceanography for helping me through constructing the prototype code-division sonar and performing the experiments. I am thankful to Dr. Josko Catipovic for the construction of the control circuit for the sonar. Dr. Arthur Baggeroer was invaluable for his fabulous teaching and his administrative advice throughout this project. Thanks to Dr. Timothy Stanton for his help on the completion of this thesis.

I am especially grateful to Dr. Alan Oppenheim, who paid me the distinct honor of advising this thesis, and allowed me to be part of the most stimulating research group at MIT. Al, I hope the summer winds at WHOI always push the upper end of the Beaufort scale.

Finally, there is Dr. Jules Jaffe. It was his creativity that conceived the code-division idea, and his positive inspiration that kept this thesis on track. Working with him has given me the unique experience of being near a real 'idea man.' For Jules, there is no concept that is out of bounds, no problem that doesn't present new opportunities. For me, the best part of this program was getting to know Jules and learn under the influence of his supervision.

The real value of a program like this is the chance to work with the brightest people. It is them that I will remember long after the theories have grown dim. 


\section{Contents}

1 Introduction 9

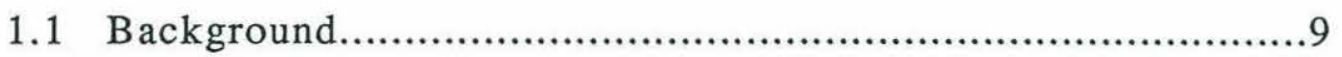

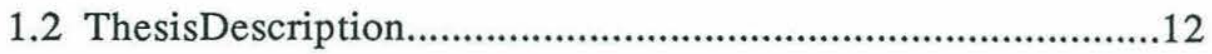

2 Time/Space Processes and Active Sonar Principles 14

2.1 Representing Samples of a Time/Space Process.................................14

2.2 Applications to Active Sonar Imaging............................................17

3 Signal Design and Representation 20

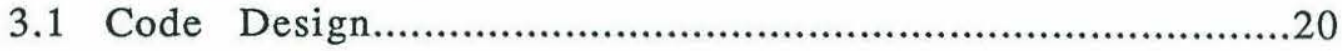

3.2 Signal Construction and Representation...........................................23

3.3 Signal Performance in an Imaging Scenario.....................................28

$4 \quad$ Receiver Design $\quad 36$

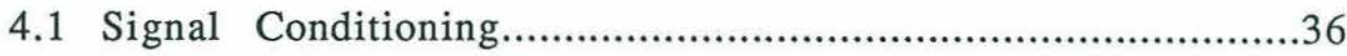

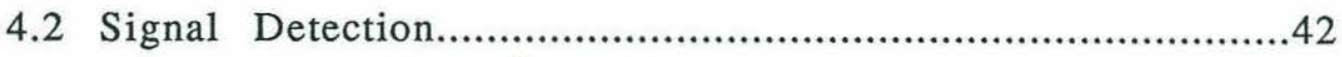

4.2.1 Design of the Signal Detector................................................42

4.2.2 Further Developments in Signal Design....................................47

4.3 Spatial Energy Distribution of the Signals.........................................49

4.4 Signal Parameter Estimation.......................................................54

5 Target Data Estimation $\quad 60$

5.1 Spatial Correlation of the Signals Inside the Ensonified Aperture.............60

5.2 Constrained Method for Location Estimation......................................63

5.3 Minimum Mean-Square Difference Estimator....................................67

6 Experimantal Procedure and Results $\quad 70$

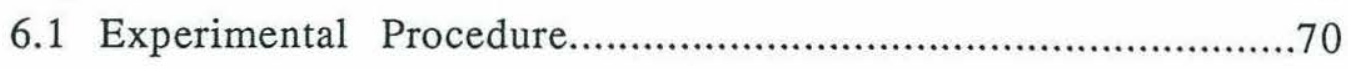




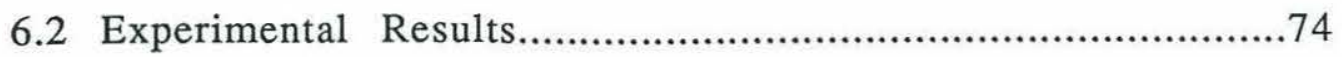

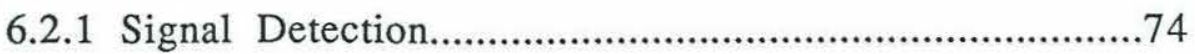

6.2.2 Target Location and Image Construction....................................77

7 Conclusions $\quad 82$

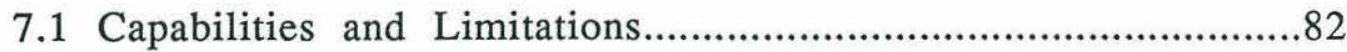

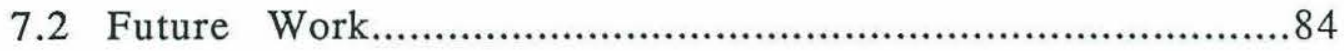

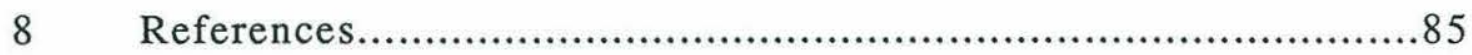




\section{List of Figures}

2-1 Forming the Observation Space and Characterizing a Space/Time Process..........15

3-1 Wheel of Fortune for Generating Frequency Hop Codes of length $q=17 \ldots \ldots \ldots . .21$

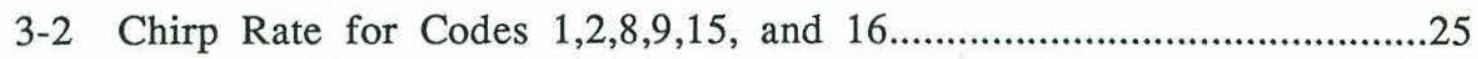

3-3 Auto-Ambiguity Functions for Signals Eight (a), and Twelve (b)....................31

3-4 Contours of the Auto Ambiguity Functions for Signals 1, 2, 3, and 4..............33

3-5 Cross Ambiguity Functions for Signals One/Two (a), and Four/Eight (b)...........34

3-6 Contours of the Cross-Ambiguity Functions for Signals Two/Three (a) and

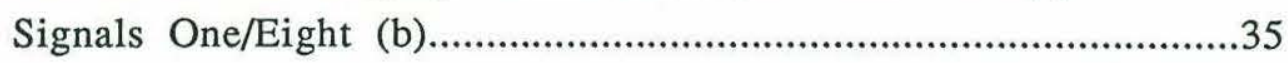

4-1 Steps Taken to Pre-condition the Received Signal.......................................37

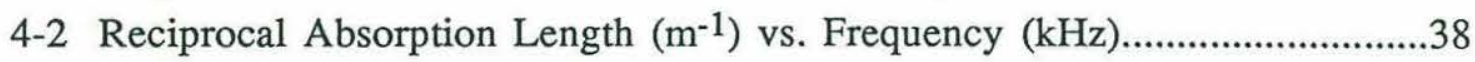

4-3 Attenuation of Signal Energy by the Medium (at $900 \mathrm{kHz}, 5^{\circ} \mathrm{C}$ )....................39

4-4 Power Spectral Density Function of Received Signals Before (a), and After (b) Filtering.................................................................41

4-5 Theoretical Coherent Detection for Signal Eight........................................44

4-6 Probability of Decision Error for the Coherent and Incoherent Receivers

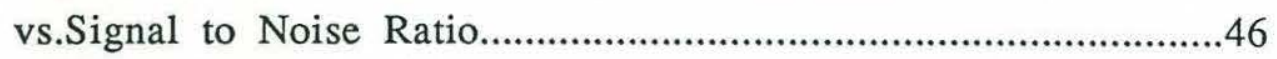

4-7 Correlation Sidelobes for Three Windows...............................................47

4-8 Coordinate System Convention Inside Aperture.........................................51

4-9 Individual Illumination for Beam One (a) and all Sixteen Beams (b)

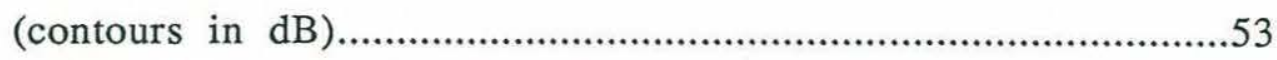

4-10 Distribution of $\beta$ as Receiver SNR Increases........................................58

5-1 Spatial Correlation Functions for Illumination Coefficients 1,2 (a) and for Coefficients 1,16 (b). 
5-2 Spatial Response of the Constrained Estimator

(target at the center of the aperture).

5-3 Comparison of the Resolution Capability of the Conventional Beam

(dashed line) and the Constrained Estimator (solid line).

5-4 Performance of the Minimum Mean-Square Difference Estimator

for $\sigma=10 \mathrm{~dB}(\mathrm{a}), 3 \mathrm{~dB}(\mathrm{~b}), 0 \mathrm{~dB}$ (c) and $-3 \mathrm{~dB}(\mathrm{~d})$.

6-1 Effective Narrowband Jammer Created by Close Proximity of Tank Bottom......71

6-2 Configuration for Imaging Experiments..............................................72

6-3 Detection of Target in Beam Two Coherent (a) and Incoherent (b).(Phase One)...75

6-4 Coherent Detection of a Single Target in Beam 16 (Phase Two).

6-5 Coherent Detection of Two Targets, one each in Beam 11 and 15 (Phase Three)..76

6-6 Coherent Detection of a Target in Beam 8 Imaged Through a Fluctuating

Medium (Phase Four)

6-7 Detection of Target in Beam Six, (a) Constrained Estimator,

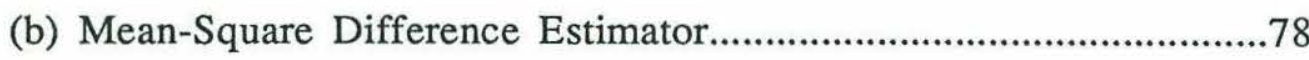

6-8 Target Detection in Lower-Right Corner of the Aperture (a) Constrained Estimate

and (b) Mean-Square Difference Estimate

.79

6-9 Improved Resolution Obtained as Target Moves into Aperture (a) Constrained

and (b) Mean-Square Difference.

6-10 Detection of Range Separated Targets (a) Constrained

and (b) Mean-square Difference 


\section{Chapter 1}

\section{Introduction}

\subsection{Background}

As the world pace speeds up, the need to understand the environment beyond one's immediate field of view is crucial to making correct decisions. Detection of things at distances where it is impossible to use the human sensory system has motivated the development of sophisticated remote sensing devices. These sensors can transmit large amounts of information from great distances to a central location where it can be assessed by the operator.

There are a wide variety of ways to obtain images from a remote location. The current generation of sensors employ electromagnetic radiation in the form of light for visual systems, radar waves, and x-rays and magnetic fields for medical purposes. The best choice of imaging technique is often dictated by the imaging environment. One good example of this is underwater imaging in the ocean. Here, the properties of the water make it impossible to use visual or radar techniques due to the high attenuation of electromagnetic radiation in the ocean. In the ocean environment, sonar is the primary means for remote sensing. These can be broadly classified into two groups: passive sonars, which merely receive the existing sound field; and active sonars, which transmit a signal and receive the reflection of this signal from distant objects. 
Within these two broad categories, sonars can vary widely in complexity. One can describe the complexity of an imaging sonar in terms of how well it is able locate objects in three-dimensional space. The most simple sonars are one-dimensional sonars. An example of a one-dimensional active sonar is the fathometer. This sonar sends out a single burst of sound from the sea surface, and waits for the reflection from the bottom. The time-delay between transmission and reception yield the depth of the water, a one-dimensional quantity. A side-scan sonar increases the complexity to two dimensions. A side-scan sonar transmits a thin beam that images a "slice" of the bottom. By piecing the slices together properly, a picture of the bottom may be obtained. With these images, there is ambiguity concerning the depth vs. bottom location of the objects in the image, because the sonar is only able to generate a two-dimensional location. Finally, there are full three-dimensional imaging sonars that can obtain both the direction and range to an object. These systems typically employ large, multi-element arrays, or a single steered narrow beam to obtain the position of the object.

The introduction of digital processing techniques initiated a renaissance in highresolution sonar imaging. The Fast Fourier Transform [1] coupled with high speed computers has made it possible to develop previously inconceivable systems that operate in real time. Additionally, there have been developments in new theories for high-resolution beamformers such as the Maximum Liklihood Method [2], Maximum Entropy Method [3], eigenvector methods [4, 5], and matched-field processing [6]. These advances have made true high-resolution underwater imaging possible at distances well beyond the maximum range for light or radar.

While highly successful, there are some drawbacks to these methods. The high resolution methods mentioned above all require large, multi-element arrays. Additionally, some coherence of the received signal across the dimension of the array is required. In some applications, these restrictions can result in poor performance. The single, steered beam is simpler in theory than the multi-element arrays, but this sensor 
may not detect transient occurrences outside the beam when it is steered to a different part of the aperture.

This thesis explores a new method for obtaining three-dimensional underwater images that can offer both a high frame rate and high resolution in a phase-perturbative environment. First introduced by Jaffe et. al. [7, 8], it is based on the principle of spatially,variant insonification, where multiple coded signals are simultaneously projected into different directions in space. Each signal will reflect from the objects that are located within their beam pattern. The total reflected signal is composed of a superposition of these individual reflections. The codes used in the system are frequency-hop codes, allowing one to separately detect the individual coded signals from the total reflected. This information, coupled with the a-priori knowledge of where each signal was projected, make it possible to invert the information present in the received signal to a three-dimensional representation of the objects that were ensonified by the transmitted energy. The concepts employed in this system are adapted from information and communication theory, and applied to the problem of underwater imaging to more fully exploit the information capacity of the underwater acoustic channel.

In order to employ such a system, a set $\{\mathrm{S}\}$ of coded signals is needed. As is typical, the range resolution of the system depends on the width of the autocorrelation function of each coded signal in the set. In the spatially-variant insonification sonar, the directional resolution depends on the cross-correlation of each signal to every other signal in the set. Ideally, each signal should have an impulsive autocorrelation function and a zero cross-correlation function with all other signals. A signal design strategy would then be to construct the set $\{\mathrm{S}\}$ such that:

$$
\min _{\{\mathrm{S}\}} \int_{-\infty}^{\infty} \mathrm{s}_{\mathrm{i}}(\mathrm{t}) \mathrm{s}_{\mathrm{j}}(\mathrm{t}-\tau) \mathrm{dt} \quad \mathrm{i} \neq \mathrm{j}, \forall \tau
$$

and that: 


$$
\int_{-\infty}^{\infty} s_{i}(t) s_{i}(t-\tau) d t=\delta(\tau)
$$

for all $s_{i}(t), s_{j}(t)$ in the set $\{S\}$.

A set of codes having these properties was proposed in the original references $[7,8]$ in an multi-beam active sonar context. Although conceived, the system proposed by Jaffe, et. al. was never implemented or tested experimentally. Additonally, no consideration was given to the means by which an image can be constructed from the reflected waveform. It is these questions that are the principle topics of this thesis. In particular, the following questions are addressed:

1. What is the best way to implement the frequency-hop codes presented in $[7,8]$ as transmitted waveforms?

2. Are the codes suitable for sonar imaging?

3. What steps are necessary to detect the individual codes, and once detected, how can an ensonified target be located in space?

4. Is a system that employs spatially-variant insonification realizable?

These answers to these questions are critical to assessing the true value of this new imaging technique. This thesis presents two methods for the construction of threedimensional images. In order to fully verify these methods, a multi-beam sonar was constructed and used in a series of experiments. The major conclusion of the thesis will be to demonstrate the suitability of the code-division, multiple beam sonar imaging.

\subsection{Thesis Description}

Chapter Two serves primarily as a basic review, and discusses the fundamentals of processing stochastic signals. Chapters Three, Four, and Five explore the theory involved with processing the coded signals to achieve a representation for the location of 
an ensonified object. Chapter Three is a detailed discussion of the code design, and introduces the mathematical representation for the signals. This chapter also examines the theoretical suitability of the signals for an imaging scenario. In Chapter Four, the receiver for the coded signals is designed. Pre-conditioning the received signal is described, and the decision rules used to detect an individual coded signal are found. The chapter ends with the development of a method to estimate signal parameters needed to determine the spatial location of an object in the ensonified field. Chapter Five presents two methods to estimate the location of an object placed in the field of view of the sonar. The two methods are compared in terms of resolution and stability under varying noise levels. Chapter Six describes a series of experiments that were performed with a codedivision, multiple beam sonar that was constructed to test the system in a variety of underwater imaging tasks. The results of the experiments are also presented in this chapter. The final chapter provides a brief summary of the capabilities and limitations of the code-division imaging technique. 


\section{Chapter 2:}

\section{Time/Space Processes and}

\section{Active Sonar Applications}

Active acoustic imaging involves generating a known signal, and sampling the spatially and temporally varying field created by the reflection of that signal. Once this is done, the next task is converting the samples into a representation of the information contained in that field. The methods by which this is done are varied; some of them were mentioned in the previous chapter. This chapter reviews some fundamental issues associated with sampling a space/time stochastic process. Although the concepts presented are well-known, they serve as the foundation for the more complex developments later in the thesis.

\subsection{Representing Samples of a Space/Time Process}

A space/time random process can be thought of as the sample space of a four-dimensional process that is characterized by an unknown probability distribution. Naturally, it is impossible to completely know the process; only portions of the process

are obtained through sampling. These samples form the observation space, which is a 
subset of the sample space. As such, an underwater acoustic field is a four-dimensional space/time process. If there are several point receivers forming an array, each sampling the acoustic field in time, then the observation space is a collection of time series, one from each of the receivers (see Figure 2-1).

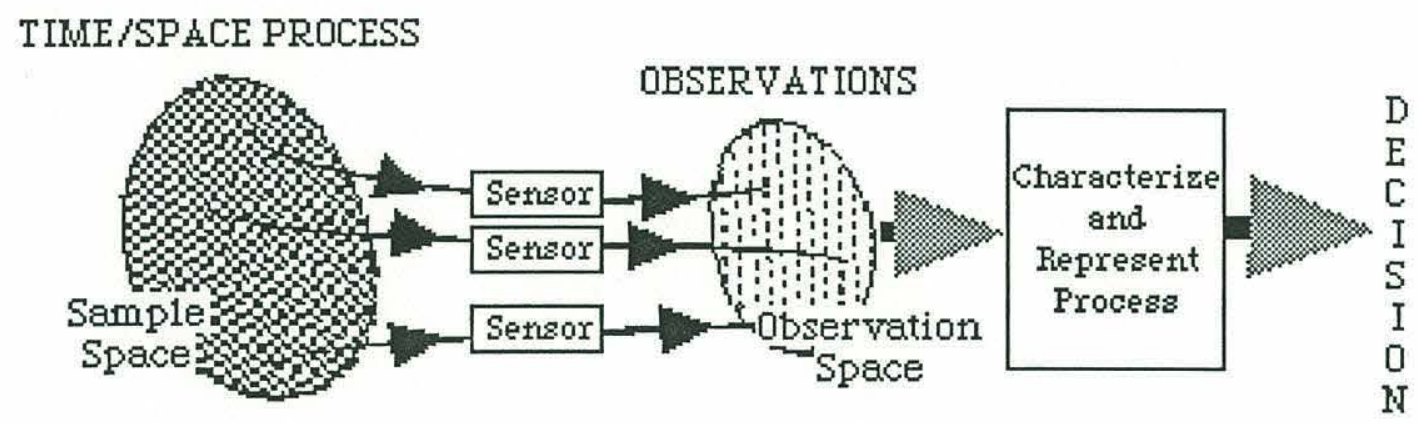

Figure 2-1: Forming the Observation Space and Characterizing a Space/Time process

From the observation space, it is generally possible to make at least a second-moment characterization of the sampled space/time process. Obviously, the first moment characterization is the mean of the process:

$$
\mathrm{m}_{\mathrm{x}}(\mathrm{t} ; \mathbf{z})=\mathrm{E}\{\mathrm{x}(\mathrm{t} ; \underline{\mathbf{z}})\}
$$

Once the mean is found, an equivalent zero-mean process that is formed by subtraction. This equivalent, zero-mean process will be assumed henceforth. The second-order characterization of the process is the space/time covariance function, $\mathrm{R}_{\mathrm{x}}\left(\mathrm{t}_{1}, \mathrm{t}_{2} ; \mathbf{z}_{1}, \underline{\mathbf{Z}}_{2}\right)$ :

$$
\begin{aligned}
& \mathrm{R}_{\mathrm{x}}\left(\mathrm{t}_{1}, \mathrm{t}_{2} ; \mathrm{z}_{1}, \mathrm{z}_{2}\right)=\mathrm{E}\left\{\left(\mathrm{x}\left(\mathrm{t}_{1} ; \mathrm{z}_{1}\right)-\mathrm{m}_{\mathrm{x}}\left(\mathrm{t}_{1} ; \mathrm{z}_{1}\right)\right)\left(\mathrm{x}\left(\mathrm{t}_{2} ; \mathrm{z}_{2}\right)-\mathrm{m}_{\mathrm{x}}\left(\mathrm{t}_{2} ; \mathrm{z}_{2}\right)\right)^{*}\right\} \\
& =\mathrm{E}\left(\mathrm{x}\left(\mathrm{t}_{1} ; \underline{\mathrm{z}}_{1}\right) \mathrm{x}^{*}\left(\mathrm{t}_{2} ; \mathrm{z}_{2}\right)\right) \quad \text { for a zero-mean process. }
\end{aligned}
$$

By making some standard assumptions about the space/time covariance function, it is possible to arrive at more useful representations of the process. Many situations en- 
countered can be considered wide-sense stationary over the processing interval, thereby reducing (2.1.1) to the form:

$$
\mathrm{R}_{\mathrm{x}}\left(\mathrm{t}_{1}, \mathrm{t}_{2} ; \mathbf{z}_{1}, \underline{\mathbf{z}}_{2}\right) \Rightarrow \mathrm{R}_{\mathrm{x}}\left(\tau ; \mathbf{z}_{1}, \underline{\mathbf{z}}_{2}\right) \quad \text { where } \tau=\mathrm{t}_{2}-\mathrm{t}_{1} \text {. }
$$

The assumption of spatial homogeneity over the receiving aperture, further reduces the space/time covariance function:

$$
\mathrm{R}_{\mathrm{x}}\left(\tau ; \underline{\mathbf{z}}_{1}, \underline{\mathbf{z}}_{2}\right) \Rightarrow \mathrm{R}_{\mathrm{x}}(\tau ; \xi) \quad \text { where } \xi=\mathbf{z}_{2}-\mathbf{z}_{1} \text {. }
$$

From this model, three spectral estimate of the process can be found. The first is the temporal spectrum, which performs a spectral decomposition in time:

$$
S_{x}(\omega ; \xi)=\int_{-\infty}^{\infty} R_{x}(\tau ; \xi) e^{-j \omega \tau} d \tau
$$

The next is the spatial wavenumber spectrum:

$$
F_{x}(\tau ; \underline{k})=\iint_{-\infty}^{\infty} \int_{x} R_{x}(\tau ; \xi) e^{j(\underline{k} \xi)} d \xi
$$

where the integration takes place over the spatial dimensions of the process. The third and mostly useful decomposition is the frequency/wavenumber function, which is a spectral decomposition in both time and space:

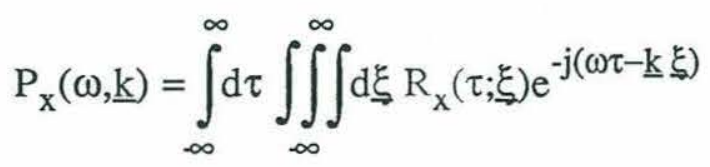

If the product of the frequency/wavenumber functions for two processes is zero, then the processes are independent and uncorrelated. The frequency/wavenumber function 
has the effect of decomposing any process into a sum of plane waves with frequency $\omega$ and wavevector $\xi$. Each plane wave is an impulse in frequency/wavenumber space.

So far, this treatment hold true for any space/time random process. If the process is restricted to satisfy the wave equation, then the frequency and wavenumber are coupled by the dispersion relation, $|\mathrm{k}|=\omega / \mathrm{c}$ ( $\mathrm{c}$ being the velocity of propagation in the medium). By imposing this constraint, the frequency/wavenumber space need only be examined on the sphere where $|\mathrm{k}|=\omega / \mathrm{c}$.

This analysis offers tremendous flexibility in modelling space time processes that may occur in the real ocean environment. Any process which can be represented as a superposition of plane waves fits immediately into the framework presented here. Many examples of different applications can be found in [9], which develops this framework more completely and applies it to a wide variety of processes. A particular application of interest is the definition of a spatial response function for a sonar system aperture. This is more fully developed in the following section.

\subsection{Aperture Response to a Time/Space Process}

The sonar aperture is a time/space filter through which the acoustic time/space process is passed. For the purposes of this treatment, it will be assumed that the filter is time-invariant at each location on the aperture, so that the time/space weighting function can be expressed as:

$$
\mathrm{g}\left(\mathrm{t}_{1}, \mathrm{t}_{2} ; \underline{\mathrm{z}}\right)=\mathrm{g}\left(\left(\mathrm{t}_{1}-\mathrm{t}_{2}\right) ; \underline{\mathrm{z}}\right)=\mathrm{g}(\tau ; \underline{\mathrm{z}})
$$

From (2.1.3) and (2.1.5), the spectral decompositions of the aperture weighting function follow easily. The frequency/space correlation function is: 


$$
G(\omega ; \underline{z})=\int_{-\infty}^{\infty} g(\tau ; \underline{z}) e^{-j \omega \tau} d \tau
$$

and the frequency/wavenumber response is:

$$
\mathfrak{I}(\omega ; \underline{\mathrm{k}})=\int_{\Omega} \mathrm{G}(\omega ; \underline{\mathbf{z}}) \mathrm{e}^{\mathrm{j} \underline{\mathrm{k}} \cdot \mathbf{z}} \mathrm{d} \mathbf{z}=\iint_{\Omega^{-\infty}}^{\infty} \mathrm{g}(\tau ; \underline{\mathbf{z}}) \mathrm{e}^{-\mathrm{j}(\omega \tau-\underline{\mathrm{k}} \cdot \mathbf{z})} \mathrm{d} \tau \mathrm{d} \underline{\mathbf{z}}
$$

where $\Omega$ defines the extent of the array. This function describes the response of the aperture with a time/space transfer function $\mathrm{g}(\tau ; \mathbf{z})$ to a plane wave with frequency $\omega$ and wavevector $\underline{\mathrm{k}}$. Typically, in applying this function to a real process, the frequency $\omega$ is fixed, and the spatial dependence of the process is examined. The primary aperture of interest for this system is the uniformly-weighted circular disc, which is used to transmit the coded waveforms:

$$
g(\tau ; r)=\left\{\begin{array}{cc}
\frac{1}{2 \pi R_{\Omega}^{2}} & r \leq R_{\Omega} \\
0 & r \geq R_{\Omega}
\end{array}\right\}
$$

The frequency/wavenumber response of this aperture can be found directly:

$$
\begin{aligned}
& \mathfrak{I}(\omega ; \underline{\mathrm{k}})=\int_{0}^{\mathrm{R}_{\Omega}} \mathrm{r} \mathrm{dr} \int_{0}^{2 \pi} \mathrm{d} \theta\left(\frac{1}{2 \pi \mathrm{R}_{\Omega}^{2}}\right) \mathrm{e}^{\mathrm{j}\left[\left(\mathrm{k}-\mathrm{k}_{0}\right) \cdot \mathrm{r}\left(\underline{a}_{\mathrm{x}} \cos \theta+\underline{\mathrm{a}}_{\mathrm{y}} \sin \theta\right)\right]} \\
& =\frac{\mathrm{J}_{1}\left(\mathrm{R}_{\Omega} \sqrt{\left(\mathrm{k}-\mathrm{k}_{0}\right)_{\mathrm{x}}^{2}+\left(\mathrm{k}-\mathrm{k}_{0}\right)_{\mathrm{y}}^{2}}\right)}{\mathrm{R}_{\Omega} \sqrt{\left(\mathrm{k}-\mathrm{k}_{0}\right)_{\mathrm{x}}^{2}+\left(\mathrm{k}-\mathrm{k}_{0}\right)_{\mathrm{y}}^{2}}}
\end{aligned}
$$

where $\underline{\mathrm{k}}_{\mathrm{o}}$ is the wavevector associated with a transmitted plane wave and $\left(\mathrm{k}-\mathrm{k}_{\mathrm{o}}\right)_{\mathrm{x}}$ is the difference in the $\mathrm{x}$-projections of the two wavevectors $\underline{\mathrm{k}}$ and $\underline{\mathrm{k}}_{\mathrm{o}}$ (likewise with $\left.\left(\mathrm{k}-\mathrm{k}_{\mathrm{o}}\right)_{\mathrm{y}}\right)$. 
For this specific sonar, the transmitted signals have wavevectors perpendicular to the aperture. In this instance, the aperture response is:

$$
\mathfrak{I}(\omega ; \underline{\mathrm{k}})=\left(\frac{\mathrm{J}_{1}\left(\mathrm{kR}_{\Omega} \sin \alpha\right)}{\mathrm{kR}_{\Omega} \sin \alpha}\right)
$$

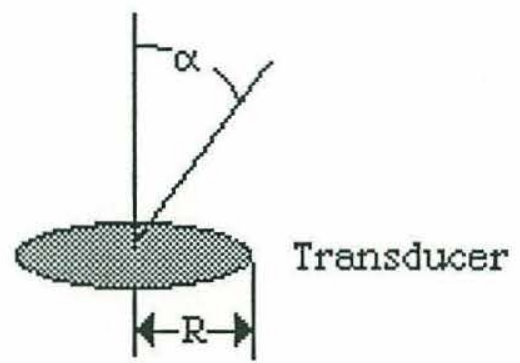

where $\mathrm{k}=2 \pi / \lambda$. The square of the response function yields the standard beam pattern for the circular disc aperture.

While this is a simplistic case, it is important to keep the broader idea of the beam pattern being a specific application of the frequency/wavenumber response function. In Chapter Five, response functions will be defined for the code-division sonar which will expand on the concepts presented here. 


\section{Chapter 3}

\section{Signal Design and Representation}

\subsection{Code Design}

The codes used in the multi-beam system are non-binary, non-linear FrequencyShift Keyed (FSK) codes that are developed from a $q$-ary alphabet where $q$ is a prime number. Given $q$, the permutation operator will construct a set of $q-1$ codes, all of which nearly have the properties stated in (1.1) and (1.2).

The construction and properties of the codes are fully described in the original references $[7,8]$. A brief description is provided here only as it directly relates to the generation of the set of codes to be used. Given the prime number $q$, there will be a total of $q-1$ code words constructed. The $\mathrm{n}$ chips of the mth code word are ordered according to the formula:

$$
\mathrm{C}_{\mathrm{m}}=\left\{\mathrm{c}_{\mathrm{m}, \mathrm{n}}\right\}=|\{\mathrm{m}+\mathrm{nm}\}|_{\text {modulo } q} \quad \begin{aligned}
& \mathrm{m}=1,2, \ldots, q-1 \\
& \mathrm{n}=0,1,2, \ldots, q-1
\end{aligned}
$$

where $\mathrm{C}_{\mathrm{m}}$ is the entire code word, and $\mathrm{c}_{\mathrm{m}, \mathrm{n}}$ are the chips that comprise the code word. This was described as the "wheel of fortune" operator, due to the cyclic nature of the code generation (see Figure 3-1). The first code word is generated by starting on the first element, and counting around the wheel by ones until all the alphabet has been used once. The next code word is generated by starting at two, and counting by twos until all 
the alphabet has been used. The third word begins on the number three and counts by threes, and so forth, until this has been done for $q-1$ words.

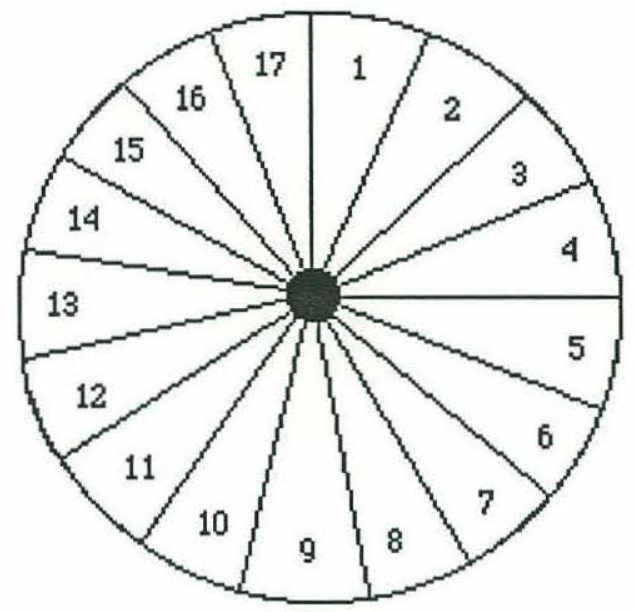

Figure 3-1: Wheel of Fortune for Generating Frequency Hop Codes of length $q=17$.

When this generating process is applied to an alphabet of seventeen, the set of code words that result is:

$$
\begin{aligned}
& C_{1}=\{1234567891011121314151617\}=C_{-16} \\
& C_{2}=\{2468101214161357911131517\}=C_{-15} \\
& C_{3}=\{3691215147101316258111417\}=C_{-14} \\
& C_{4}=\{4812163711152610141591317\}=C_{-13} \\
& C_{5}=\{5101538131611164914271217\}=C_{-12} \\
& C_{6}=\{6121713281439154101651117\}=C_{-11} \\
& C_{7}=\{7144111815512291661331017\}=C_{-10} \\
& C_{8}=\{8167156145134123112101917\}=C_{-} 9
\end{aligned}
$$

where the notation $\mathrm{C}_{-\mathrm{i}}$ is to indicate that the order of the chips is reversed, thus $\mathrm{C}_{16}$ is $\mathrm{C}_{1}$ in reverse order. A property of this set of codes is that a given code word possesses all 
its original properties (including those stated in (1.1) and (1.2)) under all cyclic shifts of the elements in any code word in the set. For example, if

$$
\begin{aligned}
& C_{1}=\{1234567891011121314151617\} \quad \text { is a code word, then } \\
& C_{1}{ }^{\prime}=\{1712345678910111213141516\}
\end{aligned}
$$

is a code word that can replace (but not exist with) the original $C_{1}$. It is apparent now that given the above set, an equivalent set of codes could be constructed in which every code begins with the element seventeen. This 'lead chip' can be used by the receiver as a flag to indicate that a code word has arrived. This knowledge can offer a tremendous savings in computational effort for the rest of the code. When each element of the code alphabet is assigned to a distinct frequency, the set of codes can be reinterpreted as a set of 16-chip frequency hop codes, each code being preceded by a common flag (chip 17), which offers no information about code-to-code discrimination. When viewed this way, the codes can be classified as sixteen symbol, four bit (expressed by the notation $(16,4))$ FSK block codes very similar to Reed-Solomon codes [10]. The code rate is 0.25 bits per symbol. The Hamming Distance of the set is defined as the number of elements that differ between any two code words. For this set, the Hamming Distance is sixteen, and the codes can be guaranteed correct up to seven symbol errors. Additionally, as required by the property stated in (1.1), this large distance is maintained over all cyclic shifts of any two codes: there is at most only one matching element between any two code words for all possible shifts between any two codes in this set.

Generally, given an alphabet of $\mathrm{M}=(q-1)$ elements, it is possible to generate $\mathrm{M}$ ! different words of length $M$. While this operator generates $M$ « $M$ ! codes, it is guaranteed that the set will always have this large distance property that makes them so suitable for imaging without employing a separate synchronization scheme. In this situation, the desire to maintain the code-word separability under all time shifts justifies the inefficient use of the available bandwidth. 


\subsection{Signal Construction and Representation}

The actual signals that are transmitted follow directly from the code words.

Given a code word, $\mathrm{C}_{\mathrm{i}}$, the signal $\mathrm{S}_{\mathrm{i}}$, can be generated by assigning a frequency chip to each element of the $q$-ary alphabet. The order in which the elements are arranged in a given code dictates the order in which the frequencies are transmitted. If each chip is of duration $\mathrm{T}$, then in order to ensure minimum cross-correlation, the frequency spacing, $\Delta$, must obey:

$$
\Delta \mathrm{f}=\frac{\mathrm{m}}{\mathrm{T}} \quad \mathrm{m}=1,2,3, \ldots
$$

If $\Delta \mathrm{f}$ is chosen to be $1 / \mathrm{T}$, the total signal bandwidth is then:

$$
\mathrm{W}=\mathrm{M} \Delta \mathrm{f}=\frac{\mathrm{M}}{\mathrm{T}}
$$

which results in a time-bandwidth product of:

$$
\mathrm{WMT}=\left[\frac{\mathrm{M}}{\mathrm{T}}\right] \mathrm{MT}=\mathrm{M}^{2}
$$

This directly relates the maximum size of the signal set to the information capacity of the underwater acoustic channel. The time-bandwidth product of the frequency-hop codes is typically a factor of $\mathrm{M}$ higher than a comparable length binary code. For the M-length binary code, the chip duration $T$ will be the same, and the bandwidth will be only $1 / T$, resulting in a total time-bandwidth product of $\mathrm{M}$. For a limited channel capacity, it would be possible to fit a factor of $\mathrm{M}$ more binary codes in the same channel. The relatively inefficient use of the available channel capacity by the frequency-hop codes is offset in a code-division imaging application because the code rejection requirements set 
forth in (1.1) are more strict than a typical communication channel, where a more efficient coding scheme might be employed. In the underwater acoustic channel, the capacity of the channel is typically far greater than either $\mathrm{M}$ or $\mathrm{M}^{2}$, and this issue is not important. As long as the signals are constructed as outlined above, the set will have the desired signal-to-signal rejection properties. For the specific signal used in this thesis, the lowest frequency $(700 \mathrm{kHz})$ will be assigned to element 1 of the alphabet, and the highest frequency $(1100 \mathrm{kHz})$ will be assigned to element 17 of the alphabet. The frequency separation will be $25 \mathrm{kHz}$ between each chip. Examination of the signals reveals that they exhibit a great deal of structure in the time-frequency domain, resembling discrete samples of a continuous chirp signal. As is evident by the list of code words, the details of the signal structure can be completely defined by the first eight code words since the last eight are duplicates of the first eight, transmitted in reverse order. The first signal, $\mathrm{s}_{1}$, is a step-chirp in frequency from the lowest frequency, $\mathrm{f}_{1}$, up to the highest frequency, $\mathrm{f}_{16}$ (an up-chirp signal with chirp-rate $\mathrm{CR}_{1}$ ). In this case, $\mathrm{CR}_{1}$ is approximately $600 \mathrm{~Hz} / \mu \mathrm{sec}$. The sixteenth signal, $\mathrm{s}_{16}$, is the same step-chirp transmitted in reverse sequence (a down-chirp with chirp rate

$\left.-\mathrm{CR}_{1}\right)$. As the signals are constructed, the chirp-rate will continue to increase until signal eight, with $\mathrm{CR}_{8}=8 \mathrm{CR}_{1}$, and $\mathrm{s}_{8}$ composed of eight sequentially transmitted chirps. The relationship between chirp rate and signal number can be seen in Figure 3-2 ( $a$ and $b)$. 

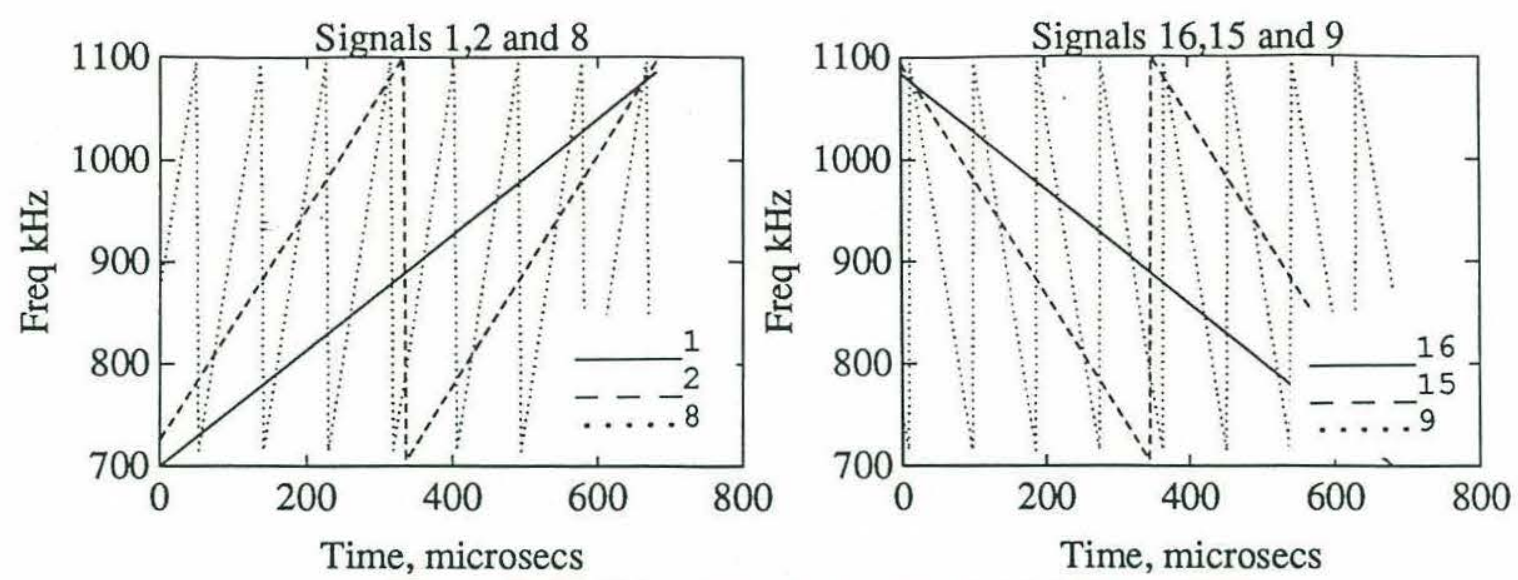

Figure 3-2: Chirp Rate for Codes 1,2,8,9,15, and 16

The set of coded signals can be described mathematically in terms of the demodulated envelope [20]:

$$
s_{i}(t)=\sqrt{2 E_{t}} \Re \varepsilon\left\{\widetilde{s}_{i}(t) e^{j 2 \pi f_{c} t}\right\} \quad i=1,2, \ldots, M
$$

where $\tilde{s}_{i}(t)$ is the complex lowpass envelope of the signal $s_{i}(t), E_{t}$ is the transmitted energy, and $f_{C}$ is the carrier frequency. The complex envelope primarily determines the imaging properties of the signals, and this leads directly to a number of important quantities. First, the signal energy (different from the transmitted energy), $\mathrm{E}$, is:

$$
E_{i}=\int_{0}^{M T} \tilde{S}_{i}(t) S_{i}^{*}(t) d t \quad i=1,2, \ldots, M
$$

It is obvious that for this class of signals, that $\mathrm{E}_{\mathrm{i}}=\mathrm{E}$ is the same for all signals in the set. For simplification, this quantity will be normalized to unity. The complex crosscorrelation coefficient between any two signals in the set is: 


$$
\rho_{i j}=\int_{0}^{M T} \widetilde{s}_{i}(t) \widetilde{S}_{j}^{*}(t) d t \quad i, j=1,2, \ldots, M ; i \neq j
$$

the cross-correlation is typically on the order of .05 for the signals in this set.

Active sonar systems operate on the principle that the transmitted signal reflects from a target and returns to a receiver. It is thus necessary to accurately model the received waveform. Given the model of the transmitted signal in (3.2.1), the received signal can be modelled in a similar manner:

$$
r_{i}(t)=\beta s_{i}\left(t-\frac{2 R(t)}{c}\right)+w(t)
$$

The received signal is a corrupted and time-delayed version of the transmitted signal, $\mathrm{Si}(\mathrm{t}) . \mathrm{R}(\mathrm{t})$ is the range of the target and $\mathrm{c}$ is the propagation speed of the medium. The attenuation factor, $\beta$, accounts for target reflectivity, attenuation by the medium, geometrical spreading, and array gain. The noise, w(t) can be modelled as an independent, zero-mean white Gaussian noise signal of spectral level $\sigma_{\mathrm{n}}^{2}$.

The target range, $\mathrm{R}(\mathrm{t})$ can be further expanded:

$$
\mathrm{R}(\mathrm{t})=\mathrm{R}+\dot{\mathrm{R}} \mathrm{t} \quad \text { where } \dot{\mathrm{R}}=\frac{\mathrm{dR}}{\mathrm{dt}}=\text { range rate of the target. }
$$

and upon substitution of the full expression for the transmitted signal, $\mathrm{s}_{\mathrm{i}}(\mathrm{t})$ from (3.2.1), we have for the returned signal:

$$
r_{i}(t)=\beta \sqrt{2 E_{t}} \Re \varepsilon\left\{\widetilde{s}_{i}\left(t-\frac{2(R+R t)}{c}\right) e^{j 2 \pi f_{c}\left(t-\frac{2(R+R t)}{c}\right)}+\widetilde{w}(t)\right\}
$$




$$
=\sqrt{2 E_{t}} \Re \varepsilon\left\{\beta e^{j 4 \pi f_{c}\left(\frac{R}{c}\right)} \widetilde{S}_{i}\left(t\left(1-\frac{2 R}{c}\right)-\frac{2 R}{c}\right) e^{j 2 \pi f_{c}\left(1-\frac{2 R}{c}\right) t}+\widetilde{w}(t)\right\}
$$

Some simplifications of this expression are possible. If it is assumed that the target has a slow range rate, then:

$$
\frac{2 \dot{R}}{c} \ll 1 \text { and therefore }\left(1-\frac{2 \dot{R}}{c}\right) \cong 1
$$

and (3.2.5) reduces to:

$$
\begin{aligned}
& r_{i}(t)=\sqrt{2 E_{t}} \Re \varepsilon\left\{\widetilde{\beta} \widetilde{s}_{i}(t-\tau) e^{j 2 \pi\left(f_{c}+f_{d}\right) t}+\widetilde{w}(t)\right\} \\
& \text { where } \tau=\left(\frac{2 R}{c}\right), f_{d}=\left(\frac{2 \dot{R}}{c}\right)_{c} \text {, and } \widetilde{\beta}=\beta e^{-j 4 \pi f_{c}\left(\frac{R}{c}\right)}
\end{aligned}
$$

Looking at the real and imaginary (in-phase and quadrature) components separately:

$$
r_{i}(t)=\sqrt{2 E_{t}} \Re \varepsilon\left\{b_{c} \widetilde{S}_{i}(t-\tau) \cos \left[2 \pi\left(f_{c}+f_{d}\right) t\right]+j b_{s} \Im_{i}(t-\tau) \sin \left[2 \pi\left(f_{c}+f_{d}\right) t\right]+\widetilde{w}(t)\right\}
$$

where $b_{c}$ and $b_{s}$ are the real and imaginary parts of the complex coefficient $\beta$, and are modelled as independent, zero-mean Gaussian random variables with variance $\sigma_{\mathbb{B}}{ }^{2}$. This model has been widely used for the underwater channel $[11,12]$. The complex coefficient $\beta$ is characterized by a uniform phase distribution between $-\pi$ and $\pi$, and a Rayleigh envelope, where:

$$
E\{|\beta|\}=\sqrt{\frac{\pi}{2}} \sigma_{\beta}^{2} \text { and } E\left\{|\beta|^{2}\right\}=2 \sigma_{\beta}^{2} \text {. }
$$

This relationship leads directly to the expected value of the received energy, $\mathrm{E}_{\mathrm{r}}$ :

$$
E\left\{E_{r}\right\}=E\left\{\widetilde{\beta E}_{t}\right\}=2 \sigma_{\beta}^{2} E_{t}
$$




\subsection{Signal Performance in an Imaging Scenario}

The design criteria stated in (1.1) and (1.2), and the resulting coded signals are sufficient only to describe the performance of the signals in a static imaging scenario (i.e. stationary transmitter, stationary receiver, and stationary targets). The introduction of a doppler frequency shift introduces another dimension into the detection and signal separation problem. While the operator can guarantee that the system related components will not move, there is no way that a non-moving target can be guaranteed.

Because this is the case, the signals not only must be separable under all time shifts, but also must be separable under all doppler frequency shifts that may arise due to relative motion between the target and the sonar.

In a simple receiver, the returned signal is passed through a matched filter with an impulse response matched to the transmitted signal. The time-delay corresponding to the maximum response of the filter leads to an estimate of the target's range. To accommodate the possibility of target velocity, the single filter is expanded into a bank of matched filters. Each filter in the bank is tuned to a doppler-shifted replica of the transmitted signal. This more complex receiver will pass the returned signal through all the filters in the bank; the filter which gives the largest response leads to the estimate for target motion (i.e. if the zero-doppler shifted filter gave the largest response, the target would be estimated to have zero range-rate). In the case of multiple waveform transmission, where it is desired to separate the returned waveforms under all circumstances, it is important that a doppler- shifted version of one signal not be confused with another signal.

If it is assumed that the returned waveform is uncorrupted by noise, and that there is no signal attenuation, then the simplified model of the returned signal is, from (3.2.6): 


$$
\mathrm{r}_{\mathrm{i}}(\mathrm{t})_{\text {simp }}=\sqrt{2 \mathrm{E}_{\mathrm{t}}} \Re \varepsilon\left\{\mathrm{s}_{\mathrm{i}}(\mathrm{t}-\tau) \mathrm{e}^{\mathrm{j} 2 \pi\left(\mathrm{f}_{\mathrm{c}}+\mathrm{f}_{\mathrm{d}}\right) \mathrm{t}}\right\}
$$

Examining this expression, there are two nonrandom parameters that are related to the target: the delay time, $\tau_{\mathrm{t}}$, and the doppler frequency shift, $\mathrm{f}_{\mathrm{dt}}$. Sending this simplified model of the returned signal through the filter bank, we get the response:

$$
L_{i, j}\left(\tau_{t}, \tau: f_{d t}, f_{d}\right)=\int \sqrt{2 E_{t}} \Im_{i}(t-\tau) e^{j 2 \pi f_{d t} t_{\Upsilon_{j}}^{t}(t-\tau)} e^{-j 2 \pi f_{d} t} d t
$$

where $\tau_{t}$ and $f_{d t}$ are the true time-delay and doppler shift for the returned signal $i$, and $\tau$ and $f_{d}$ are the time-delay and doppler shift associated with the impulse response of the filter matched to signal $j$. Examination of the squared envelope of the response, $L_{i, j}{ }^{2}$, leads to the range-doppler ambiguity function, $\Theta\left(\tau_{\mathrm{t}}, \tau: \mathrm{f}_{\mathrm{dt}}, \mathrm{f}_{\mathrm{d}}\right)$ (since the complex envelope of the signals will be assumed throughout the thesis, the tilde superscript $(\sim)$ will henceforth be dropped):

$$
\begin{aligned}
& L_{i, j}^{2}\left(\tau_{t}, \tau: f_{d t} f_{d}\right)=E_{t} \widetilde{\beta}^{2} \Phi_{i, j}^{2}\left(\tau_{t}, \tau: f_{d t}, f_{d}\right) \mid \\
& \text { where } \Phi_{i, j}\left(\tau_{t}, \tau: f_{d t}, f_{d}\right)=\int_{i}\left(t-\tau_{t}\right) s_{j}^{*}(t-\tau) e^{j 2 \pi\left(f_{d t}-f_{d}\right) t} d t \\
& \text { and } \Theta_{i, j}\left(\tau_{t}, \tau: f_{d t}, f_{d}\right)=\left|\Phi_{i, j}^{2}\right|
\end{aligned}
$$

The detailed properties of this function are given in Van Trees [12] and Rihaczek [13]. Some of the basic principles will be outlined here to explore the performance of the coded signals. First, a slight adaptation is necessary for the code-division case, where many signals are being transmitted and processed simultaneously. In (3.3.4), $\Theta_{i, j}$ for $i=j$ will be referred to as the auto-ambiguity function, and $\Theta_{i, j}$ for $i \neq j$ will be re- 
ferred to as the cross-ambiguity function. Having made this slight expansion of the traditional analysis, it is possible to determine the performance of the code-division sonar.

Combining (3.2.6) and (3.3.3), the expected value of the function $\mathrm{L}_{\mathrm{i},{ }^{2}}{ }^{2}$ can be found:

$$
E\left\{L_{i, j}^{2}\left(\tau_{t}, \tau: f_{d t}, f_{d}\right)\right\}=2 \sigma_{\beta}^{2} E_{t} \Theta_{i, j}\left(\tau_{t}, \tau: f_{d t}, f_{d}\right)
$$

which indicates that the received energy is directly dependent on the properties of the range-doppler ambiguity function. The ambiguity function is closely linked to the radar uncertainty principle in that the volume under the surface of the function is conserved. Since the signal energy was normalized to unity in (3.2.2), it follows that:

$$
\int \Theta_{i, i}\left(\tau_{t}, \tau: f_{d t}, f_{d}\right) d \tau d f_{d}=1
$$

The desired characteristics of the auto-ambiguity function of a signal are a single sharp peak at $\left(f_{d t}-f_{d}\right)=0$ and $\left(\tau_{t}-\tau\right)=0$, and minimal value for all other points. This allows a more precise estimation of the actual time delay and doppler shift of the reflected signal. Any peaks in the function that are displaced from this point could lead to an incorrect estimation of the range and motion of the target. Since the multi-beam sonar system uses the principle of code-division, it is also desired to guarantee that a doppler- and time-shifted version of one signal will not be detected as a version of a different signal. Mathematically, this requires that all cross-ambiguity functions for the set are "smeared" over the $\tau-f_{d}$ plane, so that no peaks can be distinguished. The coded signals generated for this system posses these properties. As examples, figure 3-3 shows the auto-ambiguity functions for signals eight and twelve. 


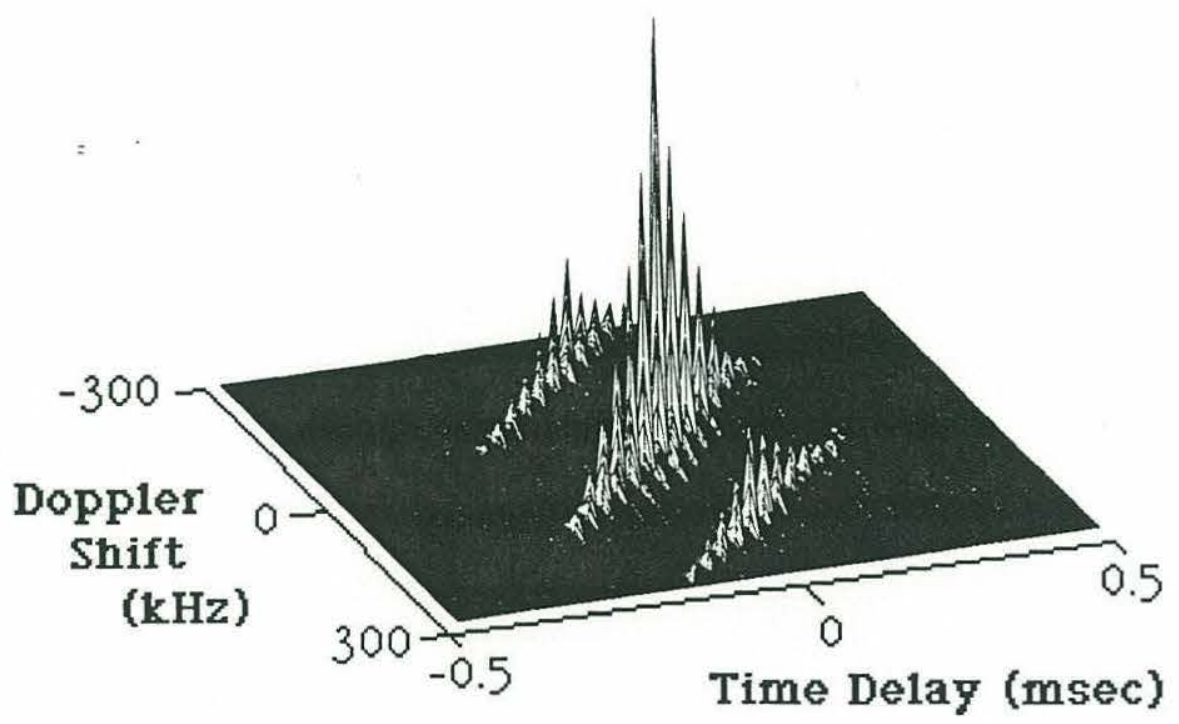

(a)

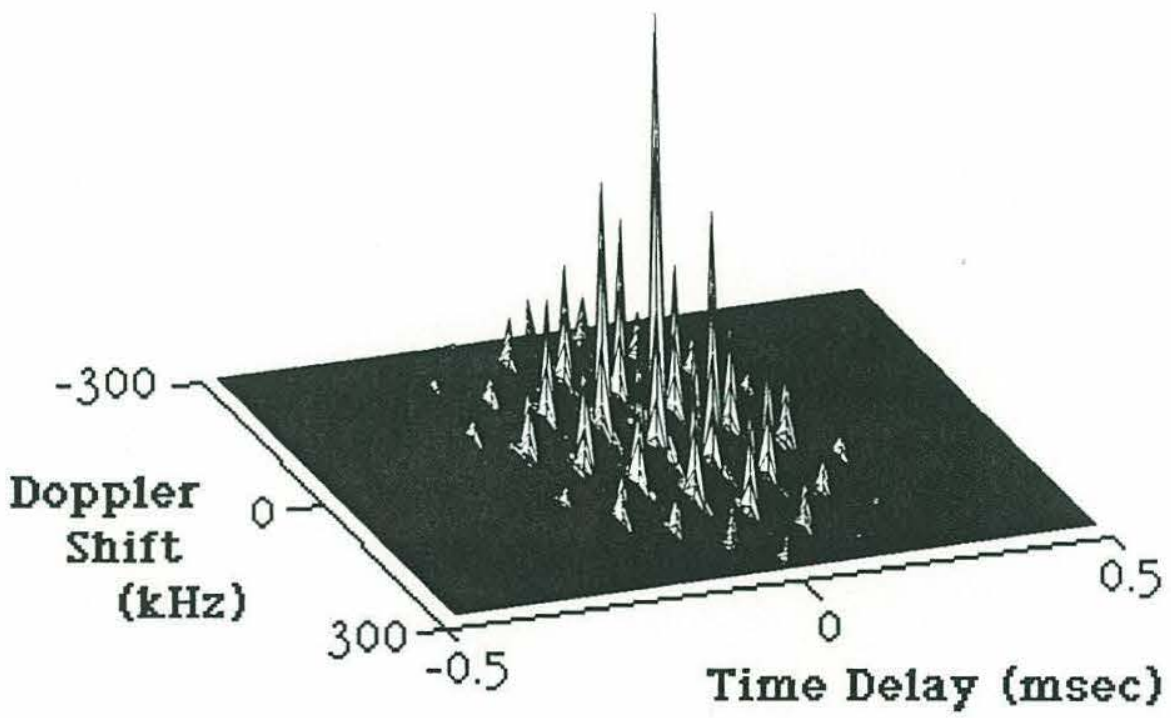

(b)

Figure 3-3: Auto-Ambiguity Functions for Signals Eight (a), and Twelve (b)

The general performance of $s_{8}$ and $s_{12}$ is representative of all signals in the set. They are all maximized for $\left(\tau_{\mathrm{t}}-\tau\right)=0$ and $\left(\mathrm{f}_{\mathrm{dt}}-\mathrm{f}_{\mathrm{d}}\right)=0$, and minimized over much of the remaining plane. There is a global ambiguity in all the auto-ambiguity functions. This 
arises from the step-chirp nature of the signals. Any of the signals that is dopplershifted by exactly $25 \mathrm{kHz}$, will match some of the unshifted portions of the original signal. This is most apparent for $\mathrm{s}_{1}$ and $\mathrm{s}_{16}$, where the number of chips that match between an original signal and a doppler-shifted by $25 \mathrm{kHz}$ is fifteen (this yields 0.94 for a peak/sidelobe ratio, the worst in the set). The global ambiguity in the delay-doppler plane is related to the chirp-rate of the signal. Figure 3-4 shows that the basic structure of the signals is a set of sharp peaks whose width is proportional to the length of a chip. These peaks are contained inside a wider envelope, whose width is proportional to the length of the entire signal. This envelope is 'skewed' in the plane with a slope that is related to the chirp rate. As the signals increase in chirp rate from $\mathrm{s}_{1}$ to $\mathrm{s}_{8}$, the slope of the envelope increases (signals $s 9-s_{16}$ have the same properties with a negative slope for the envelope). 

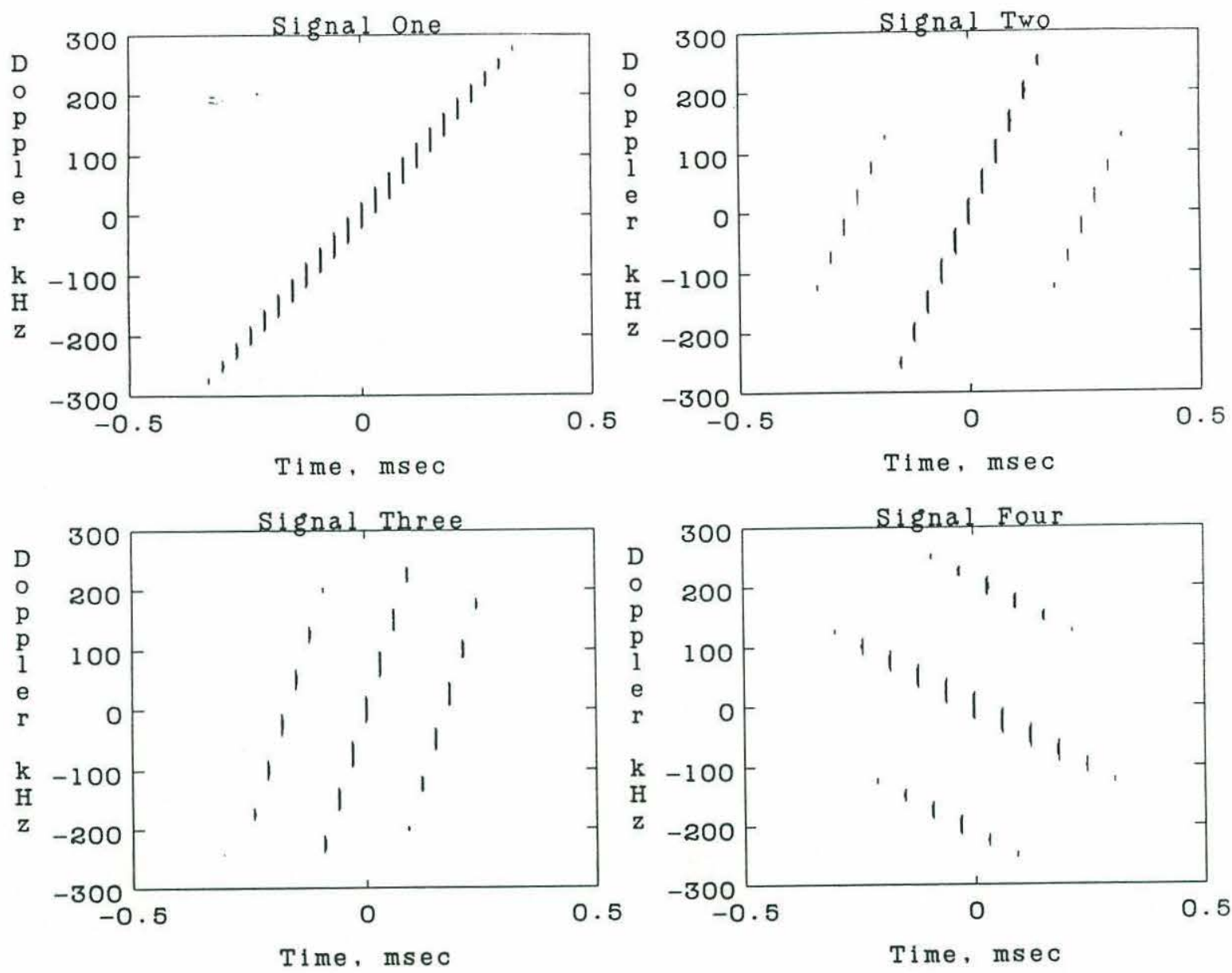

Figure 3-4: Contours of the Auto Ambiguity Functions for Signals 1, 2, 3, and 4

The cross-ambiguity functions of the signals exhibit the desired property of being well-distributed over the delay-doppler plane, with no distinct peaks. The maximum value of the cross-ambiguity functions is typically $17 \mathrm{~dB}$ lower than the peak of the auto-ambiguity functions. Figures 3-5 and 3-6 show examples of typical cross-ambiguity functions for this signal set. The structure of the cross-ambiguity functions ensures that there is little probability of confusion between doppler shifted versions of different signals. The code-division property of these signals holds under both time- and doppler-shifts. 


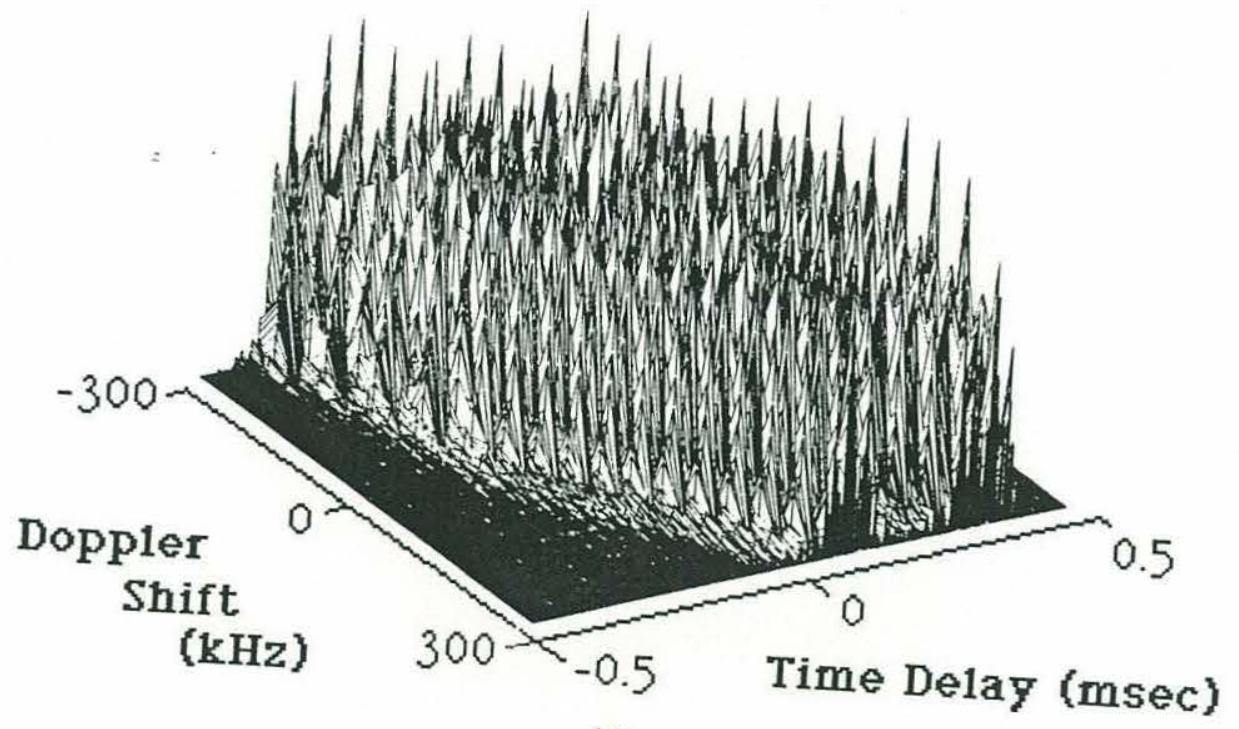

(a)

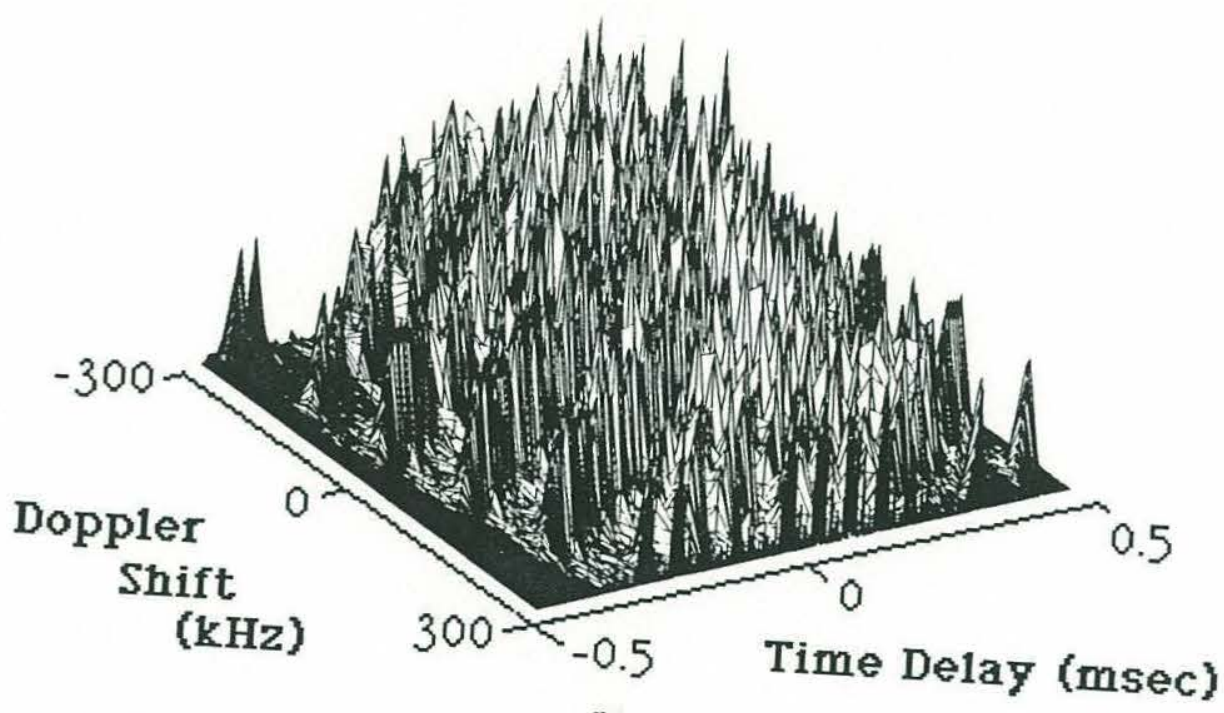

Figure 3-5: Cross Ambiguity Functions for Signals One/Two (a), and Four/Eight (b) 


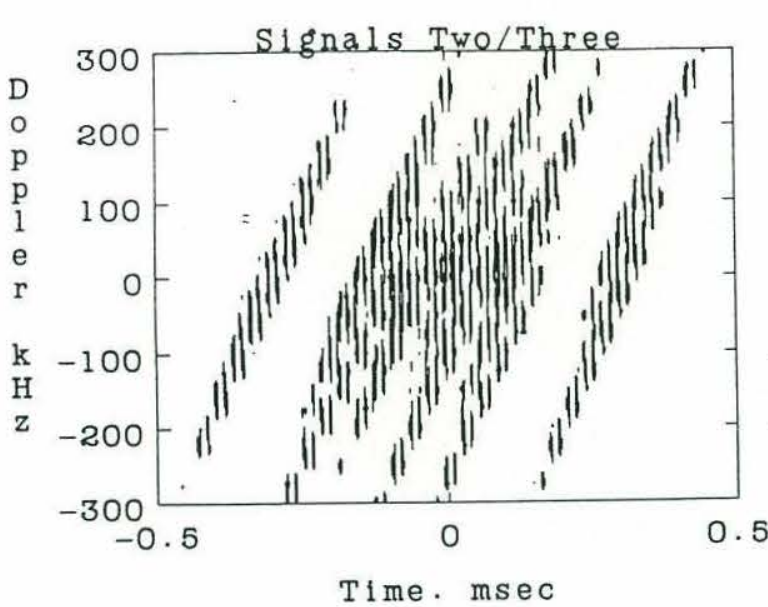

(a)

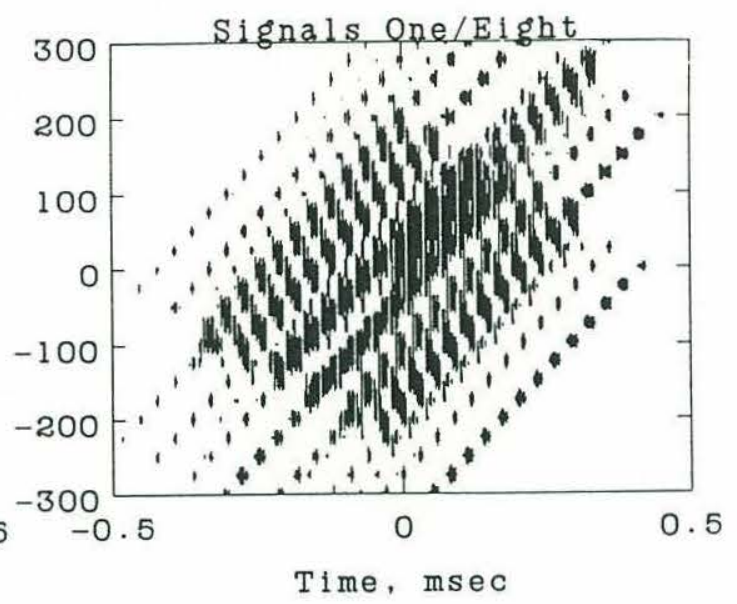

(b)

Figure 3-6: Contours of the Cross-Ambiguity Functions for Signals Two/Three (a) and Signals One/Eight (b).

The resolution limits for a target's range and motion are determined from the auto-ambiguity function. Along the time-delay axis, the shape of the auto-ambiguity function is a squared triangle, resulting from the correlation of two rectangular pulses of length $\mathrm{T}$. The width of this correlation peak is $2 \mathrm{~T}$, or $2(40 \mu \mathrm{secs})=80 \mu \mathrm{secs}$. Along the doppler axis, the function is a $\sin (\mathrm{x}) / \mathrm{x}$ function with the first null appearing at $25 \mathrm{kHz}$. Therefore, the sequential-rectangular signals can resolve a target's range to $12 \mathrm{~cm}$ and its range-rate to $21 \mathrm{~m} / \mathrm{sec}$.

Examination of the auto- and cross- ambiguity functions for the set of signals used in this sonar system has shown that the code-division principle will work when applied to a realistic imaging task. The global ambiguity associated with receiving a doppler-shifted signal, coupled with poor resolution of target doppler indicates that modifications would be necessary to accommodate situations where high range rates are expected. In order to improve performance for this task, a wider frequency separation between the chips is required. With this restriction, there are still a wide variety of applications in which the signals can be effectively used for code-division imaging. 


\section{Chapter 4}

\section{Receiver Design}

\subsection{Signal Conditioning}

During the sequence of transmission,propagation, reflection from a target, and reception, an acoustic wave is subject to a number of processes that degrade all or parts of the signal. These processes include the addition of noise, non-uniform amplification by the sonar electronics, attenuation and spreading in the medium, and non-uniform hydrophone characteristics. These factors are highly dependent on the frequency of the acoustic wave. One feature of frequency-hop coding is the use of a wide frequency bandwidth. Within the signals, different chips will be subjected to different attenuation mechanisms. Many of these processes can be accurately anticipated and compensated for by pre-processing the signal. This pre-conditioning phase is the first stage of the receiver.

Some of the mechanisms that degrade the signal, such as signal attenuation and noise addition are well known, and are routinely compensated for by applying a timevarying gain (TVG) and a bandpass filter (BPF) upon reception. The pre-conditioning stages of the receiver is schematically shown in Figure 4-1. 


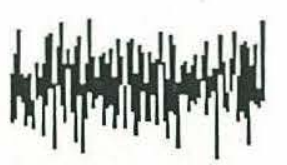

Received Signal

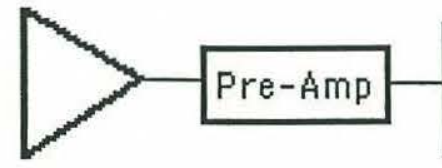

Hydrophone

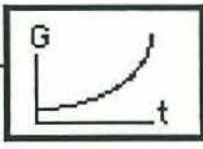

TVG

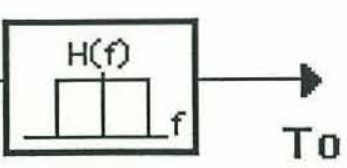

BPF Receiver

Figure 4-1: Steps Taken to Pre-condition the Received Signal

One of the primary mechanisms for signal attenuation is absorption of energy by the water. This effect has been thoroughly documented [18] and is strongly dependent on the frequency of propagation. Energy is absorbed by the medium according to the relation:

$$
E(r)=E_{t} e^{-\alpha\left(f, T_{w}\right) r}
$$

where $\mathrm{E}(\mathrm{r})$ is the energy at range $\mathrm{r}$ from the transmitter, $\mathrm{E}_{\mathrm{t}}$ is the originally transmitted energy, as before, and $\alpha\left(f, T_{w}\right)$ is the reciprocal absorption length, in $\mathrm{m}^{-1}$. As indicated, the magnitude of $\alpha\left(f, T_{w}\right)$ is dependent on both frequency, $f$, and water temperature, $T_{w}$. For the situation considered here, the primary concern will be to examine the variation due to frequency. The variation with temperature will be only peripherally addressed.

Over the range from $0 \sim 500 \mathrm{kHz}$, the absorption mechanism in the ocean is dominated by the molecular relaxation time constants of Boron $(0 \sim 5 \mathrm{kHz})$ and Magnesium Sulfate $(5 \mathrm{kHz} \sim 500 \mathrm{kHz})$. Above $500 \mathrm{kHz}$, the dominant factor is the structural relaxation of the water molecules, as in fresh water. In this region, experimental evidence [14] has shown that the behavior of $\alpha$ can be described as follows:

$$
\alpha\left(f, T_{w}\right) \cong(3.2)\left(\frac{4 v(2 \pi f)^{2}}{3 c^{3}}\right)
$$




$$
\cong 6.77 \times 10^{-6}\left(\frac{f^{2}}{f_{r}\left(T_{w}\right)}\right) \text {. }
$$

where $v$ is the kinematic viscosity of water, $\mathrm{f}$ is the propagation frequency, $\mathrm{c}$ is the propagation speed of the medium, $\mathrm{f}_{\mathrm{r}}\left(\mathrm{T}_{\mathrm{W}}\right)$ is the characteristic relaxation frequency of the medium at a given temperature. Figure 4-2 shows a plot of $\alpha$ vs frequency for a water temperature of $5^{\circ} \mathrm{C}$.

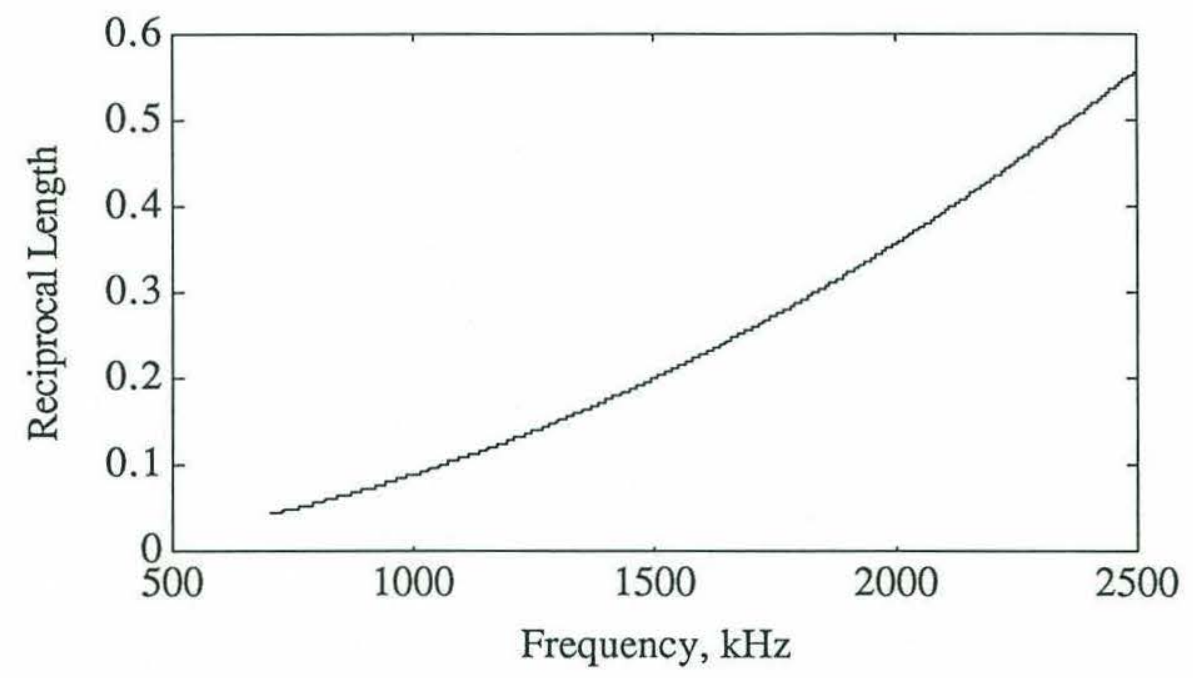

Figure 4-2: Reciprocal Absorption Length $\left(\mathrm{m}^{-1}\right)$ vs. Frẹquency $(\mathrm{kHz})$.

The maximum operating range of an imaging sonar can be approximated by finding that range where the signal energy is reduced by $10 \mathrm{~dB}$ due to absorption alone. Substitution of this relation into (4.1.1) yields an maximum range of about 25 meters at $900 \mathrm{kHz}$. Operation beyond this range will require a lower carrier frequency in order to reliably image a target.

Another attenuation mechanism is the geometric spreading of the acoustic wave from the transmission source. At the frequencies and ranges applicable to code-division imaging, the wavefront spreads spherically away from the source, resulting in a $1 / \mathrm{r}^{2}$ attenuation loss. The combination of geometrical spreading and absorption can be summed up in the total attenuation equation (plotted in Fig 4-3): 


$$
E(r)=\frac{E_{t}}{r^{2}} e^{-\alpha\left(f, T_{w}\right) r}
$$

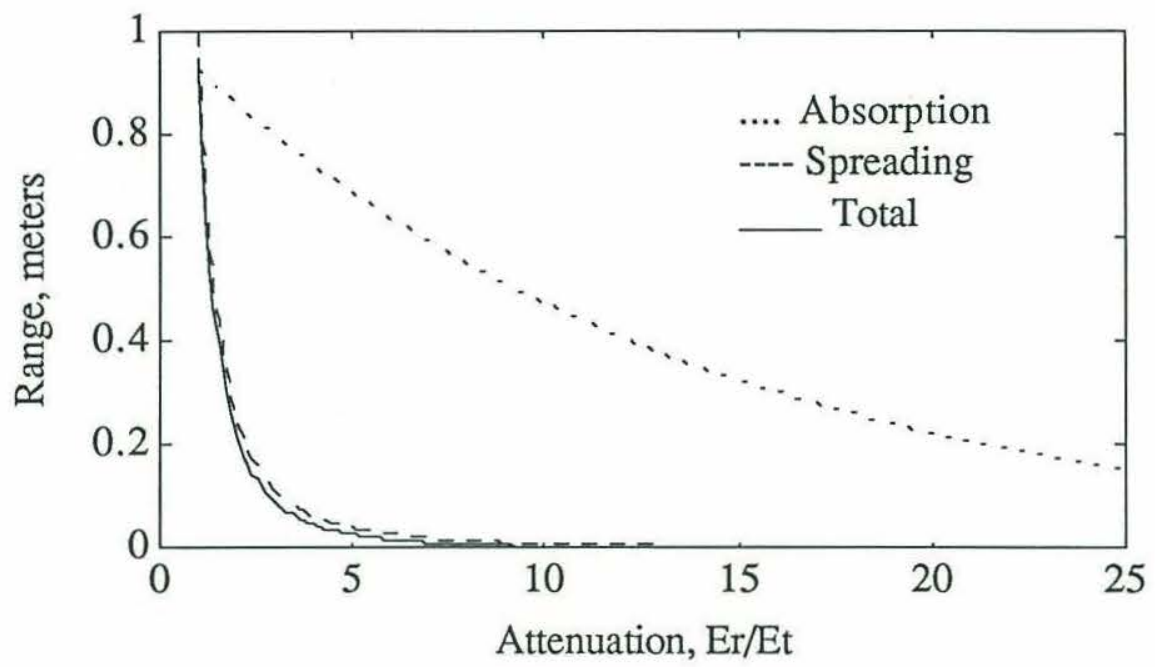

Figure 4-3: Attenuation of Signal Energy by the Medium (at $900 \mathrm{kHz}, 5^{\circ} \mathrm{C}$ )

As can be seen from the figure, the dominant effect is the spherical spreading of the wavefront.

Complete compensation of these effects is a two-dimensional process in range and frequency. In order to separate these effects as much as possible, an average reciprocal absorption length, $\alpha$, will be used to quantify the average effects of absorption across the signal bandwidth. This will be combined with the geometrical spreading effect to give a frequency-independent attenuation factor dependent on range only:

$$
b(r)=\frac{e^{-\bar{\alpha} r}}{r^{2}}
$$

Where $\alpha$ is the average reciprocal absorption length over the bandwidth of $700 \mathrm{kHz}$ to $1100 \mathrm{kHz}$. The frequency dependent compensation is expressed by: 


$$
b(f, r)=e^{-\alpha^{\prime}(f) r}
$$

In this expression, $\alpha^{\prime}$ is the deviation from the mean, $\left(\alpha^{\prime}=\alpha-\alpha\right)$. It is worthwhile to consider the contribution of these two effects separately. At the maximum operating range of $25 \mathrm{~m}$, with $\alpha=.074 \mathrm{~m}-1, \mathrm{~T}_{\mathrm{w}}=5^{\circ} \mathrm{C}$, the attenuation $\mathrm{E}_{\mathrm{r}} / \mathrm{E}_{\mathrm{t}}$ from (4.1.4) is 2.512 $x 10^{-4}$, or $-36 \mathrm{~dB}$. The actual attenuation at each extreme of the bandwidth is $5.33 \times 10^{-4}(-33 \mathrm{~dB})$ at $700 \mathrm{kHz}$ and $1.07 \times 10^{-4}(-40 \mathrm{~dB})$ at $1100 \mathrm{kHz}$. The approximate expression in (4.1.4) is accurate to within a factor of two parts in ten thousand. This is a consequence of the fact that the geometrical spreading, which is frequency-independent, dominates the attenuation (as previously shown in Figure 4-3). Because of this accuracy, the attenuation by the medium will be solely approximated by (4.1.4), and frequency dependent effects of the medium will be ignored. The TimeVarying Gain that will be applied to the signal is, from (4.1.4):

$$
\operatorname{TVG}(\mathrm{t})=\frac{1}{\mathrm{~b}(\mathrm{r})}=\frac{1}{\mathrm{~b}(\mathrm{ct})}=(\mathrm{ct})^{2} \mathrm{e}^{(\bar{\alpha} \mathrm{c}) \mathrm{t}}
$$

where $\mathrm{c}$ is the propagation speed, as before.

There are other effects which serve to degrade the received signal. The presence of noise in the measurements due to noise in the environment and in the sonar electronics can be partially compensated. More frequency-dependent effects arise from the non-uniform gain of amplifiers, hydrophones, and transducers in the sonar. The effects of these mechanisms can be reduced by an appropriately constructed filter that will eliminate noise not in the bandwidth of interest, as well as correct the non-uniform performance of the sonar within the signal bandwidth.

Obtaining a mathematical representation that describes the transfer function for the ensemble of all the components in the sonar can be a difficult. It is more direct and more accurate to obtain an estimate of the response of the sonar directly from repeated 
measurements. This was done for the multiple-beam sonar by transmitting and receiving many signals and examining the response over all returns. A response function was estimated by averaging over 120 transmit/receive cycles. From this estimate the non-uniform response of the sonar was inverted and used to design a filter to pre-condition the received signals.

The sonar system has a peak response at $750 \mathrm{kHz}$, which falls off by $20 \mathrm{~dB}$ at $1100 \mathrm{kHz}$. Using the data gathered from the 120 trial signals, a Finite Impulse Response bandpass filter was designed using the window technique [15]. Figure 4-4 (a) shows the power spectral density function of a typical received signal. The signal was reflected off a weak target, resulting in $6 \mathrm{~dB}$ Signal to Noise Ratio. The effectiveness of the filter is shown in Figure 4-4 (b). The noise in the stopband has been attenuated by approximately $44 \mathrm{~dB}$, and the variance inside the passband has been reduced by the variable gain of the filter inside the passband. The conditioned signal exhibits only a $5 \mathrm{~dB}$ difference between the peak response and the minimum response inside the passband.
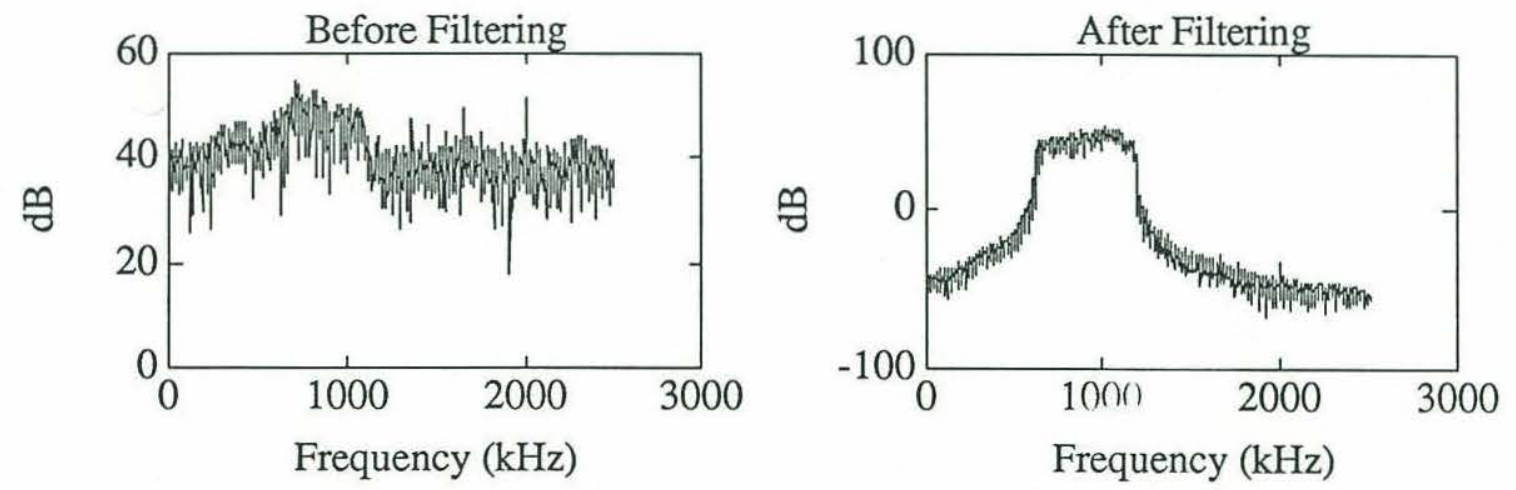

Figure 4-4: Power Spectral Density Function of Received Signals Before (a), and After (b) Filtering 


\subsection{Signal Detection}

\subsubsection{Design of the Signal Detector}

Although signal-to-signal rejection is essential to the performance of the multibeam system, each signal must also be independently capable of accurately conveying information about the target from which it is reflected. The performance of the signals is dependent on many things such as signal design, receiver design, noise level, and target reflectivity. Two factors over which the operator has direct control are the signal and receiver design. Having discussed the design of the signals and the pre-conditioning stage, the optimum receiver for these signals can now be found.

Returning to the model of the received signal in (3.2.7), there are a number of modifications to the originally transmitted signal. Correctly determining these factors is crucial to the performance of the system. Accurately estimating the time delay and doppler shift in the presence of noise is directly related to determining the target's location and range rate. The receiver will be designed for the zero doppler signal.. The doppler performance of the signals was discussed in Chapter Three. Once designed, the zero-doppler receiver can be easily tuned to accommodate a wide range of possible doppler shifts. The accuracy to which the phase distortion can be determined is central to the performance of the receiver. There are several models that examine the dependence of phase on receiver performance. Two models will be employed here:

1) The coherent model, which will assume that full knowledge of the phase distortion is available and 2) The incoherent model for which no knowledge of the phase is assumed.

If the exact phase distortion is known (coherent model), the detection strategy for coherent reception is simple. A decision variable is constructed from the received 
signal, and a decision is made based on the magnitude of the decision variable. Stated in probabilistic terms, the goal is to determine

$$
\operatorname{Pr}\{\text { signal } i \text { was transmitted } \mathrm{I}(\mathrm{t}), \mathrm{t} \leq \mathrm{t} \leq \mathrm{t}+\mathrm{MT}\},
$$

and choose the signal that was most probable given the received signal. This is the well known maximum a-posteriori decision rule, and the decision variable, $\zeta_{\mathrm{i}}$, has the form:

$$
\zeta_{i}=R \varepsilon\left\{\int_{t}^{t+M T} e^{j \phi(t)} r(t) s_{i}^{*}(t) d t\right\} \quad i=1,2, \ldots, M
$$

The decision rule requires that the completely known phase distortion $e^{-j \phi(t)}$ be removed by applying an exactly inverse phase shift. The phase-corrected signal is then correlated with all sixteen transmitted waveforms. The best estimate of the signal that is was reflected at time $t$ is the signal that yields the largest decision variable. The probability for a correct decision in this case is:

$$
\mathrm{P}_{\mathrm{c}}=\operatorname{Pr}\left\{\zeta_{\mathrm{i}}>\zeta_{\mathrm{k}} \mid \zeta=\zeta_{\mathrm{i}}\right\} \quad \mathrm{k}=1,2, \ldots, \mathrm{M} ; \mathrm{k} \neq \mathrm{i}
$$

which is (assuming that all signals are equally probable a-priori):

$$
\begin{aligned}
& P_{c}=\frac{1}{2 \pi} \int_{-\infty}^{\infty} d \zeta \exp \left\{\frac{-(\zeta-\sqrt{2 \sigma})^{2}}{2}\right\}\left[\int_{-\infty}^{\zeta} d y \exp \left\{\frac{-y^{2}}{2}\right\}\right]^{M-1} \\
& \text { where } \sigma=\frac{E_{r}}{\sigma_{n}^{2}}=\frac{\widetilde{\beta}^{2} E_{t}}{\sigma_{n}^{2}}
\end{aligned}
$$

is the signal to noise ratio at the receiver. The probability of a detection error is $\mathrm{Pe}_{\mathrm{e}}=$ 
1- $\mathrm{P}_{\mathrm{C}}$. Figure 4-5 shows the coherent correlation of coded signal eight $\left(\mathrm{s}_{8}\right)$ with all sixteen waveforms in the set. The degree of signal-to-signal rejection for the codes is apparent.

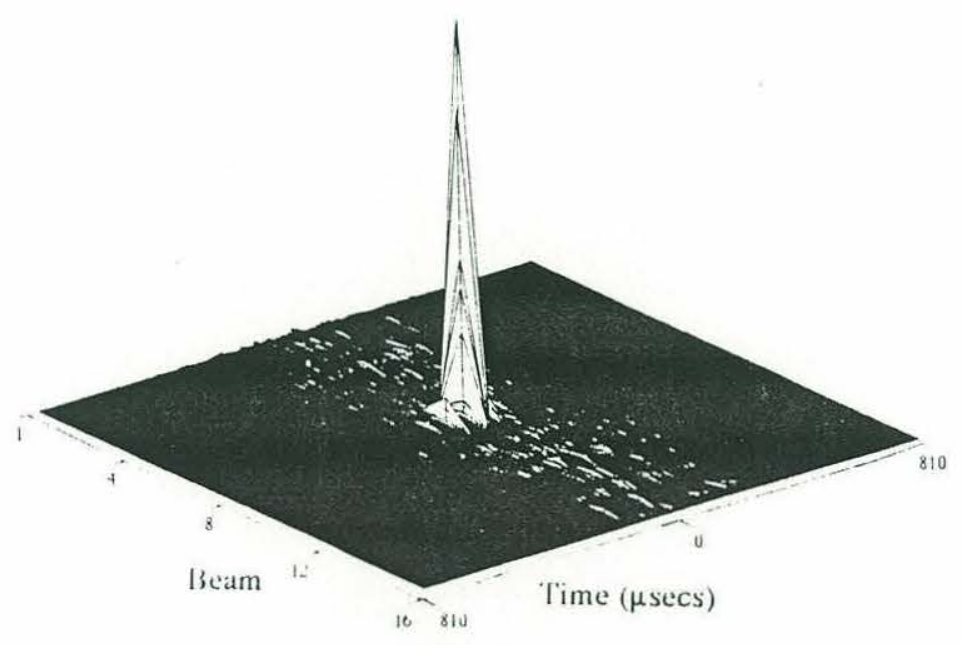

Figure 4-5: Coherent Detection for Signal Eight

The coherent receiver, although designed on the assumption that the phase distortion is completely known, is quite robust to small unknown deviations in the phase of the signal. This is particularly true if the received signal is a phase-shifted version of the transmitted signal. In this case, all the internal phase structure of the signal is completeiy preserved, and the decision variable found in (4.2.1) is still optimum. In fact, the phase shift ej $\phi(t)$ can be completely disregarded in this case, and a modified decision variable can be constructed:

$$
\zeta_{i}^{\prime}=\Re \varepsilon\left\{\int_{t}^{t+M T} r(t) s_{i}^{*}(t) d t\right\} \quad i=1,2, \ldots, M
$$

This variable will asymptotically yield the same performance as (4.2.1) as the transmitted signals are composed of an increasing number of cycles. The difference will be a slight shift in the correlation peak where

$$
\mathrm{t}_{\text {shift }} \leq \frac{\lambda}{\mathrm{c}}
$$


For simplicity's sake, given the fact that the transmitted codes are composed of more than 1000 cycles, the coherent decision variable will be stated as in (4.2.4).

For the case of a time-varying phase distortion, the coherent receiver is still robust as long as the magnitude of the change is small. This can be appreciated more intuitively in the decision-space for signal detection. The decision rule partitions the space into $\mathrm{M}$ regions, one region for each of the signals. The decision variable is a vector in that space. If the decision vector falls into region $m$, then signal $\mathrm{s}_{\mathrm{m}}(\mathrm{t})$ is detected. If the phase of the signal is changed by a small angle $\mu$ the vector is rotated by that angle. The performance of the decision rule is affected on the order of $(1-\cos (\mu))$, which is small for small angles $\mu$.

In most imaging environments, one cannot guarantee complete knowledge of the phase distortion, or even that the distortions will be small. Often the phase is wildly perturbed by reflection from the target and the properties of the medium. For this case, the incoherent receiver only requires that the internal phase structure of a frequency chip be preserved. The incoherent receiver for the frequency hop signals is quite different from the coherent case. The basic principle of this receiver is to construct a bank of band-pass filters, each tuned to the frequency for the different chips in the signals, $\mathrm{c}_{\mathrm{k}}$ $(\mathrm{k}=1,2, . ., \mathrm{M})$. As the signal is received, it is "de-hopped" by separating the signal into sixteen chips, $c_{j}(j=1,2, . ., M)$. Each chip is then passed separately through the bank of filters, and the square magnitude of the response from each filter is stored. The total number of squared responses is $16^{2}=256$. The responses are then decoded and summed for each possible coded permutation to form the decision variable. Mathematically, the decision variable is then:

$$
\zeta_{i}=\sum_{P_{i}(j, k)=1}^{M}\left|\int_{0}^{T} c_{r j}(t) c_{k}^{*}(t) d t\right|^{2} \quad j, k=1,2, \ldots, M
$$




$$
\text { where the summation } \sum_{P_{i}(\mathrm{j}, \mathrm{k})=1}^{M}
$$

is the decoded sum for the signal $s_{i}(t)$. The performance of the incoherent receiver cun also be evaluated. By squaring the response and the adding them, the performance of the receiver is degraded by an effect known as the incoherent combining loss. The probability density function of the decision variable is now a chi-squared density function with thirty-two degrees of freedom. The probability of making a correct decision, still generally described by (4.2.2), is (as expressed in [11]):

$$
\int_{0}^{\infty} d \zeta\left(\sigma \zeta^{\mathrm{M}-1}\right) \exp \{-\sigma(1+\zeta)\} \mathrm{I}_{\mathrm{M}-1}(-\sigma \sqrt{\zeta})\left[1-\exp \{-\sigma \zeta\} \sum_{\mathrm{j}=0}^{\mathrm{M}-1} \frac{1}{\mathrm{j} !} \zeta^{\mathrm{j}}\right]^{\mathrm{M}-1}
$$

where $\mathrm{I}_{\mathrm{M}-1}(\mathrm{x})$ is the modified Bessel function of order $(\mathrm{M}-1)$ of $\mathrm{x}$, and $\sigma$ is the signal to noise ratio at the receiver, as before. The performance curves for the coherent and incoherent receivers are shown graphically in Figure 4-6.

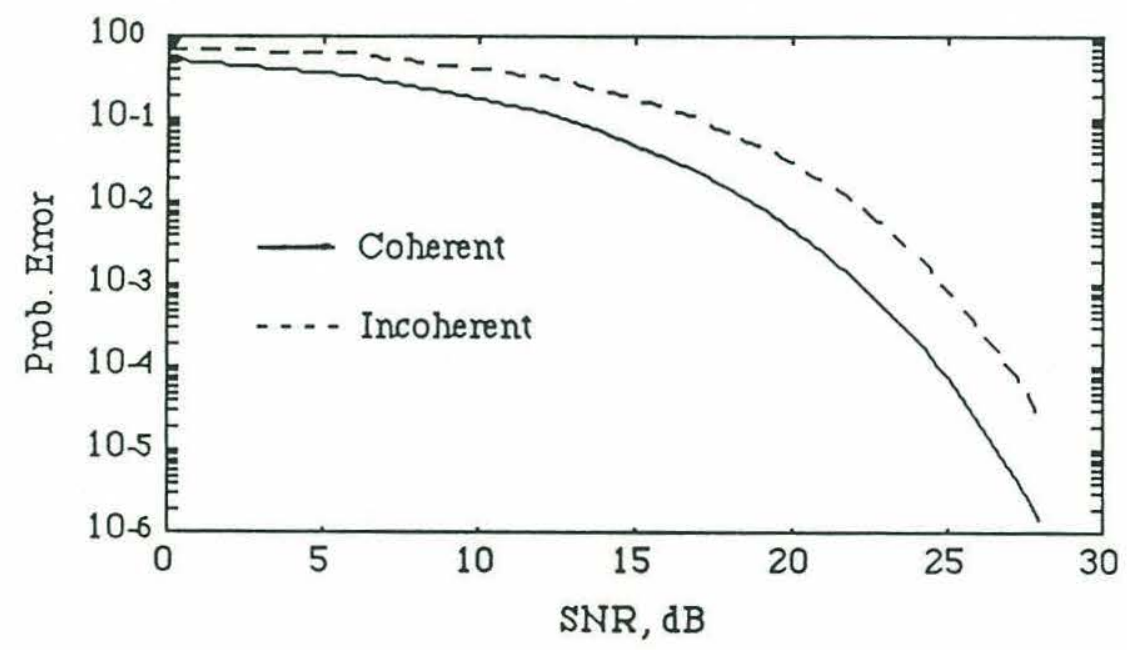

Figure 4-6: Probability of Decision Error for the Coherent and Incoherent Receivers vs. Signal to Noise Ratio.

The optimal receiver for detection of the individual signals is the first step towards the multiple parameter estimation problem that lies at the heart of locating a target 
in the ensonified aperture. It is not realistic to assume that a target inside this aperture will reflect a single signal and be transparent to all the others. Instead, the target will reflect the energy of all the signals simultaneously. The individual reflection will vary in energy depending on where the target is located in the beam patterns of the ensonified aperture. Estimating the energy of each of the reflected signals is the subject of Sections 4.3 and 4.4 .

\subsubsection{Further Developments in Signal Design for Incoherent Reception}

It is possible to improve the performance of the incoherent receiver by modifying the design of the signals. Each chip is a windowed version of an infinite sinusoid. In the signals designed so far, the window function has been rectangular with length $\mathrm{T}$, the chip length. It is well known [16] that while the rectangular window provides excellent resolution, for many purposes it is undesirable due to the spectral leakage that results. The literature is rich with analysis of different windows designed to determine the best tradeoff between resolution and stability. For this system, a Hanning window will be used. The Hanning window of length T has the form:

$$
W(t)=\sqrt{\frac{8}{3 T}}\left(\frac{1}{2}+\frac{1}{2} \cos \left(\frac{2 \pi t}{T}\right)\right)
$$

The highest sidelobe is $-32 \mathrm{~dB}$ down from the peak (compared to $-13 \mathrm{~dB}$ for the rectangular window), and the resolution in the frequency domain is lower by a factor of two (i.e. the main lobe is twice as wide). In order to recover the desired frequency resolution, it is necessary to make the chip length twice as long. This has the undesirable effect of degrading the range resolution. Also, since the window function is small at the two endpoints, the signals vary widely in power during the course of transmission. 
Thus, for a power limited system, the best choice might be the rectangular window to maximize the transmitted energy. Figure 4-7 graphically illustrates the tradeoff between range resolution and sidelobe reduction for a rectangular window of length T, a Hanning window of length $\mathrm{T}$, and a Hanning window of length $2 \mathrm{~T}$.
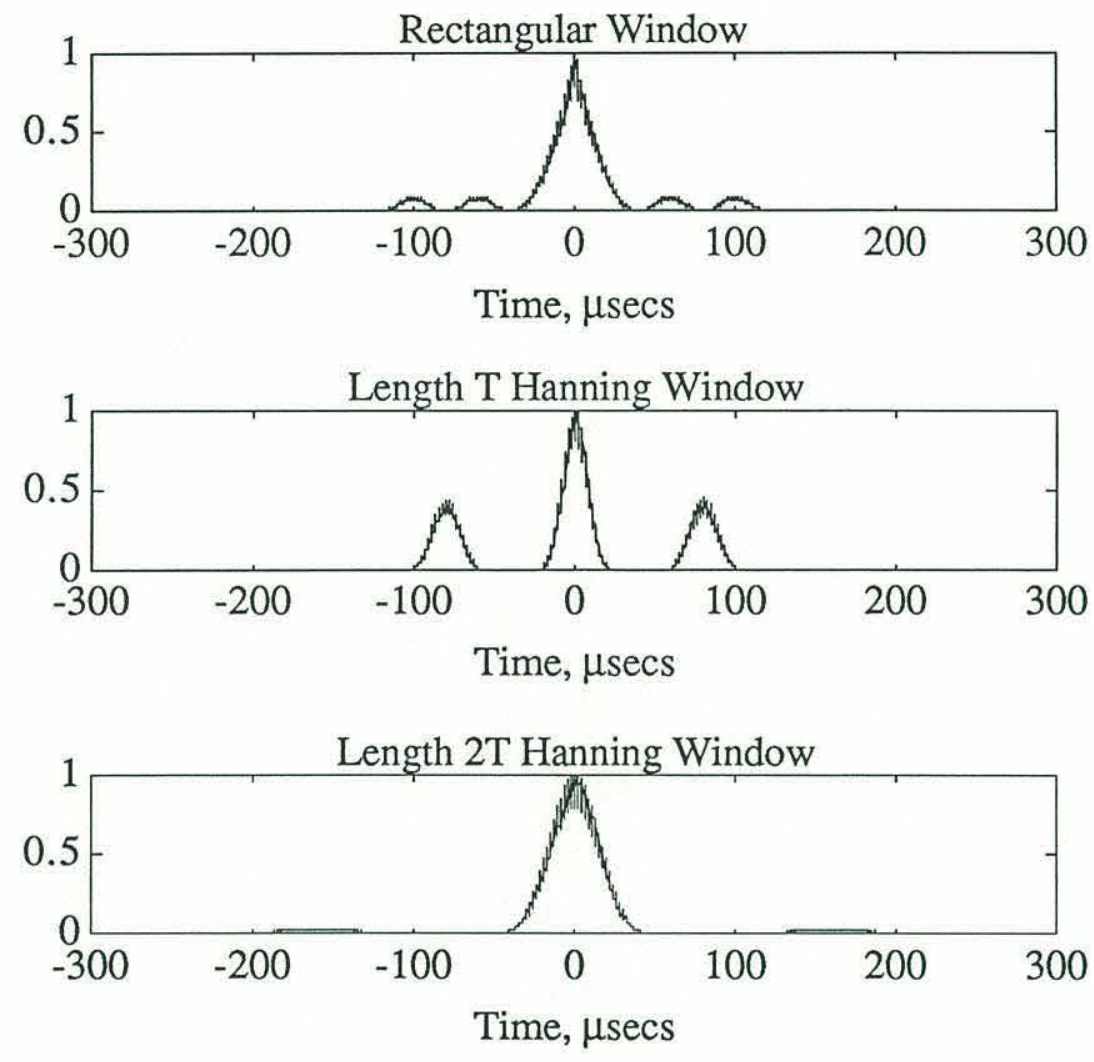

Figure 4-7: Correlation Sidelobes for Three Windows

The figure illustrates clearly that the best choice for the detection of the signal is the Hanning window of length $2 \mathrm{~T}$. The rectangular window and the length $\mathrm{T}$ Hanning window all exhibit sidelobes in the correlation function. The best implementation of the frequency-hop codes for the incoherent receiver is through the use of Hanning-windowed chips of duration $2 / \Delta f$, where $\Delta f$ is the frequency separation between chips.

One reason for the choice of the Hanning window over other windows is the ease with which it can be implemented. The incoherent receiver has a much heavier 
computational load than the coherent receiver. This will decrease the maximum frame rate achievable by the system, making real-time imaging difficult. There are some elegant algorithms that allow significant savings in the computation of discrete Fourier Transforms $[17,21]$. These algorithms result in a $\log _{2}(N)$ savings, but they can be very sensitive to windowing operations. As an option, any window can be implemented as a convolution in the frequency domain after the transform has been efficiently computed. However, the discrete Fourier Transform of the Hanning window has only three nonzero values, $-.25, .5$, and.-25 located at $-2 \pi / \mathrm{N}, 0$, and $2 \pi / \mathrm{N}$ respectively. This small number of points, coupled with the fact that the values at these points are binary fractions allows the Hanning window to be implemented quickly with only $2 \mathrm{~N}$ shift and adds. This will help offset the high computational cost of the incoherent receiver.

\subsection{Spatial Energy Distribution of the Signals}

In the introduction to the thesis, it was stated that the principle of code-division imaging was based on projecting different coded waveforms into different directions. Before discussing the details of determining a target's location, this section will more thoroughly define how the signals are projected into space.

The sixteen signals are transmitted by an array of sixteen circular transducers arranged in a four-by-four square geometry. The beam pattern of a uniformly-weighted circular disc transducer was shown in Chapter Two to be:

$$
\mathrm{B}(\alpha)=\left(\frac{2 \mathrm{~J}_{1}\{\mathrm{kR} \sin (\alpha)\}}{\mathrm{kR} \sin (\alpha)}\right)^{2}
$$

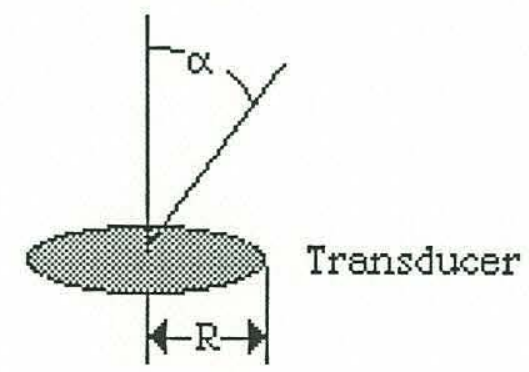


where $\mathrm{k}=2 \pi / \lambda$ is the wavenumber of the projected signal, $\mathrm{R}$ is the radius of the disc transducer , and $\mathrm{J}_{1}(\mathrm{x})$ is the first-order Bessel function of $\mathrm{x}$.

To fully describe the beam pattern for the frequency-hopped signal, it would be necessary to introduce a time-varying wavelength that results from the changes in frequency between chips. This would set up a time- and space-dependent function in that the wavelength of a signal would be a function of time and space. This complexity has been ignored in the design of the receiver. The spatial beam pattern for each transducer is assumed to be constant, and is determined from the central frequency for the bandwidth used $(900 \mathrm{kHz})$. It is recognized that the actual beam pattern at any point in time may be wider or narrower than this. These variations are not central to the principles of code-division imaging, and could be incorporated by the introduction of the time and space variation in the wavelength for each signal.

The diameter of the individual transducers was chosen such that the half-power beam width occurred at $\alpha=7.5^{\circ}$, resulting in a $15^{\circ}$ beamwidth at the $-3 \mathrm{~dB}$ points. The transducers were arranged so that the central axes were $15^{\circ}$. apart. The sonar ensonified a $60^{\circ}$ by $60^{\circ}$ aperture, composed of a four-by-four array of $15^{\circ}$ wide beams. In this geometry the beams will overlap in space, so that any point in the aperture is ensonified by a superposition of all sixteen beams.

To define a location inside the ensonified aperture, a spatial coordinate system is needed. A simple coordinate system is one which describes any point inside the ensonified aperture in terms of two angles and a range from the origin at the receiver. Figure 4-8 illustrates the coordinate system that will be used. 


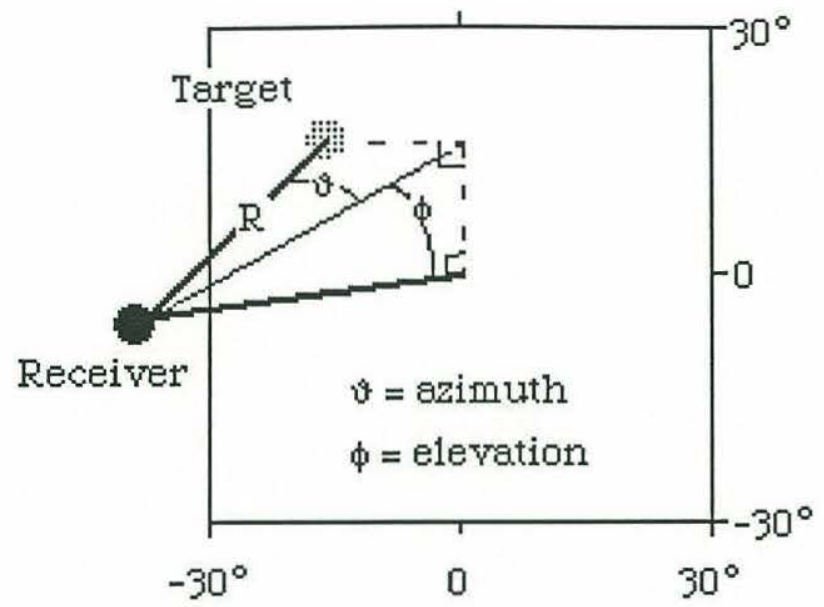

Figure 4-8: Coordinate System Convention Inside Aperture

Through a transformation of variables $\left(\sin (\alpha)=\left\{\sin ^{2}(\vartheta)+\sin ^{2}(\phi)\right\}^{1 / 2}\right)$, the beam pattern can be converted into a function of the azimuth angle ( $)$ and elevation angle $(\phi)$ :

$$
\mathrm{B}(\vartheta, \phi)=\left(\frac{2 \mathrm{~J}_{1}\left(\mathrm{kR} \sqrt{\sin ^{2} \vartheta+\sin ^{2} \phi}\right.}{\mathrm{kR} \sqrt{\sin ^{2} \vartheta+\sin ^{2} \phi}}\right)^{2}
$$

The total beam energy at any point $(\vartheta, \phi)$ (called the 'total illumination' of that point) is the superposition of the individual beam patterns ('individual illuminations'):

$$
\text { Illumination at }(\vartheta, \phi)=\mathrm{I}(\vartheta, \phi)=\sum_{\mathrm{i}=1}^{16} \mathrm{~B}\left\{\left(\vartheta-\vartheta \mathrm{i}_{\mathrm{i}}\right),\left(\phi-\phi_{\mathrm{i}}\right)\right\}
$$

where $\left(\vartheta_{\mathrm{i}}, \phi_{\mathrm{i}}\right)$ are the steering coordinates for the central axis of beam $i$. The beam pattern for the single receiving hydrophone was specially designed to be uniform throughout the ensonified aperture, and has little impact on the illumination of the points inside 
the aperture except for a slight reduction $(-6 \mathrm{~dB})$ at the corners of the $60^{\circ} \times 60^{\circ}$ field. This effect will be addressed at a later time.

Inside the ensonified aperture it is desired that the total illumination be constant for all points. Then there will be no 'dark' areas inside the aperture. This would seem to be a natural result of overlapping the beam patterns at the half-power angles and indeed the total energy fluctuates a maximum of $6 \mathrm{~dB}$, primarily at the corners of the aperture. Knowing the spatial distribution of the signal energy (illumination) for each of the coded signals is the key to estimating the spatial coordinates of a target. Figure 4-9(a and $b$ ) shows the illumination of Beam One alone, and the total illumination inside the aperture.

Using the a-priori knowledge of the spatial distribution of energy for the different coded signals, it is possible to take the first steps towards estimating the spatial coordinates of a target reflecting these signals. Ideally, the receiver should make use of all information available from the sixteen beams in order to best determine the location of a target. Section 4.4 outlines a method to estimate the reflected energy for each of the signals. 


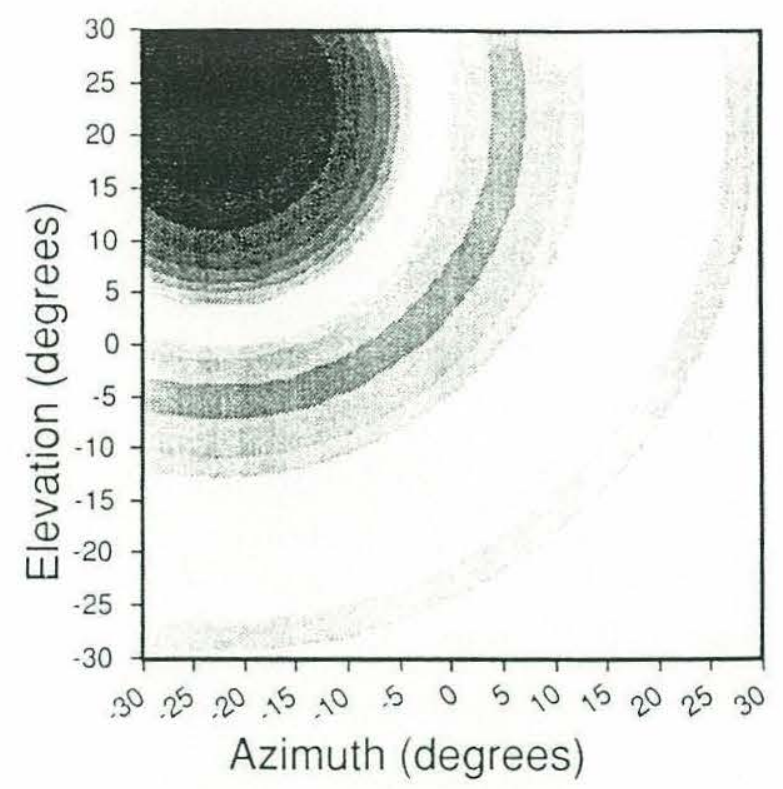

(a)

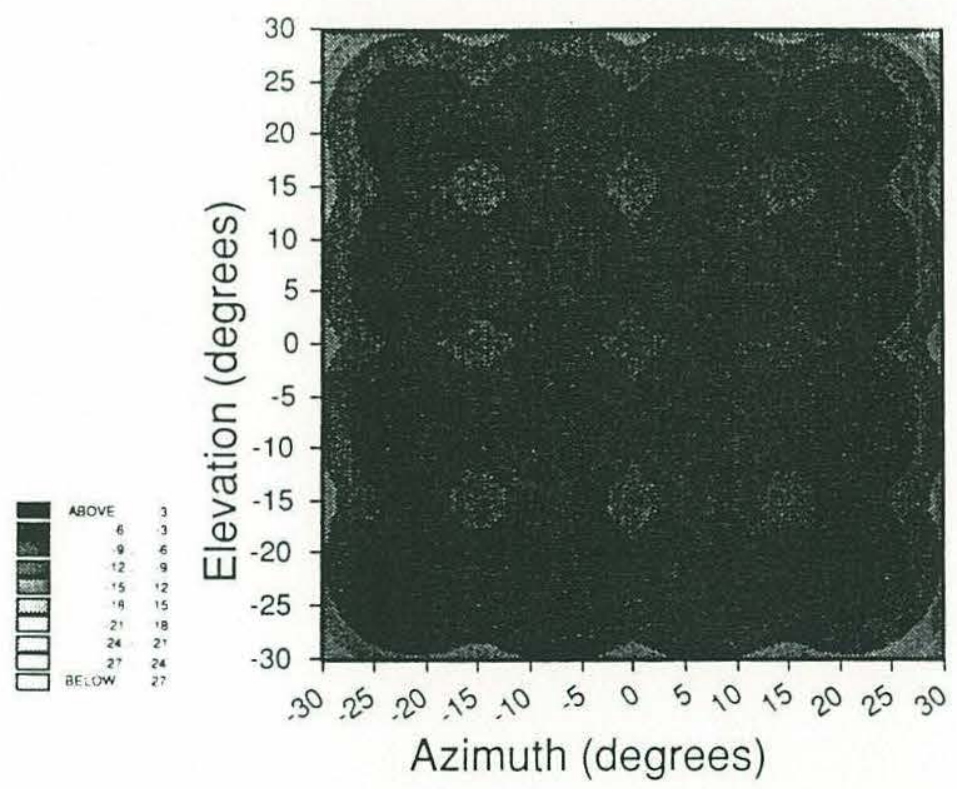

(b)

Figure 4-9: Individual Illumination for Beam One (a) and all Sixteen Beams (b), (contours in $\mathrm{dB}$ ). 


\subsection{Signal Parameter Estimation}

Given $\mathrm{N}$ targets inside the ensonified aperture of the sonar, the complete model of the reflected signal that is returned to the receiver is:

$$
r(t)=\sum_{i=1}^{M} \sum_{j=1}^{N} B_{i}\left(\vartheta_{j}, \phi_{j}\right) \widetilde{\beta}_{j} \sqrt{E_{t}} s_{i}\left(t-\tau_{j}\right)
$$

where $M=16$ is the number of coded signals projected. The illumination $B_{i}\left(\vartheta_{j}, \phi_{j}\right)$ describes the signal energy at the position of the targets. The attenuation factor $\beta_{\mathrm{j}}$ is the complex Gaussian variable discussed before. The time delay associated with a particular target, $\tau_{\mathrm{j}}$, is assumed to be the same for all signals. Given this model, it is desired to estimate the values of $\mathrm{B}_{\mathrm{i}}\left(\vartheta_{\mathrm{j}}, \phi_{\mathrm{j}}\right)$ and to make some decisions based on those estimates. In order to simplify the notation in the following discussion, a single target will be assumed.

The parameter $\beta$ was modelled in (3.2.6) as a complex Gaussian random variable with Rayleigh envelope and uniform phase. The expected value of this random variable is dependent upon $\sigma_{\beta}^{2}$ which is a function target reflectivity, range, and the medium effects, discussed in Section 4.1. The composition of the signals en ures that the energy projected is equal for all of the transmitted signals. It is reasonable to assume that for a given target, the physical processes which control these parameters are all be very similar. The magnitude of $\mathrm{B}_{\mathrm{i}}\left(\vartheta_{\mathrm{t}}, \phi_{\mathrm{t}}\right)$ is a nonrandom parameter, dependent only upon the location of the target in the beam pattern of the i-th signal. These two factors can be combined to form a new complex random variable, $\beta_{i} \mathrm{i}$ with:

$$
\mathrm{E}\left\{\left|\beta_{\mathrm{i}}{ }^{\prime}\right|\right\}=\mathrm{B}_{\mathrm{i}}\left(\vartheta_{\mathrm{t}}, \phi_{\mathrm{t}}\right) \sqrt{\frac{\pi}{2}} \sigma_{\beta} \text { and } \mathrm{E}\left\{\left|\beta_{\mathrm{i}}^{\prime}\right|^{2}\right\}=2 \mathrm{~B}_{\mathrm{i}}^{2}\left(\vartheta_{\mathrm{t}}, \phi_{\mathrm{t}}\right) \sigma_{\beta}^{2}
$$

and uniform phase as before. With these simplifications, the received signal can now be expressed from (4.4.1): 


$$
r(t)=\sum_{i=1}^{16} \beta_{i}^{i} \sqrt{E_{t}} s_{i}(t-\tau)+w(t)
$$

It is necessary to find the best estimator of the parameters $\beta_{\mathrm{i}}{ }^{\prime}$ (called the 'illumination coefficients' -- the sixteen element vector $\underline{\beta}^{\prime}=\left|\beta_{i}\right| \mid$ will be called the 'illumination vector'). The illumination coeffiecients will describe the combined effects of attenuation, reflectivity, and the beam pattern of the signal returning from the target. The relative magnitude of the parameters can be primarily attributed to the spatial variation of the beam pattern as all other factors are controlled by the physics of the problem which is the same for each parameter (In reality, a given target shape will have a reflectivity that is highly aspect dependent. In the design of a system where many unknown targets will be imaged, these effects cannot be accounted for in advance, and a point reflector will be assumed). To determine the best estimate of the illumination vector the decision variable from Section 4.2 will be used. This variable was a sufficient statistic from which to make a detection decision:

$$
\zeta_{i}^{\prime}=\Re \varepsilon\left\{\int_{t}^{t+M T} r(t) s_{i}^{*}(t) d t\right\} \quad i=1,2, \ldots, M
$$

Obviously, if $r(t)$ is of the form expressed in (4.4.2), then the variables $\zeta_{\mathrm{i}}$ are independent and normally distributed with expected value $E\left\{\beta_{i}{ }^{\prime} E_{t}\right\}$, and variance $\sigma_{n}^{2}$. The distribution of $\beta_{\mathrm{i}}$ ' has been defined as complex Gaussian with uniform phase, and Rayleigh envelope of variance $B_{i}{ }^{2}\left(\vartheta_{t}, \phi_{t}\right)(2-\pi / 2) \sigma_{\beta}{ }^{2}$. The MAP estimate of $\beta_{i}{ }^{\prime}$ is the value that maximizes the conditional probability

$$
\mathrm{p}_{\beta \mid \zeta}(\beta \mid \zeta)=\frac{\mathrm{p}_{\zeta \mid \beta}(\zeta \mid \beta) \mathrm{p}_{\beta}(\beta)}{\mathrm{p}_{\zeta}(\zeta)} \quad \text { from Bayes' Rule. }
$$

This estimate is the most likely value of $\beta_{i}{ }^{\prime}$ that would occur, given that the value $\zeta_{i}$ was observed. For this, it is necessary that 


$$
\frac{\partial \mathrm{p}_{\beta \mid \zeta}(\beta \mid \zeta)}{\partial \underline{\beta}}=0 \text { and that } \frac{\partial^{2} \mathrm{p}_{\beta \mid \zeta}(\beta \mid \zeta)}{\partial \beta^{2}}<0
$$

or equivalently:

$$
\frac{\partial\left(\ln \left(\mathrm{p}_{\zeta \mid \beta}(\zeta \mid \beta)\right)\right)}{\partial \beta}+\frac{\partial\left(\ln \left(\mathrm{p}_{\beta}(\beta)\right)\right)}{\partial \beta}=0
$$

In order to realize this in a simple, closed form, the in-phase and quadrature components will be processed separately. In the following derivation, the steps will be assumed to be carried out separately on both the in-phase and quadrature components of the demodulated signal independently, and the results will be combined at the end. The advantage of separating the problem is that the complex Gaussian variable $\beta_{i}{ }^{\prime}$ will decompose into two zero-mean, independent Gaussian variables $\mathbf{b}_{\mathbf{c}}$ (for the in phase component), and $\mathbf{b}_{\mathbf{S}}$ (for the quadrature component) with variance $\operatorname{Bi}\left(\vartheta_{t}, \phi_{t}\right) \sigma_{\beta}{ }^{2}$. The Maximum A-Posteriori rule expressed in (4.4.5) then becomes easily implemented. Substituting the specific probability density functions into (4.4.5) leads directly to the MAP estimate of $\beta^{\prime}$ :

$$
\begin{aligned}
& \frac{\partial}{\partial \mathbf{b}_{i}}\left\{\frac{-\left(\zeta_{i}-b_{i} \sqrt{E_{t}}\right)^{2}}{2 \sigma_{n}^{2}}-\frac{\mathbf{b}_{i}^{2}}{\sigma_{\beta}^{2}}\right\}=0 \\
& \mathbf{b}_{i}\left(\frac{E_{t}}{\sigma_{n}^{2}}+\frac{2}{\sigma_{\beta}^{2}}\right)=\frac{\zeta_{i} \sqrt{E_{t}}}{\sigma_{n}^{2}} \\
& \mathbf{b}_{i}\left(\zeta_{i}\right)=\frac{\zeta_{i}\left(E_{t} / \sigma_{n}^{2}\right)}{\left(E_{t} / \sigma_{n}^{2}+2 / \sigma_{\beta}^{2}\right) \sqrt{E_{t}}}
\end{aligned}
$$


This result shows that the MAP estimate of the attenuation coefficient results from applying a constant gain to the decision variable $\zeta_{\mathrm{i}}$. Since the distribution of the $\mathbf{b}_{\mathbf{i}}$ are Gaussian, this estimate is both unbiased and efficient. The error variance $\left(\sigma_{\mathrm{be}}{ }^{2}=\mathrm{E}\left\{\left(\mathrm{b}_{\mathrm{i}^{-}}\right.\right.\right.$ $\left.\left.b_{i}\right)^{2}\right\}$ for the estimate can also be obtained easily:

$$
\begin{aligned}
& \sigma_{\mathrm{be}}^{2}=\sigma_{\beta}^{2}-\frac{\mathrm{E}_{\mathrm{t}} \sigma_{\beta}^{4}}{\mathrm{E}_{\mathrm{t}} \sigma_{\beta}^{2}+\sigma_{\mathrm{n}}^{2}} \\
& =\frac{\sigma_{\beta}^{2} \sigma_{\mathrm{n}}^{2}}{\mathrm{E}_{\mathrm{t}} \sigma_{\beta}^{2}+\sigma_{\mathrm{n}}^{2}}=\frac{\sigma_{\beta}^{2}}{\frac{\mathrm{E}_{\mathrm{t}} \sigma_{\beta}^{2}}{\sigma_{\mathrm{n}}^{2}}+1}
\end{aligned}
$$

when normalized by the variance of the attenuation coefficient, (4.4.7) becomes

$$
\frac{\sigma_{\mathrm{be}}^{2}}{\sigma_{\beta}^{2}}=\left(\frac{\mathrm{E}_{\mathrm{t}} \sigma_{\beta}^{2}}{\sigma_{\mathrm{n}}^{2}}+1\right)^{-1}
$$

The only way to reduce the error variance of the estimate is to increase the signal-tonoise ratio at the receiver by increasing the transmitted power.

Once the estimates for the in-phase and quadrature components are found using the structure above, the final estimate of the attenuation coefficient can be formed by taking the sum of the squares of the two components:

$$
\beta_{i}=\left(b_{c}^{2}+b_{s}^{2}\right)^{\frac{1}{2}}
$$

where the new variable has a Rayleigh distribution function with distribution: 


$$
p_{\beta}(\beta)=\frac{\beta}{\sigma_{b e}^{2}} e^{\frac{\beta^{2}}{2 \sigma_{b e}^{2}}} U(\beta)
$$

which equals (for unit $\sigma_{\beta}^{2}$ from (4.4.7a)):

$$
\begin{aligned}
& \mathrm{p}_{\beta}(\beta)=(\sigma+1) \beta \mathrm{e}^{\frac{-\beta(1+\sigma)}{2}} \\
& \sigma=\frac{\mathrm{E}_{\mathrm{t}} \sigma_{\beta}^{2}}{\sigma_{\mathrm{n}}^{2}} \text { is the expected value of the received SNR }
\end{aligned}
$$

as previously defined (for the nonrandom detection problem) in Section 4.2. The shape of the density function for the MAP estimate of the attenuation coefficient is plotted for different values of $\sigma$ in Figure 4-10.

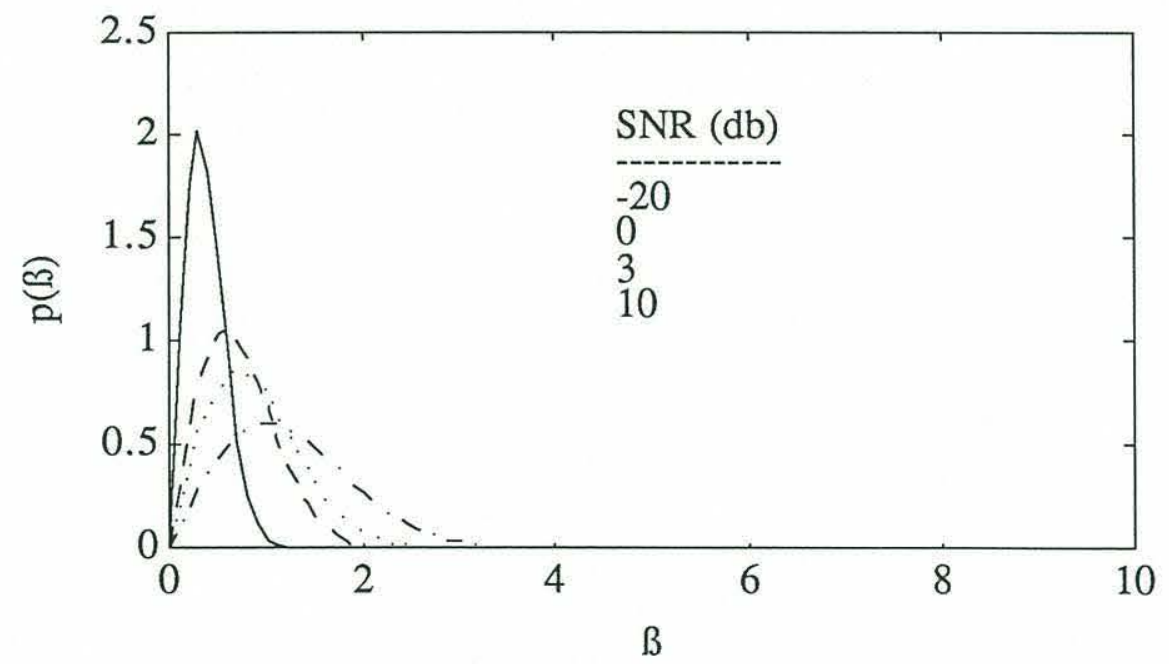

Figure 4-10: Distribution of $\beta$ as Receiver SNR Increases.

This chapter has been devoted to the design of the optimal receiver for the set of coded frequency-hopped signals. Chapter Five will develop the means whereby the estimates of the reflected energy for each of the signals in the set, $\left(\beta_{i}^{\prime} ; i=1,2, \ldots, 16\right)$ will 
be used to construct a spatial estimate of the three-dimensional position of the target field that reflected the received signal. 


\section{Chapter 5}

\section{Target Data Estimation}

The ultimate objective of the receiver designed in Chapter Four is to help determine the location of a target inside the illuminated aperture. Up to this point, the emphasis has been on obtaining the best estimate of the illumination coefficient. This estimation procedure has been carried out entirely in the time/frequency domain, taking advantage of the low cross-correlation of the signals in the time domain. In reality, the reflection coefficients are four-dimensional space/time quantities. This chapter will build on the foundation in Chapter Two, and will address the spatial dependence of the reflection coefficients.

\subsection{Spatial Correlation of the Signals Inside the Ensonified Aperture}

The illumination coefficients have been modelled as Rayleigh random variables which are independent in the time domain. The independence does not hold in the spatial domain. The spatial response function of each signal is directly related to the beam pattern associated with that signal. Since the illuminated aperture is composed of sixteen overlapping beams there is a spatial correlation between signals. The extent to which 
two illumination coefficients are spatially correlated can be qualitatively described as the amount of overlap in the beam patterns of the two signals. Stating this quantitatively, using (4.3.1) the spatial cross-correlation between two illumination coefficients is:

$$
\rho_{i, j}(\vartheta, \phi)=\left(\frac{2 J_{1}\left(k R \sqrt{\sin ^{2}\left(\vartheta-\vartheta_{i}\right)+\sin ^{2}\left(\phi-\phi_{i}\right)}\right)}{k R \sqrt{\sin ^{2}\left(\vartheta-\vartheta_{i}\right)+\sin ^{2}\left(\phi-\phi_{i}\right)}}\right)^{2}\left(\frac{2 J_{1}\left(k R \sqrt{\sin ^{2}\left(\vartheta-\vartheta_{j}\right)+\sin ^{2}\left(\phi-\phi_{j}\right)}\right)}{\left.k R \sqrt{\sin ^{2}(\vartheta-\vartheta}\right)+\sin ^{2}\left(\phi-\phi_{j}\right.}\right)^{2}
$$

Figure 5-1 shows two of the extremes for spatial cross-correlation functions. In the first figure, the cross-correlation function for two adjacent beams, $\rho_{1,2}(\vartheta, \phi)$, is shown, and in the second figure, $\rho_{1,16}(\vartheta, \phi)$ is shown for the most separated beams.
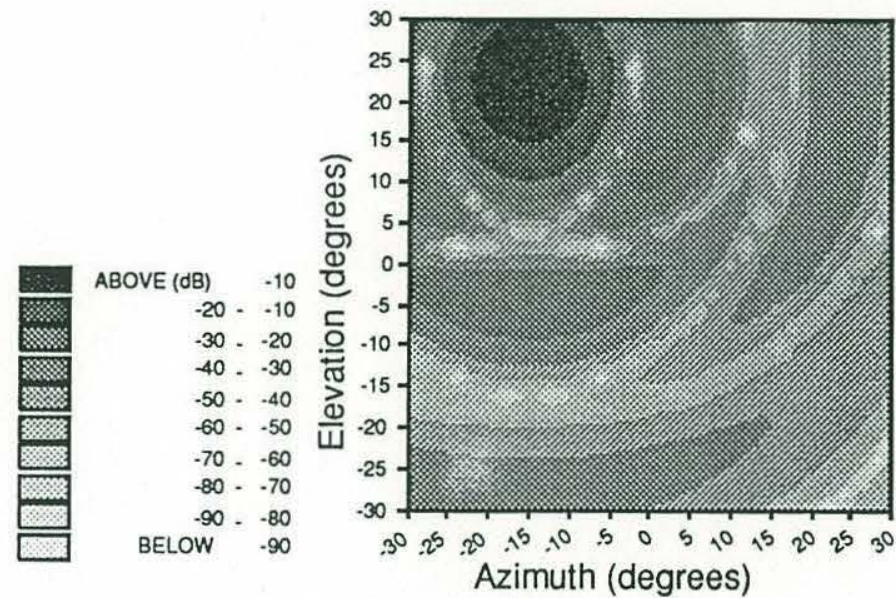

(a)

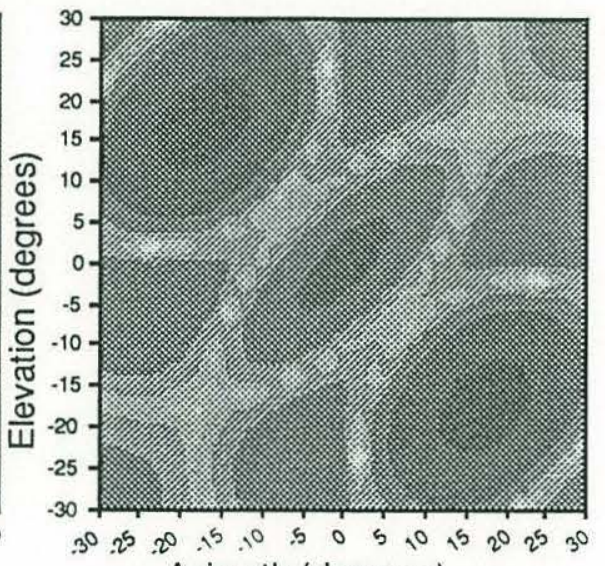

Azimuth (degrees)

(b)

Figure 5-1: Spatial Cross-Correlation Functions for (a) Illumination Coefficients 1/2, and (b) for Coefficients $1 / 16$.

If the illumination coefficients were completely decorrelated in space (i.e. their beam patterns did not overlap), then the best spatial resolution that could be attainable would be that associated with the conventional beam pattern. The cross-correlation functions between the sixteen coefficients offer a means to more fully exploit the joint information in the received signal, and obtain better resolution than is possible with 
completely disjoint beams. Since each point in the ensonified aperture is illuminated by all sixteen beams, the location of an object in the ensonified aperture can be described by the relative magnitudes of the individual illumination coefficients. For example, if the receiver detects a large value of $\beta_{12}$, and small values of all other illuminations, it follows that the target is most likely in the spatial coordinates that are ensonified most strongly by signal twelve. Similarly, if the receiver estimates $\beta_{1}$ and $\beta_{2}$ nearly equal, with all other coefficients nearly zero, then the target is most likely in the region where the cross-correlation function $\rho_{1,2}$ is maximized (see Fig. 5-1). Continuing in this way, it is possible to incorporate all sixteen elements of the illumination vector $\beta$ to determine the location of the target, decorrelating the elements of the illumination vector to obtain the best estimate of the target location. The important relation for this decorrelation is the relative magnitude of the coefficients in the vector. Hence, the exact form of the multiplicative gain used in the receiver to determine the estimate of the illumination coefficient is unimportant, as long as the relative magnitude is preserved.

The first step in determining a spatial location based on the illumination vector is to define the actual value of $\underline{\beta}$ for all points inside the ensonified aperture. This follows easily from (4.3.1 and 4.3.2):

$$
\beta_{\mathrm{i}}(\vartheta, \phi)=\mathrm{B}\left\{\left(\vartheta-\vartheta_{\mathrm{i}}\right),\left(\phi-\phi_{\mathrm{i}}\right)\right\}=\left(\frac{2 \mathrm{~J}_{1}\left(\mathrm{kR} \sqrt{\sin ^{2}\left(\vartheta-\vartheta_{\mathrm{i}}\right)+\sin ^{2}\left(\phi-\phi_{\mathrm{i}}\right)}\right)}{\mathrm{kR} \sqrt{\sin ^{2}\left(\vartheta-\vartheta_{\mathrm{i}}\right)+\sin ^{2}\left(\phi-\phi_{\mathrm{i}}\right)}}\right)^{2}
$$

or, the ideal value of the illumination coefficients is simply the magnitude of the beam pattern at that point. In practice, the aperture is discretely quantized into small spatial cells, and $\mathrm{B}_{\mathrm{i}}\left(\vartheta_{\mathrm{j}}, \phi_{\mathrm{j}}\right)$ is computed for a representative location inside each of the $j$ cells. Once the illuminated aperture has been thus quantified, the next step is to find the best way to map a received estimate of the illumination vector together with the ideal illumi- 
nation vectors for all cells in the aperture onto a map inside the aperture which shows the target's location.

\subsection{Constrained Method for Location Estimation}

Given an estimate of a received illumination vector, $\underline{\beta}$, it is desired to estimate the spatial coordinates of a target which may have generated that vector. One obvious way to do this is to conduct an exhaustive search by "steering" through all possible locations in the ensonified aperture, correlating the received illumination with the actual illumination in the steered direction. If done properly, this will result in a function of azimuth and elevation that will be maximum in the direction of the target. While an exhaustive search may sound tedious, the degree to which the aperture can be effectively quantized limits the number of cells to steer through. This idea has some similarities with coherent beamforming techniques. In the beamforming case, the elements of the complex steering vector reflect the phase difference between different elements in an array. The phase difference corresponds to the expected difference that would be generated by a plane wave incident from given direction. In the code-division system, a single receiver is used, and the steering vector consists of the pre-computed illumination vectors for the spatial cells. Using the a-priori knowledge of the total illumination inside the aperture and the incoherent receiver, an estimate of the location of the target is possible without requiring coherence over any dimension longer than a chip length.

This location estimator for the sonar, $\mathrm{H}_{\mathrm{c}}(\vartheta, \phi)$ will be constrained so that the value of the function evaluated in the true target direction will be unity (this manifests itself only in the method of normalizing the illumination vectors). The spatial correlation between the received illumination $\underline{\beta}$ and the illumination in a cell $\underline{\beta}_{j}=\underline{\beta}\left(\vartheta_{j}, \phi_{j}\right)$ can be expressed as: 


$$
\mathrm{P}_{\hat{\beta}, \beta_{j}}=\underline{\beta}^{\mathrm{t}} \underline{\beta}_{\mathrm{j}}
$$

To differentiate the correlation of illumination vectors in (5.2.1) from the cross-correlation of illumination coefficients in (5.1.1), a capital $\mathrm{P}$ will be used for the vector correlation and small $\rho$ will be used for the coefficients. For the case when $\underline{\beta}=\underline{\beta}_{j}$, the estimator should equal unity. This leads to the form of the estimator:

$$
\mathrm{H}_{C}(\vartheta, \phi)=\frac{\underline{\beta}^{\mathrm{t}} \hat{\mathbf{K}} \underline{\beta}}{\underline{\beta}^{\mathrm{t}} \mathbf{K} \underline{\beta}}
$$

where $\hat{\mathbf{K}}=\underline{\beta} \hat{\beta}^{\mathrm{t}}, \mathbf{K}=\underline{\beta}{ }^{\mathrm{t}}$, and $\underline{\beta}=\underline{\beta}\left(\vartheta_{\mathrm{j}}, \phi_{\mathrm{j}}\right)$ is the illumination vector for cell $j$.

The illumination coefficients in the aperture are spatially correlated as in (5.1.1). The expected value of the spatial correlation between cells for the constrained estimator will be the sum of the individual correlations of the elements:

$$
\begin{aligned}
& P_{m, n}\left(\vartheta_{m}, \vartheta_{n}, \phi_{m}, \phi_{n}\right)=\frac{1}{16} \underline{\beta}_{m}^{t} \underline{\beta}_{n}=\frac{1}{16} \sum_{i=1}^{16} B\left\{\left(\vartheta_{m}-\vartheta_{i}\right),\left(\phi_{m}-\phi_{i}\right)\right\} B\left\{\left(\vartheta_{n}-\vartheta_{i}\right),\left(\phi_{n}-\phi_{i}\right)\right\} \\
& =\frac{1}{16} \sum_{i=1}^{16}\left(\frac{2 J\left(k R \sqrt{\sin ^{2}\left(\vartheta_{m}-\vartheta_{i}\right)+\sin ^{2}\left(\phi_{m}-\phi_{i}\right)}\right)}{k R \sqrt{\sin ^{2}\left(\vartheta_{m}-\vartheta_{i}\right)+\sin ^{2}\left(\phi_{m}-\phi_{i}\right)}}\right)^{2}\left(\frac{2 J\left(k R \sqrt{\sin ^{2}\left(\vartheta_{n}-\vartheta_{i}\right)+\sin ^{2}\left(\phi_{n}-\phi_{i}\right)}\right)}{k R \sqrt{\sin ^{2}\left(\vartheta_{n}-\vartheta_{i}\right)+\sin ^{2}\left(\phi_{n}-\phi_{i}\right)}}\right)^{2}
\end{aligned}
$$

in this equation, the subscript $i$ denotes the steering coordinates for the central axes of the sixteen beams, and the subscripts $m$ and $n$ denote the steering coordinates for two cells in the ensonified aperture. This function defines the spatial response of the constrained estimator in cell $m$ to a point reflector located in cell $n$. In Chapter Two, the 
spatial response function for the conventional beamformer described the response of a circular aperture in direction $m$ when a unit plane wave is incident from direction $n$. Here this has been expanded to include the information from sixteen signals incident from cell $n$ The spatial response function for the constrained estimator is shown in Figure 5-2 for a plane wave incident from $\vartheta=\phi=0$, the center of the aperture.

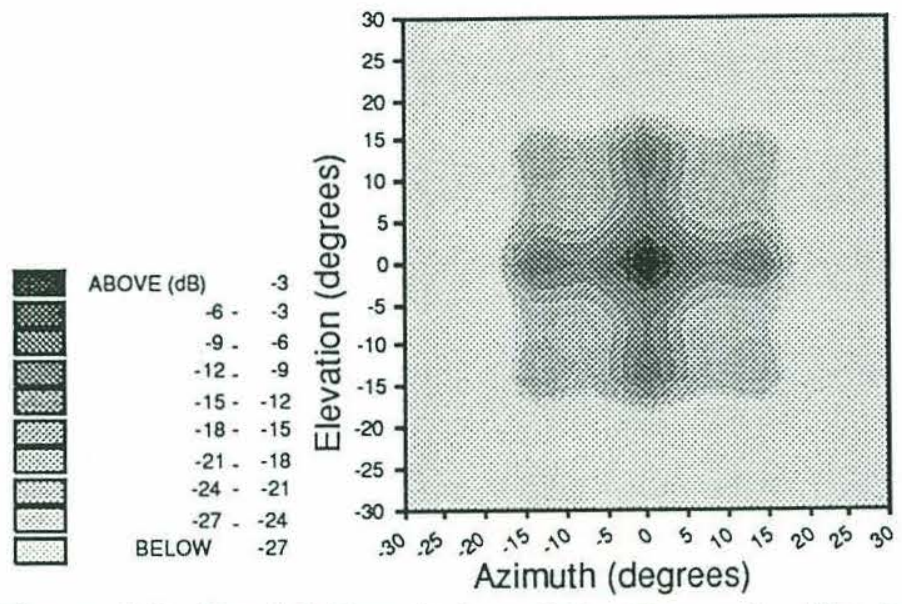

Figure 5-2: Spatial Correlation of the Constrained Estimator (target at the center of the aperture).

Figure 5-3 compares the resolution for the single transmitted beam and the constrained estimator. The constrained estimator has the same global beamwidth as the single beam, with the advantage that the constrained estimator eliminates the sidelobes (attenuated by $40 \mathrm{~dB}$ ). Additionally, the constrained estimator results in a 'focussing' of the beam at the center, allowing greater resolution than is possible with the single beam. The benefits of eliminating sidelobe ambiguity and achieving higher resolution make the constrained estimator a very suitable choice for target location estimation. 

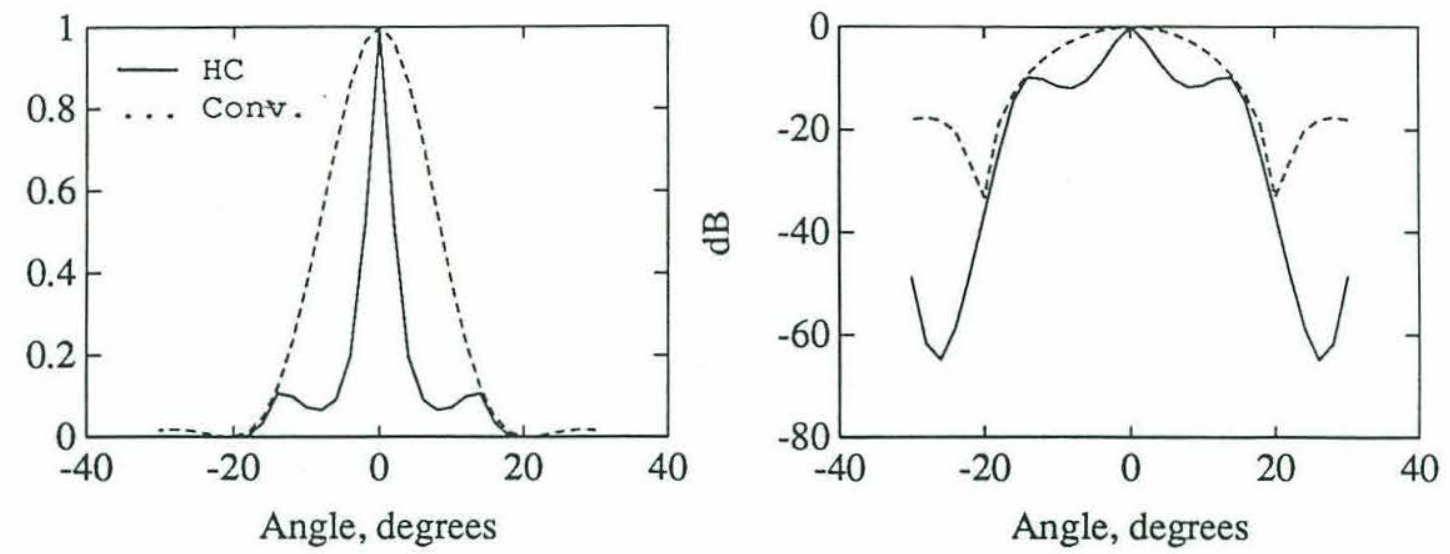

Figure 5-3: Comparison of the Resolution Capability of the Conventional Beam (dashed line) and the Constrained Estimator (solid line)

For a single target, it is generally not necessary to conduct an exhaustive search of the entire aperture. The illumination vector is usually dominated by two or three coefficients corresponding to the beams that most strongly illuminate the immediate vicinity around the target. The remaining coefficients are small because the beam energy for those signals is weak. The weak beam energy results in a lower received SNR for these coefficients, which relates to a higher variance in the estimate of these coefficients (see 4.4.7). These estimates will consequently be less reliable than the estimates of the dominant coefficients. An alternative strategy might therefore be to use only the three largest illumination coefficients, $\beta_{j}(j=1,2,3)$, and throw away the less reliable ones. The estimator could then find the largest of these three, $\beta_{\max }$, and quickly limit the search space to those cells which also have illumination vectors with the same $\beta_{\max }$. This could continue for the second largest coefficient and the third. In this way, the number of searches is reduced by at least a factor of sixteen. 


\subsection{Minimum Mean-Square Difference Estimator}

Besides spatial decorrelation with the constrained estimator, another way to estimate the position of a target would be to try to examine the difference between a received signal and an expected signal. The basic idea for this method is the same: to find the illumination vector inside the aperture that most closely approximates the estimate of the received illumination vector. If the square difference, $\Delta^{2}(\vartheta, \phi)$, between the estimated illumination and the illumination in a proposed cell is defined as:

$$
\Delta^{2}\left(\vartheta_{j}, \phi_{j}\right)=\Delta_{j}^{2}=\sum_{i=1}^{16}\left(\beta_{i}-\beta_{i}\right)^{2}
$$

evaluated for each cell $j$, then an appropriate choice for the target position is the cell associated to the $\beta$ that results in the smallest difference. One way to express this in functional form is to define a new parameter that is maximized when this difference is minimized. One functional choice is:

$$
\mathrm{H}_{\mathrm{MSD}}(\vartheta, \phi)=\frac{1}{\Delta^{2}(\vartheta, \phi)}
$$

Initially, this estimate seems unstable for $\Delta 2(\vartheta, \phi)=0$. A closer look reveals that the situation where $\Delta 2(\vartheta, \phi)=0$ corresponds to a perfect estimate of the illumination of the target. Examining (5.3.1), the expression in (5.3.2) is the difference between the estimated $\beta_{\mathrm{i}}$, a random variable, and the actual $\beta_{\mathrm{i}}$. From Chapter Four, $\beta_{\mathrm{i}}$ is the best estimate of the actual illumination for the target, and has been analyzed in detail. In fact, the expected value of the $\mathrm{H}_{\mathrm{MSD}}$ is nothing more than the inverse of the error variance, $(\sigma+1)$, for the Rayleigh distribution in (4.4.10). In real situations, an exact, noise-free measurement is not possible, as it is impossible for the distribution of the estimate to be impulsive. The MSD estimator looks at each cell in the aperture, and estimates the combined SNR for the sixteen signals radiating from that cell. 


$$
\mathrm{H}_{\mathrm{MSD}}(\vartheta, \phi)=\sum_{\mathrm{i}=1}^{16}\left(\sigma_{\mathrm{i}}+1\right)
$$

where $\sigma$ is the expected value of the received SNR, as always. If there is a point reflector in a cell, the value of the estimator will be higher than for the vacant cells. From Chapter Four, the only way to minimize the expected value of the mean square difference, $E\{\Delta(\vartheta, \phi)\}$, and thus maximize the estimator in (5.3.2) is to increase the signal to noise ratio at the receiver. Figure 5-4 illustrates the performance of the mean-square-difference receiver for different values of received signal-to-noise ratio.

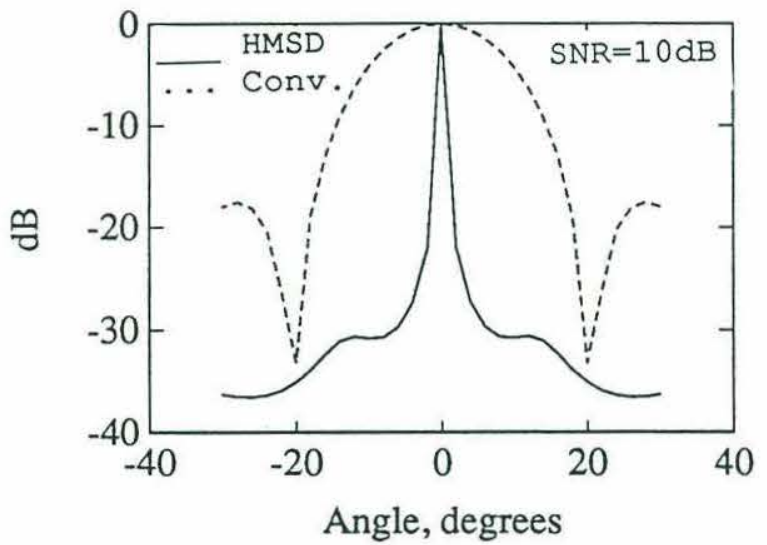

(a)

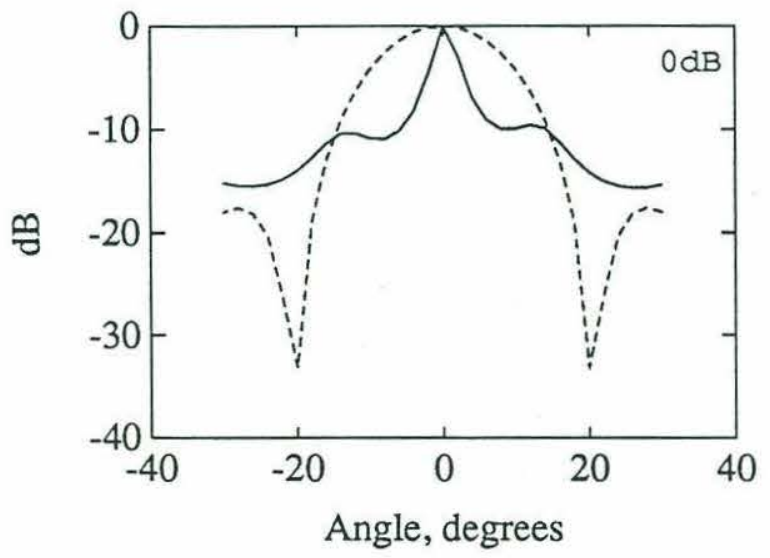

(c)

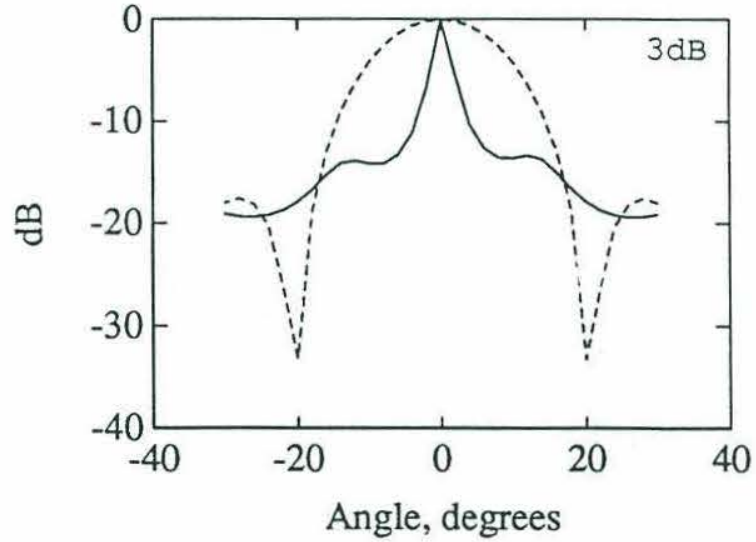

(b)

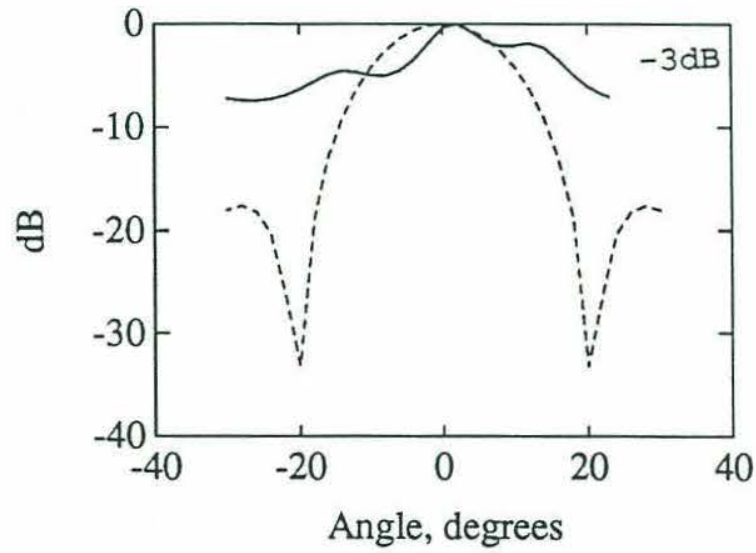

(d)

Figure 5-4: Performance of the Minimum Mean-Square Difference Estimator for $\sigma=10 \mathrm{~dB}(\mathrm{a}), 3 \mathrm{~dB}(\mathrm{~b}), 0 \mathrm{~dB}(\mathrm{c})$ and $-3 \mathrm{~dB}(\mathrm{~d})$. 
While the mean square difference estimator is capable of high-resolution performance when the signal-to-noise ratio is high, it degrades catastrophically when the SNR falls below $0 \mathrm{~dB}$. In this case, the variance in the estimate of the illumination vector is so high that the corresponding spatial estimate is either slightly peaked around the actual value (as it is in Fig. 5-4(d)), or it will peak at another set of coordinates totally unrelated to the target's location. In the case where the signal strength is low, it would be better to use the more stable constrained estimator to determine the target's location.

As a further detraction, the mean-square difference estimator does not have the potential for computational savings as does the constrained estimator. A full, exhaustive search must be performed each time the estimate is computed.

It has been determined how to best detect and estimate the important parameters associated with a reflected waveform, and to convert those parameters into a spatial estimate of the location of the object from which the signals were reflected. The spatial response and the expected performance of two spatial estimators has been examined. The next step will be to put the receiver to an experimental test in which a code-division sonar will be used to image a field of objects. 


\section{Chapter 6}

\section{Experimental Procedure and Results}

\subsection{Experimental Procedure}

In order to fully test the feasibility of the frequency-hop codes in an underwater imaging environment, a sixteen-beam sonar was constructed. Many of the specifications have been outlined already in other parts of the thesis, but they will be restated here. The sonar consists of sixteen projecting transducers and a single receiving hydrophone. The half-power beamwidth for the projectors is fifteen degrees at $900 \mathrm{kHz}$, as described in Chapter Four. The beams were arranged in a four-by-four array so as to ensonify a $60^{\circ} \times 60^{\circ}$ aperture. The half-power beamwidth of the receiving hydrophone is $57^{\circ}$. This hydrophone is mounted at the center of the four-by-four transmitting array, with it's central axis directed at the middle of the ensonified aperture. The transmitted signals for each of the projectors are individually programmable from a personal computer. For these experiments, the signals were sixteen-element frequency hop codes. The frequency hops were non-overlapping with rectangular window functions. The length of the rectangular window was shorter than that necessary to generate completely orthogonal signals. The frequency separation between adjacent chips was $25 \mathrm{kHz}$, 
which would require a $40 \mu \mathrm{sec}$ chip length. In the experiments, the chip length was only $30 \mu$ sec. Although not ideal for the incoherent receiver, the choice to use the shorter, rectangular window was driven by the small dimension of the tank in which the experiments were performed. At $17 * 30 \mu \mathrm{secs}$, the signals were $76.5 \mathrm{~cm}$ in length. The distance to the bottom of the tank was $80 \mathrm{~cm}$. In order to prevent excessive "folding over" of the transmitted signal, the chip length was necessarily shortened. The combined effects of the shortened chip length, the small tank, and the desire to image small, low reflectivity targets, made it impossible to test the incoherent receiver due to the high sidelobes, and the narrowband jamming effect (see Figure 6-1). Incoherent processing was possible only in the first phase of the experiments.

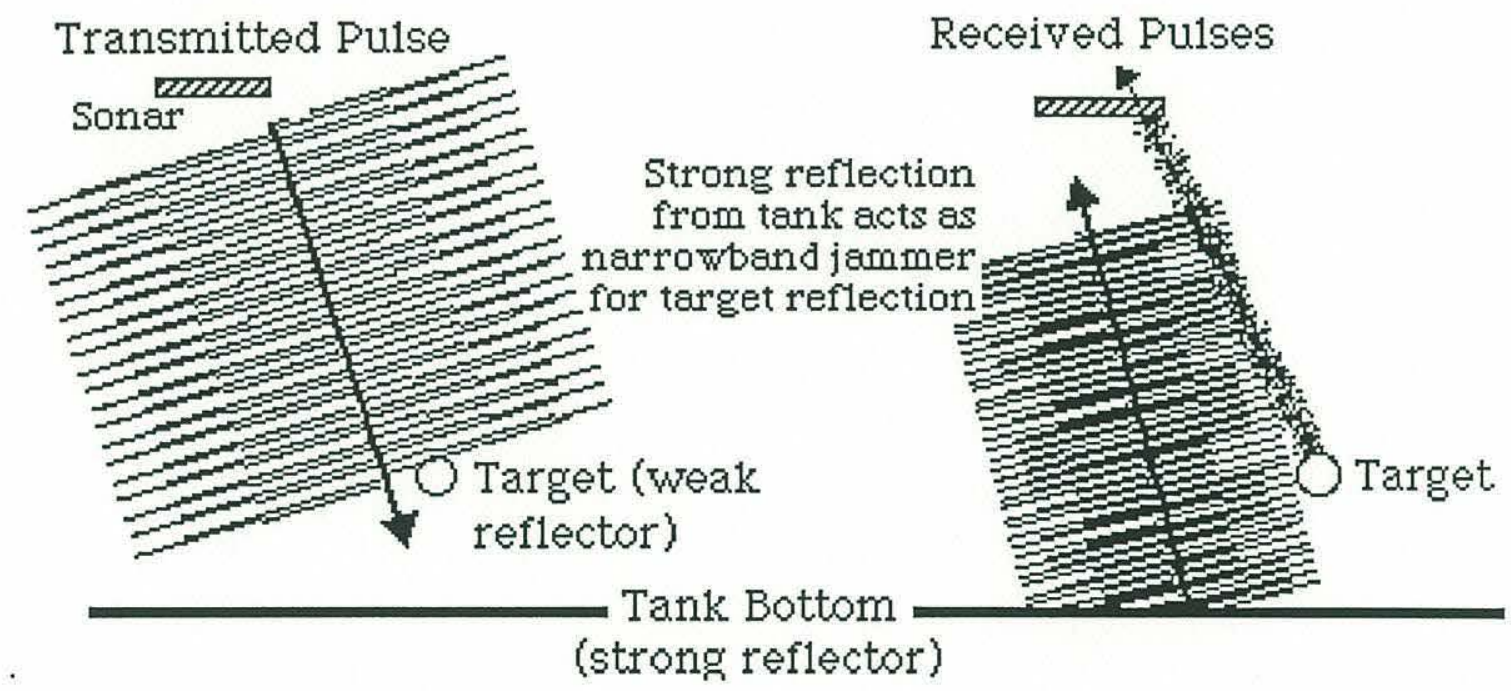

Figure 6-1: Effective Narrowband Jammer Created by Close Proximity of Tank Bottom

The reflected signal was sampled at $5 \mathrm{MHz}$, and digitized to twelve bit resolution. The transmission and reception sequence is controlled from the same personal computer that programs the signals. In the present configuration, all signal processing is done off-line on a different computer. The sonar was used in a number of experiments designed to simulate several different target and medium environments. The sonar was mounted in a water-filled tank (water temperature was approximately $20^{\circ} \mathrm{C}$ ), and targets 
were placed at various positions in the ensonified aperture. The experimental setup is illustrated in Figure 6-2.

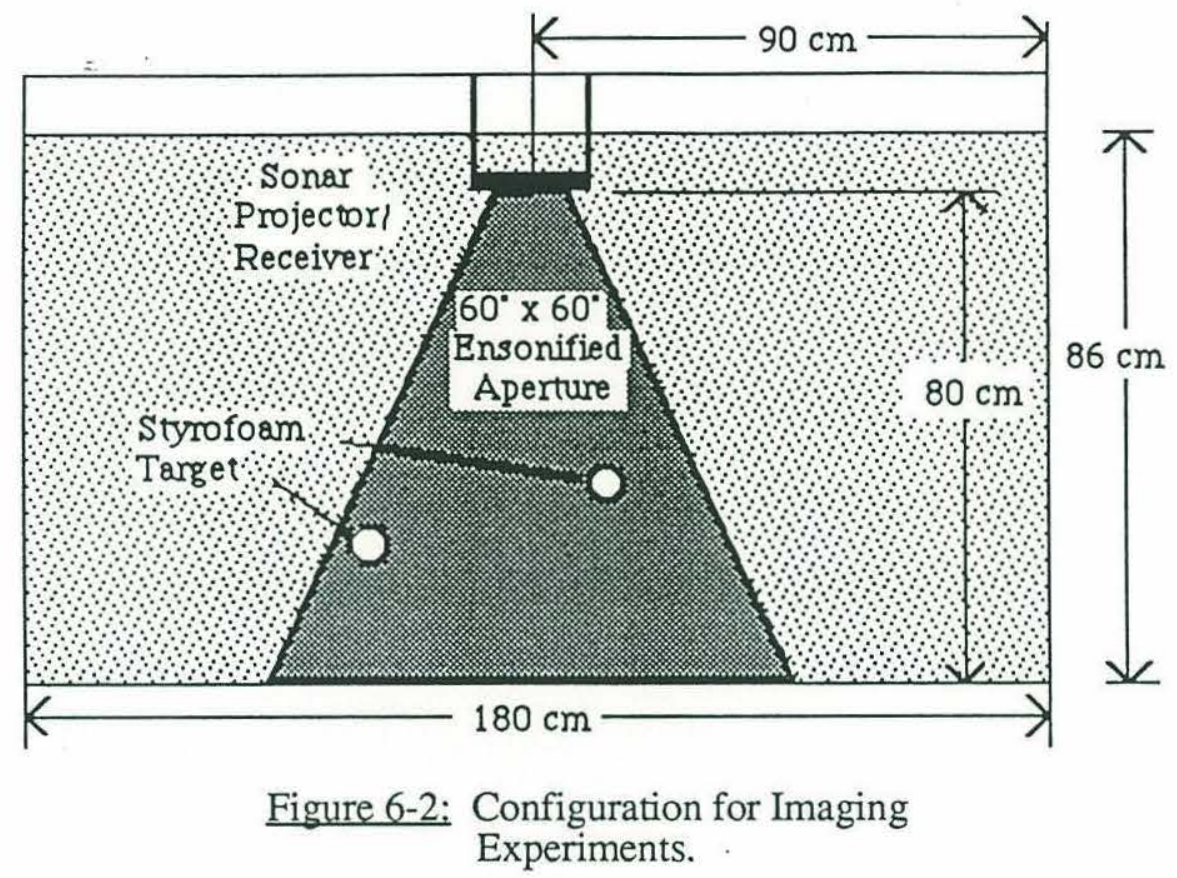

The experiments consisted of four phases: Phase One consisted of reflecting the sixteen signals one at a time off the bottom of the tank, and recording each them independently. The bottom of the tank was a strong reflector, and the received signal to noise ratio was consequently very high $(16 \mathrm{~dB})$. This phase simulated a single strong target in one of the beams, to test the operation of the receiver under near ideal conditions.

Phase Two consisted of placing a single target in the sonar's field of view. The targets were all styrofoam spheres with a $5 \mathrm{~cm}$ diameter. Styrofoam was selected for its excellent acoustic reflectivity due to the large impedence mismatch. In addition, the rough surface prevented a single, specular reflection that might not return to the receiver. Although the target geometry was known a-priori, this information was not used in the processing of the signals. The aspect dependence of the target strength for a rough sphere can result in wide variation in the reflected energy [19] from the different beams. The object of the experiments was to simulate the detection of an unknown target, so 
specific target strength calculations were not incorporated. The position of the target was varied while a number of transmit/receive sequences were performed. Each position of the target was recorded and later compared to the predicted location. This tested the performance of the receiver for a single, weak target. In addition, since the targets were suspended in the water column between the sonar and the bottom, the ability of the sonar to detect a weak target (the styrofoam target) while being "jammed" by a stronger target (the bottom of the tank) was also tested.

Phase Three expanded on Phase Two in that another styrofoam target was added. The two targets were moved around inside the ensonified aperture at varying range and angular separation. As before, the positions of the targets were recorded, for later comparison with the results of the receiver. Range separation for the two targets varied from zero to fifteen $\mathrm{cm}$. Azimuthal separation varied from $0^{\circ}$ to $50^{\circ}$. This phase of experiments was designed to test the ability of the system to separate two distinct targets within the aperture. As in Phase Two, the presence of the bottom provided a jamming signal.

The final phase, Phase Four, consisted of imaging a single target in a rapidly fluctuating medium. Random circulating currents were induced between the target and the sonar with a high-speed propeller. The propeller was rotated at the maximum speed possible while avoiding cavitation (approximately $200 \mathrm{rpm}$ ). The propeller was left running during the imaging process. The currents induced were rapidly changing in a distance on the order of the wavelength used, and the medium was perturbed for a distance of several hundred wavelengths. The goal of this phase was to simulate a current field (that might result from sensor motion and ocean currents) that would distort the phase of the signals and test the relative performance of the coherent and incoherent receivers for the signals in a fluctuating medium.

The preliminary component testing and fabrication took place at the Woods Hole Oceanographic Institution, Woods Hole, Massachusetts. The experiments were con- 
ducted during January 1989 at the Marine Physical Lab at the Scripps Institution of Oceanography in San Diego, California. The data was recorded on computer tape, and the processing was done off-line at the Research Laboratory of Electronics at MIT in Cambridge, Massachusetts.

\subsection{Experimental Results}

The results for all phases of the experiments demonstrate the feasibility of codedivision imaging. In each phase, the returned signal reflected from the targets was successfully detected. The experimental results will be presented in two stages, following the development in the previous chapters. First, the results of detecting the received frequency-hop codes will be presented for all phases, showing the degree of signal-to-signal rejection that was achieved. Next, the performance of each of the placement algorithms will be shown for a single target in a variety of positions inside the aperture.

\subsubsection{Signal Detection}

As expected, Phase One yielded the most definitive results due to the higher signal to noise level at the receiver $(16 \mathrm{~dB})$. Figure 6-3(a) shows the coherent detection of signal two $\left(s_{2}\right)$ when correlated against all sixteen signals. As can be seen, the correlation peak is narrow, and code-to-code rejection was nearly complete. As a consequence of the high SNR, the incoherent receiver was also tested. Despite the unfavorable characteristics of the signal construction, without the jamming effect the incoherent receiver was able to perform as well, as shown in Fig. 6-3(b). 


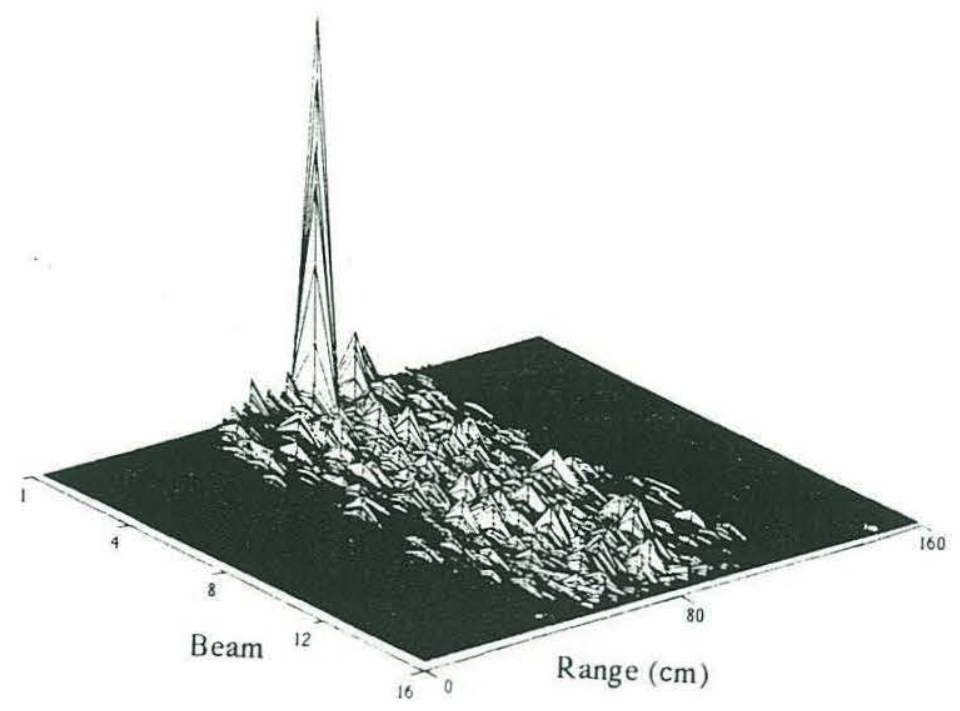

(a)

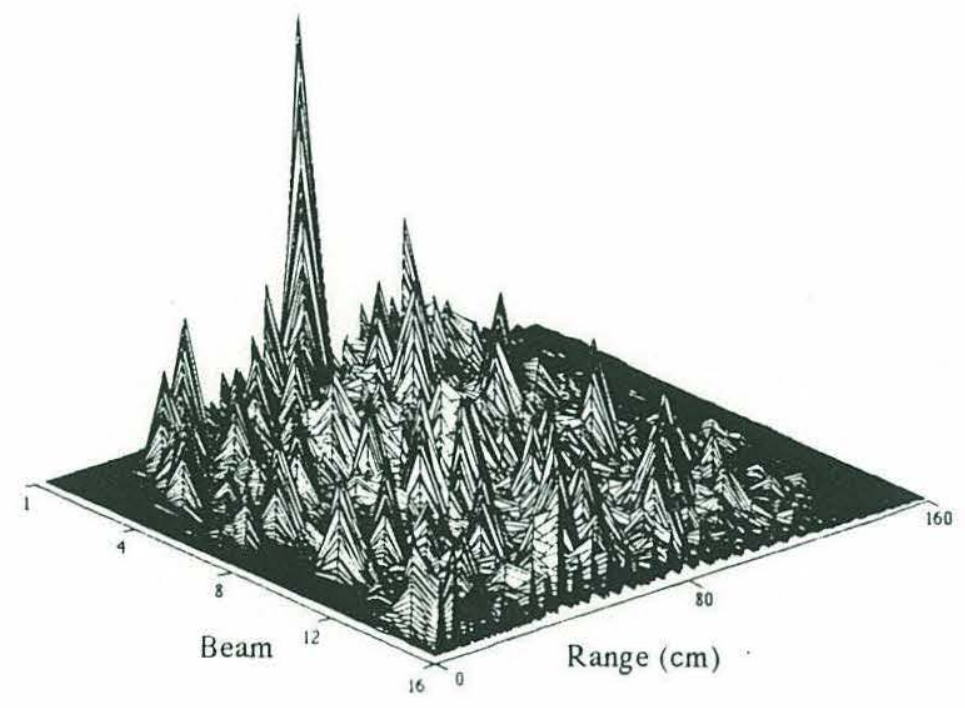

(b)

Figure 6-3: Detection of Target in Beam Two.

Coherent (a) and Incoherent (b) 'Phase One)

The introduction of the styrofoam targets in the latter three phases reduced the signal-to-noise ratio to 3-4 dB. The conditions in the tank were very calm, reducing the possibility of phase-perturbation by the medium. The only phase distortion expected was a constant offset due to reflection by the target. The importance of the internal phase structure is seen in the outstanding performance of the coherent detector. Figure 6-4 illustrates the detection of a target present in Beam 16 at a range of $32 \mathrm{~cm}$. 


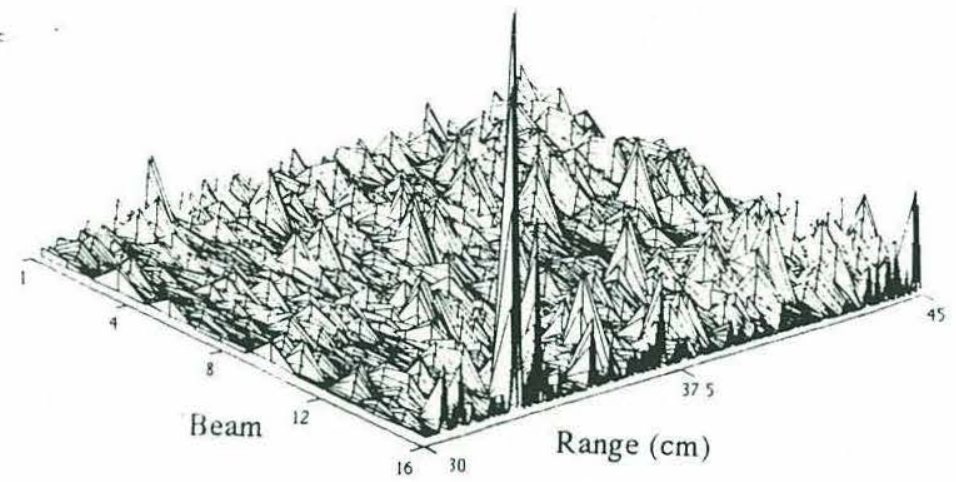

Figure 6-4: Coherent Detection of a Single Target in Beam 16 (Phase Two).

The conditions for Phase Three were identical to Phase Two except that a second target was placed in the ensonified aperture. The coherent detector was able to detect both targets simultaneously, as designed. This result demonstrates the suitability of code-division imaging for the simultaneous detection of closely spaced multiple targets. Figure 6-5 shows the coherent detection plane for two targets, one located in Beam 11 and one located in Beam 15. The azimuthal separation of these targets was ten degrees, demonstrating the potential angular resolution of the system.

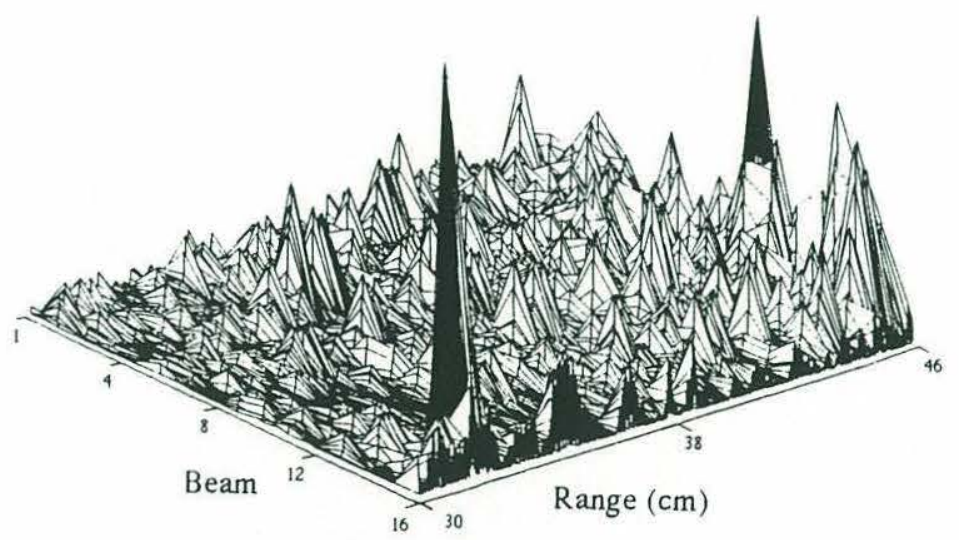

Figure 6-5: Coherent Detection of Two Targets, one each in Beam 11 and 15 (Phase Three) 
Phase Four was designed to introduce rapid phase oscillations which would degrade the performance of the coherent receiver to the point that it would not achieve detection. The phase distortion resulted in a correlation peak that was four to five times as wide as in the previous experiments (see Figure 6-6) due to spreading that nok place as the signal passed through the fluctuating currents. However, there was enough internal structure to allow coherent detection of the signal.

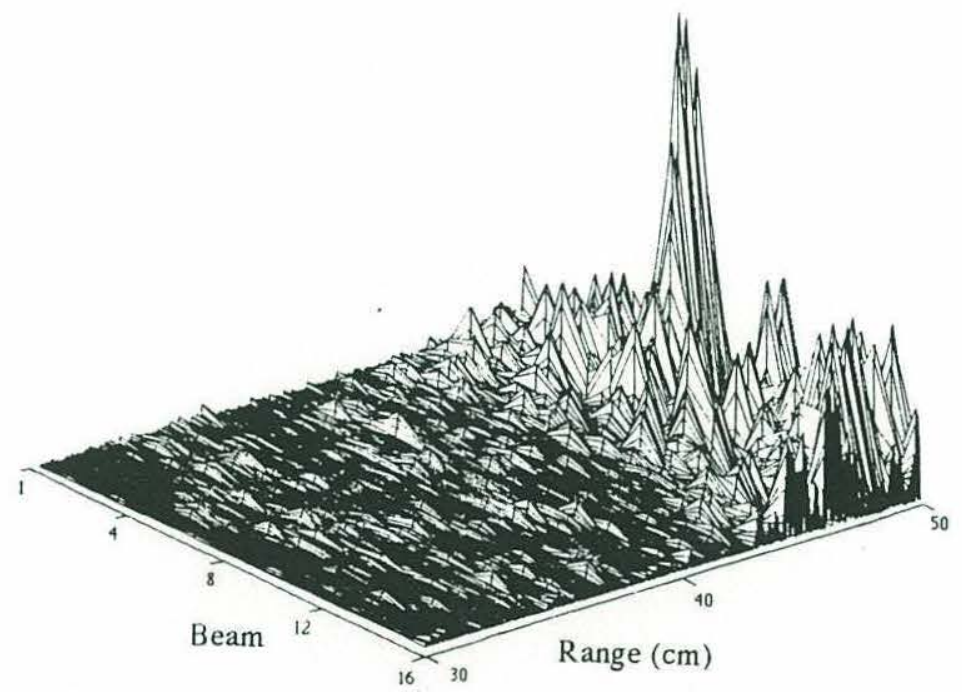

Figure 6-6: Coherent Detection of a Target in Beam 8 Imaged Through a Fluctuating Medium (Phase Four)

\subsubsection{Target Location and Image Construction}

The results presented here are planes which show the estimated azimuth and elevation of the target. The predicted location matched the actual location in each case. The five $\mathrm{cm}$ diameter of the sphere spans nine degrees in angle at the range of the target. In the instances where there were multiple targets, it was artificially assumed that they were at the same range, to examine the ability of the imaging algorithms to resolve two closely spaced targets. If range separation was factored into the problem, resolving the two targets is easier since the algorithm completely separates the two illumination vectors. 
Artificially constraining the two targets to be at the same range imposes the most rigorous test.

The figures show the result of processing data from Phase Two of the experiments, tracking a single styrofoam target through different portions of the aperture. Figure 6-7 shows the result for the target located in the middle of the aperture. The constrained estimator is shown in Fig 6-7(a), and the mean-square difference estimator is shown in Fig. 6-7(b). In this particular case, the target was located in the very center of Beam Six. Consequently, there was little energy reflected from the other beams, and the illumination vector was dominated by element six. The lack of information from the other signals resulted in a resolution that is little better than the conventional beam pattern for both cases.

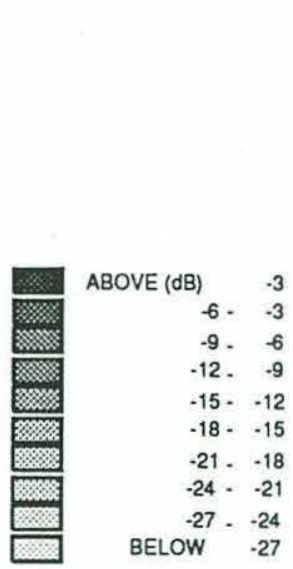

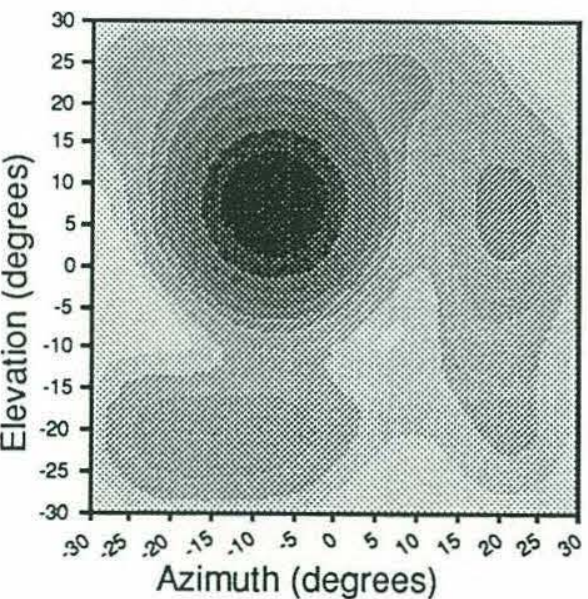

(a)

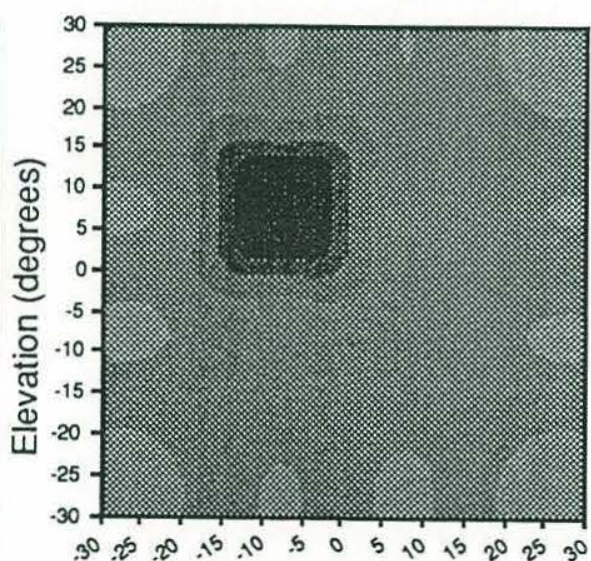

Azimuth (degrees)

(b)

Figure 6-7: Detection of Target in Beam Six, (a) Constrained Estimator, (b) Mean-Square Difference Estimator.

Next, the target was placed in the corner of the aperture, where the total illumination was the lowest. This test was designed to observe the resolution of the estimators in these locations. Figure 6-8 shows the estimated and actual positions of the target at the lower right corner of the aperture. As predicted, the resolution of the two estima- 
tors was wider than at the center of the aperture. As the target was moved along the lower edge towards the center of the lower edge, the resolution immediately improved. As information from the other beams increased (primarily from beam fifteen), the mean square estimator was able to locate the target with very high resolution. Figure 6-9 shows the improvement in resolution for both estimators.

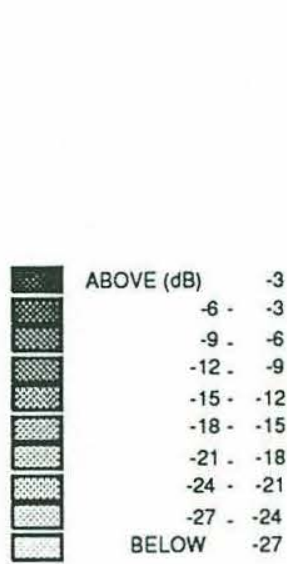

(a)

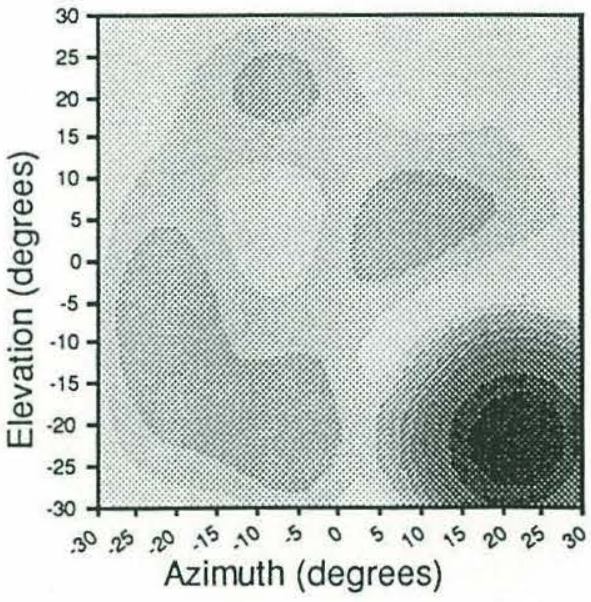

a)

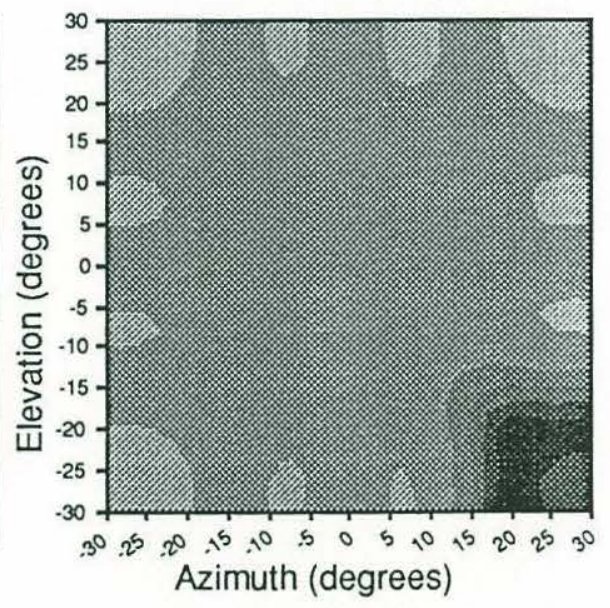

(b)

Figure 6-8: Target Detection in Lower-Right Corner of the Aperture (a) Constrained Estimate and (b) Mean-Square Difference Estimate.

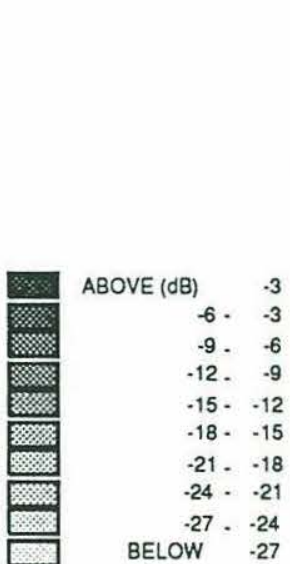

(a)

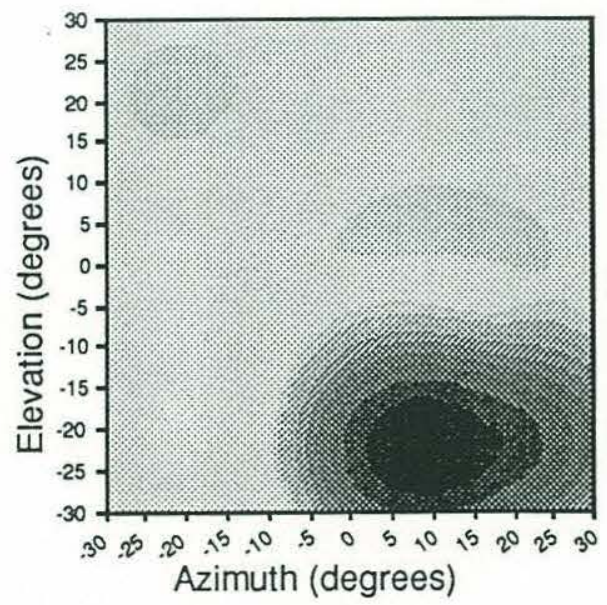

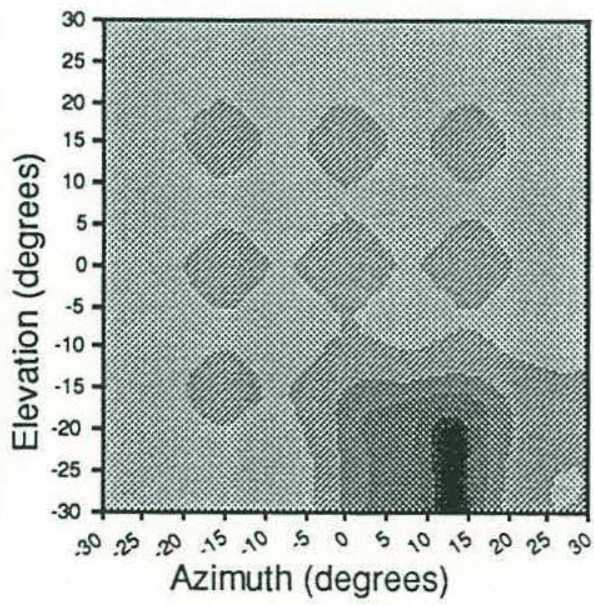

(b)

Figure 6-9: Improved Resolution Obtained as Target Moves into Aperture. (a) Constrained and (b) Mean-Square Difference. 
The last images to be obtained were for multiple targets from Phase Three.

The detection data from Figure 6-5 was used to see if the targets could be separately resolved. Although the targets differed in range by approximately $20 \mathrm{~cm}$, the illumination vectors for the two cases were artificially combined to simulate two targets with the exact same range. As discussed, this artificiality tests the estimators more rigorously.

Figure 6-10 shows the actual resolution obtained from the range-separated targets while Figure 6-11 shows the resolution of the targets simulated to have the same range.
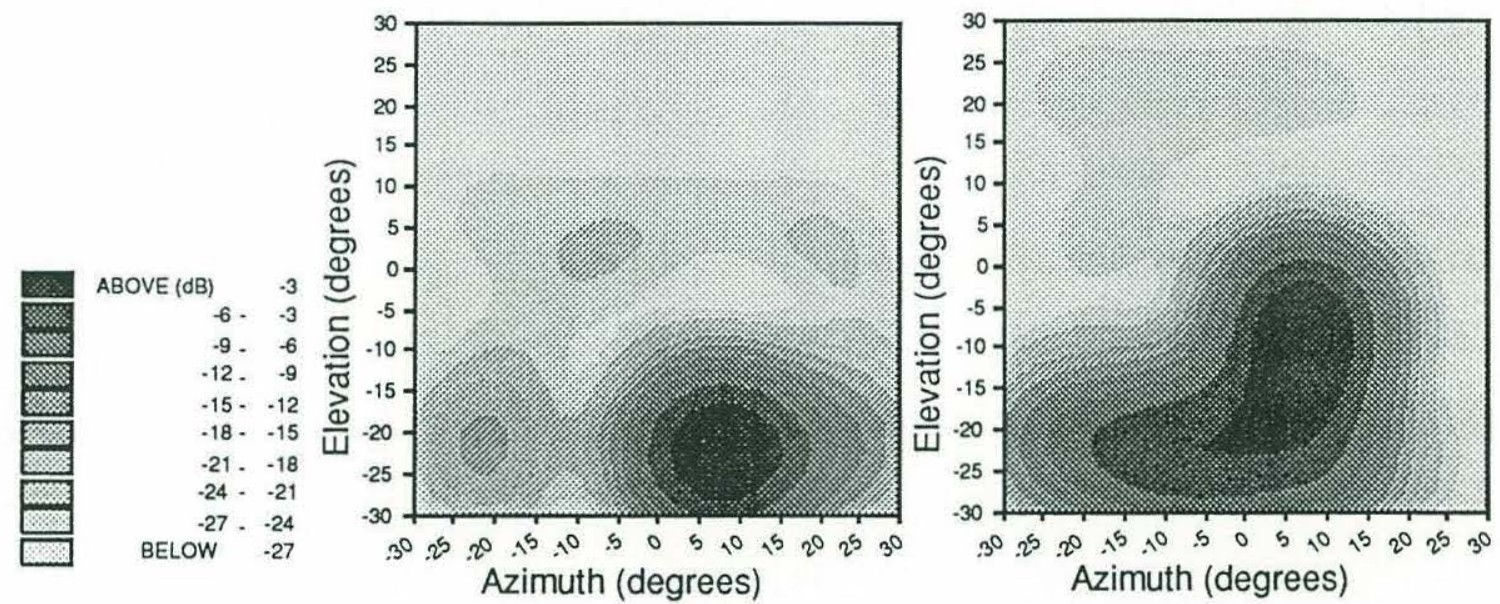

(a)
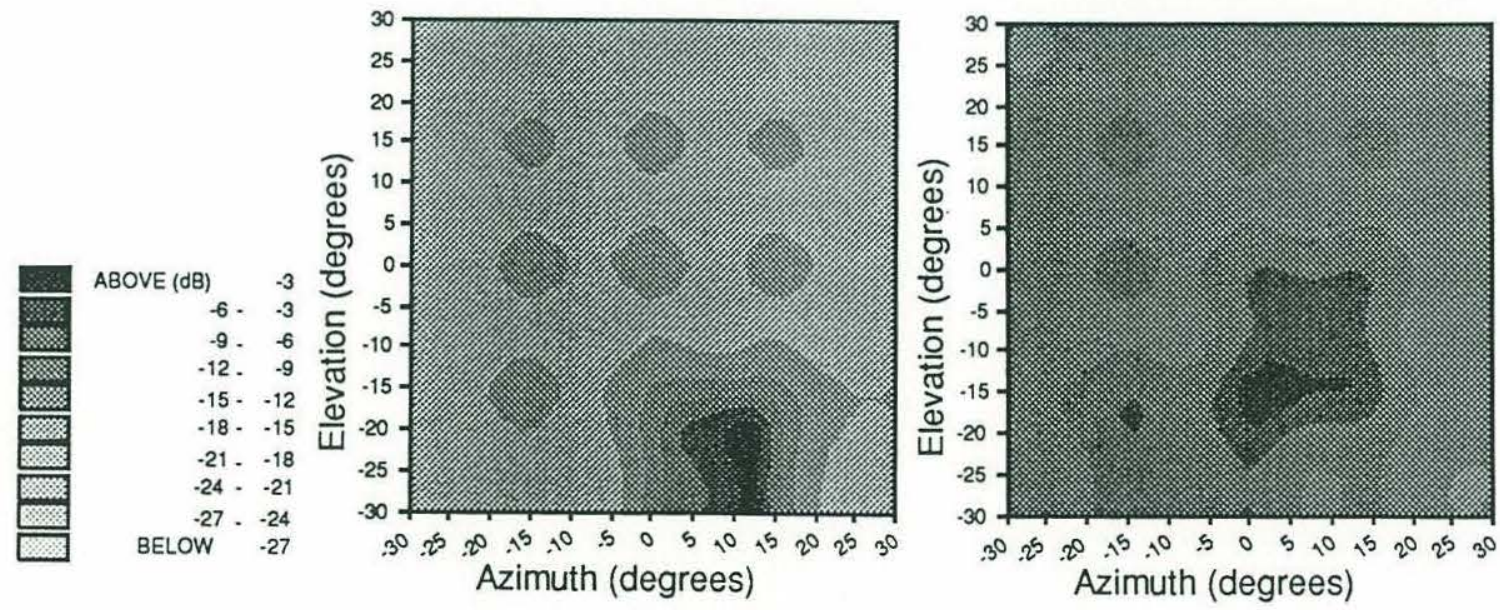

(b)

Figure 6-10: Detection of Range Separated Targets.

(a) Constrained and (b) Mean-Square Difference 


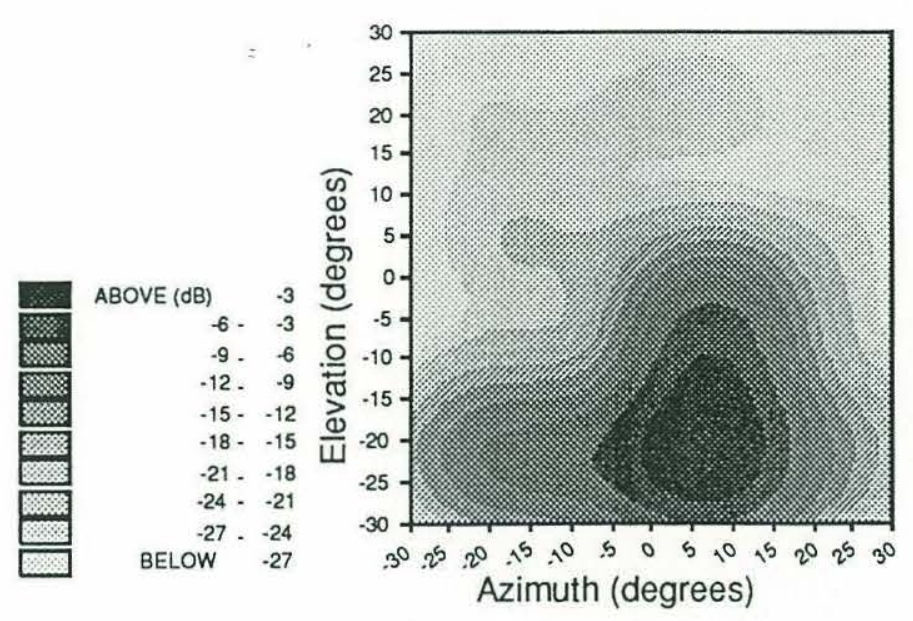

(a)

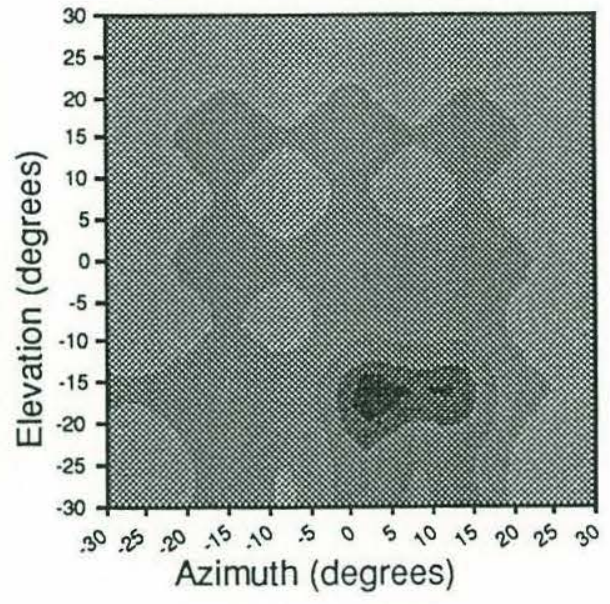

(b)

Figure 6-11: Detection of Two Targets Simulated to have the Same Range (a) Constrained and (b) Mean-square Difference.

Bearing separation was possible when the range difference was factored into the problem, but neither of the spatial estimators was able to separate the targets when the same range was simulated. This would have been remarkable in light of the fact that the diameter styrofoam targets spans nine degrees at the range of 32 centimeters. 


\section{Chapter 7}

\section{Conclusions and Further Work}

\subsection{Capabilities and Limitations}

Throughout the preceding chapters, the goal has been to introduce a new sonar to image a three-dimensional field. Perhaps the best way to summarize the thesis results is to return to the research questions posed in the introduction:

The best way to implement the coded signals is in the form of windowed frequency hop codes. The windowing operation carried out on the frequency chips effects the performance of the incoherent receiver. A Hanning window was shown to be effective in reducing the sidelobes of the incoherent detector if the chip length was made twice as long as the equivalent rectangular window. The Hanning window is easily and quickly implemented in the frequency domain as a three-point convolution, increasing the speed of the processor.

The frequency-hop codes proposed by Jaffe in $[7,8]$ were shown to be suitable for code division imaging through an examination of their range-doppler ambiguity functions. The signals did show limited capability in resolving moving targets. Specifically, the coded signals exhibit a global ambiguity in the presence of dopplershifted returns. To overcome this limitation requires either that the sonar be limited to situations where the targets do not exhibit an excessive range-rate, or that the signals be modified to use a wider bandwidth. 
The best receiver for the codes was found using basic principles of communication theory. Two spatial location estimators were presented. The spatial estimators were shown to exhibit better resolution than is capable for the conventional single beam by exploiting the spatial correlation between different projected beams inside the aperture. This relied heavily on the ability to separate the individual signal returns from the ensemble reflected waveform in the time domain.

For the case of phase perturbation, the incoherent receiver for the frequency hop signals was shown to be effective in estimating the position of a target if: 1) The received signal-to-noise ratio was sufficient to overcome the incoherent combining loss, and 2) There wasn't a wide variation in the reflectivity of close (defined as within a signal length) scatterers. In the former case, the incoherent receiver is unable to estimate the position of the target due to the wide variance associated with the estimate of the illumination vector. In the latter case, the presence of a strong, narrowband jamming effect resulted from the signal reflected off of the strong target "drowning out" the signal from the weak target. Additionally, the incoherent receiver is computationally burdensome, and would consequently slow the maximum frame rate of the system. Still, the capability to image in perturbative environments is one of the most attractive features of this system.

In the acoustic channel, experiments showed the coherent estimator to be quite capable, even in instances when phase perturbation was deliberately introduced. The internal phase structure of the signal was preserved enough to allow reliable detection. Additionally, the coherent receiver is not as susceptible to narrow band jamming. This property allows for better performance in situations where multipath propagation is probable, or where the field to be imaged has a spatially variant reflectivity.

In light of existing capabilities, the code-division sonar offers a means previously unavailable to image in three-dimensions in highly disruptive environments. 
As stated in the introduction, the existing technology, while very sophisticated, was unusable in many applications due to rapid phase perturbations, rapidly changing field of view, or a lack of multi-element sonar arrays. Code-division multiple-beam imaging has offered a potential solution to fill some of those gaps.

\subsection{Further Work}

In the future, the emphasis for further development should concentrate on reducing the time required to estimate a target's location. The spatial location estimators presented in this thesis have much potential for improvement, both in speed and in resolution. The exhaustive search methods outlined in Chapter Five could be made more efficient to increase the speed of the process. The use of task-specific hardware would implement the detection process much faster than the off-line computer. The implementation of iterative algorithms to aid in separating close targets would seem appropriate.

Experimentally, the incoherent receiver was not tested. One of the most significant contributions that the code-division sonar makes is the ability to operate in phaseperturbative environments. This could be verified experimentally by conducting a series of experiments in a more spacious environment which would prevent a strong reflection from a boundary jamming the returns from the experimental targets.

The development of code-division imaging has just begun. The initial theoretical and experimental results hold much promise. Given the potential gains it offers, further improvements in this field should be the subject of close examination. 


\section{References}

1. Cooley, J.W., and J.W Tukey, "An Algorithm for the Machine Calculation of Complex Fourier Series," Math. Computation 9, 1965, pp. 297-301.

2. Capon, J., "High-resolution Frequency-wavenumber Spectrum Analysis," Proc IEEE 57, Aug 1969, pp. 1408-1418.

3. Burg, J.P., "Maximum Entropy Spectral Analysis," Proc. 37th Meeting Society of Exploration Geophysicists (SEG), Oklahoma City, OK., Oct 31, 1967.

4. Johnson, D.H., and S.R. DeGraaf, "Improving the Resolution of Bearing in Passive Sonar Arrays by Eigenvalue Analysis," IEEE Trans. ASSP 30, \#4, pp. 638-647.

5. - -- "The Application of Spectral Estimation Methods to Bearing Estimation Problems," Proc IEEE 70, 1982, pp. 1018-1028.

6. Baggeroer, A.B., and W.A. Kuperman and H. Schmidt, "Matched Field Processing: Source localization in correlated noise as an optimum parameter estimation problem," J. Acoustical Soc. of Am. 83, February 1988. pp. 571-587.

7. Jaffe, J.S. and P.M. Cassereau, "Multibeam Imaging Using Spatially Variant Insonification," J. Acoustical Soc. Am., 83, April 1988, pp.1458-1464.

8. Jaffe, J.S., D. Glassbrenner, and P.M. Cassereau, "Incoherent Coding Techniques and Performance Characterization for Multibeam Sonar Systems, "IEEE Proc. ICASSP88, New York, NY, pp. 2709-2712.

9. Baggeroer, A.B., Space/Time Random Processes and Optimum Array Processing. Naval Undersea Center Report TP 506, Naval Undersea Center, San Diego, CA., 1976.

10. Reed, I.S. and G. Solomon, "Polynomial Codes Over Certain Finite Fields," J. of the Soc. for Ind. and Applied Math. (SIAM) 8, 1960, pp 300-304.

11. Proakis,J.G., Digital Communications, McGraw-Hill, New York, 1983.

12. Van Trees, H.L., Detection, Estimation, and Modulation Theory, Part III. Wiley, New York, 1971.

13. Rihaczek, A.W., Principles of High Resolution Radar, McGraw-Hill, New York, 1969.

14. Fisher, F.H. and V.P. Simmons, "Sound Absorption in Sea Water," J. Acoustical Soc. Am. 62, 1977, p. 558. 
15. Oppenheim, A.V. and Schafer, R.W., Discrete-Time Signal Processing, Prentice Hall, Englewood Cliffs, N.J., 1989.

16. Harris, F.J., "On the Use of Windows for Harmonic Analysis with the Discrete Fourier Transform," Proc IEEE 66, 1978.

17. Covell, M.M. "An Algorithm Design Environment for Signal Processing" Ph.D. Thesis, Massachusetts Institute of Technology, Aug 1989

18. Urick, R.J., Sound Propagation in the Sea, Peninsula Publishing, Los Altos, Ca. 1982.

19. Morse, P.M. and K.U. Ingard, Theoretical Acoustics, McGraw-Hill, New York, 1968.

20. Rice, "Envelopes of Narrowband Signals," Proc. IEEE 70, 1982, pp. 692-699.

21. Hadim, N., "Signal Estimation from Short-Time Spectral Magnitude," Ph.D. Thesis, MIT, 1982 